\title{
Discretização de modelos com difusão e retardo em domínios unidimensionais
}

\author{
Maria do Carmo Pacheco de Toledo Costa
}

TESE APRESENTADA

$\mathrm{AO}$

INSTITUTO DE MATEMÁTICA E ESTATÍSTICA

DA

UNIVERSIDADE DE SÃO PAULO

PARA

OBTENÇÃO DO TÍTULO DE DOUTORA

EM

CIÊNCIAS - ÁREA: MATEMÁTICA APLICADA

Área de concentração: Matemática Aplicada

Orientador: Prof. Dr. Sergio Muniz Oliva Filho

- Durante a elaboração deste trabalho a autora recebeu auxílio financeiro da CAPES -

São Paulo

2004 


\section{Discretização de modelos com difusão e retardo em domínios unidimensionais}

Este exemplar corresponde à redação final da tese de doutorado devidamente corrigida e defendida por Maria do Carmo Pacheco de Toledo Costa e aprovada pela comissão julgadora.

São Paulo, 14 junho de 2004.

Banca examinadora:

- Prof. Dr. Sergio Muniz Oliva Filho (Presidente) - IME - USP

- Prof. Dr. Antônio Luiz Pereira - IME - USP

- Prof. Dr. Plácido Zoega Táboas - ICMC - USP

- Prof. Dr. Alexandre Nolasco de Carvalho - ICMC - USP

- Profa. Dra. Simone Mazini Bruschi - IGCE - UNESP 
Ao Heitor, por estes anos de companheirismo, alegria e amor. 


\section{Agradeço}

ao Prof. Sergio, meu orientador, pela sensibilidade e dedicação com que me auxiliou na elaboração deste trabalho e que foram sempre um grande estímulo;

aos meus pais, amigos muito especiais. Adoro viver junto a vocês!

à Ana Cristina, minha irmã e amiga, pela presença;

à tia Neti, pelo carinho;

aos professores e funcionários do Departamento de Ciências Exatas

da Universidade Federal de Lavras, pelo ambiente acolhedor;

ao Prof. Antônio Luiz, que me ajudou discutindo parte deste trabalho;

à Alice, que contribuiu para que esta jornada fosse mais leve e agradável;

à Olga, pela ajuda com o uso do Linux. 


\section{Resumo}

Neste trabalho encontramos um sistema de dimensão finita cuja dinâmica aproxima, com a precisão que quisermos, a dinâmica assintótica da equação de reação e difusão com retardo finito

$$
\begin{cases}u_{t}(x, t)=a u_{x x}(x, t)+u(x, t)(1-u(x, t-r)), & \text { se } 0<x<1, t>0 \\ u_{x}(0, t)=u_{x}(1, t)=0, & \text { se } t \geq 0, \\ u(x, t)=\varphi(x, t)>0, & \text { se } 0<x<1, t \in[-r, 0]\end{cases}
$$

que tem sido usada como modelo de crescimento populacional com densidade da população $u$. O sistema de dimensão finita é obtido através de uma discretização do retardo e de uma discretização espacial. Ao fazermos a discretização do retardo estimamos, diretamente, a proximidade das soluções do problema contínuo e discretizado. Com a discretização espacial obtemos, em cada caso, uma variedade invariante exponencialmente atratora e comparamos os campos vetoriais, dos problemas contínuo e discretizado, sobre a variedade invariante. 


\section{Abstract}

In this work we consider the reaction-diffusion equation with finite

delay

$$
\begin{cases}u_{t}(x, t)=a u_{x x}(x, t)+u(x, t)(1-u(x, t-r)), & \text { se } 0<x<1, t>0 \\ u_{x}(0, t)=u_{x}(1, t)=0, & \text { se } t \geq 0 \\ u(x, t)=\varphi(x, t)>0, & \text { se } 0<x<1, t \in[-r, 0]\end{cases}
$$

which has been used as a model for the evolution of a population with density distribution $u$. Since the continuous problem is infinite dimensional, the first task is to reduce it to a problem on a finite dimensional space. That is achieved by a discretization in time and in space. Finally, we prove that the discretized problem contain the same asymptotic dynamics as the continuous problem. 


\section{Sumário}

Introdução

1 Equações diferenciais funcionais abstratas $\quad 8$

1.1 Existência de soluções para problemas com retardo finito . . 10

1.2 Existência de soluções para problemas com retardo infinito . . 36

2 Problema contínuo $\quad 46$

2.1 Existência e unicidade de soluções . . . . . . . . . . . . . . 47

2.2 Atrator global . . . . . . . . . . . . . . . 49

3 Problema discretizado $\quad 55$

3.1 Discretização do retardo . . . . . . . . . . 56

3.2 Proximidade das soluções do problema contínuo e discretizado 58

3.3 Discretização espacial . . . . . . . . . . . . . . . 74

$\begin{array}{ll}\text { Referências Bibliográficas } & 96\end{array}$ 


\section{Introdução}

Consideremos uma população formada somente por uma espécie, distribuída uniformemente em um meio ambiente isolado, e seja $u(t)$ a densidade da população em um instante de tempo t. O mais simples dos modelos de crescimento populacional considera que a taxa de crescimento é diretamente proporcional ao tamanho da população

$$
\frac{d u(t)}{d t}=b u(t)
$$

sendo $b$ uma constante positiva chamada taxa de crescimento da população [21].

Mas a solução desta equação diferencial resulta em um crescimento exponencial da população e, como o crescimento de uma população deve ser limitado, em função da disponibilidade de recursos, uma extensão natural deste modelo, que sana essa deficiência, é assumir que a taxa de crescimento é uma função da população

$$
\frac{d u(t)}{d t}=b u(t) f(u(t))
$$

Nesta extensão, deseja-se que quando o tamanho da população $u(t)$ for pequeno haja recursos suficientes, de modo que a taxa de crescimento será independente dos recursos e assim, aproximadamente proporcional à população. Entretanto, à medida que a população aumenta, os recursos disponíveis por indivíduo diminuirão e produzirão um efeito inibitório no crescimento 
populacional e portanto $f(u(t))$ deverá diminuir. Uma função que satisfaz estas condições, e é muito utilizada para estes modelos, é $f(u(t))=1-\frac{u(t)}{K}$, com $K$ sendo a capacidade de sustentação do meio ambiente, a qual é determinada por fatores como oferta de alimentos, espaço, predação. Temos assim a chamada equação logística [1]

$$
\frac{d u(t)}{d t}=b u(t)\left(1-\frac{u(t)}{K}\right)
$$

Na equação acima, a razão de crescimento da população depende da população em um dado instante de tempo $t$, mas na maioria das situações reais os efeitos regulatórios operam com alguma defasagem temporal $r$, que pode ser originado por gestação, período de maturação (devido ao estágio larval em populações de insetos). Como, então, modelar uma dinâmica de população incorporando esta defasagem? A resposta a esta questão é uma extensão natural da equação logística e é proposta por Hutchinson [18]

$$
\frac{d u(t)}{d t}=b u(t)\left(1-\frac{u(t-r)}{K}\right)
$$

Continuando com a idéia de tornar o modelo mais realista, observamos que os efeitos regulatórios não devem depender da população de um tempo defasado exatamente de $r$, mas sim de uma média das populações passadas, isto é, o termo $\frac{u(t-r)}{K}$ pode ser melhor modelado com $\int_{-\infty}^{t} \frac{u(s) Q(t-s)}{K} d s$, sendo $Q:[0, \infty) \rightarrow[0, \infty)$ uma aplicação com um impulso em $r$, que decai exponencialmente em $+\infty$ e com $\int_{0}^{\infty} Q(s) d s=1$; esta aplicação $Q$ descreve a influência do efeito do retardo $s$ unidades de tempo depois de $t$. Assim, a equação torna-se

$$
\frac{d u(t)}{d t}=b u(t)\left(1-\frac{1}{K} \int_{-\infty}^{t} u(s) Q(t-s) d s\right)
$$


Equações como (0.1) e (0.2) já foram muito estudadas, por exemplo, Travis e Webb $[27,28]$ mostraram existência e unicidade de soluções e propriedades de estabilidade da equação (0.1) e resultados similares, para a equação (0.2), podem ser encontrados no trabalho de Hale e Kato [9], no qual também introduziram a definição de espaço de fase para equações com retardo infinito. É interessante notar que podemos encontrar uma seqüência de funções $\left\{Q_{n}(t)\right\}$ que, em algum sentido, converge à função $\delta$ de Dirac e neste caso o modelo (0.2) converge ao modelo (0.1), ou seja, o modelo com retardo finito (modelo (0.1)) pode ser visto como um caso limite para o modelo com retardo infinito (modelo $(0.2)$ ), veja em particular $[7,15,16]$. Construiremos uma seqüência para a qual a não linearidade converge uniformemente sobre conjuntos eqüicontínuos; para maiores detalhes veja seção 3.2.

Em muitos sistemas ecológicos há um movimento das espécies (dispersão e migração) de um local a outro além da evolução temporal. Esta difusão espacial provém da tendência das espécies migrarem para regiões com baixa densidade populacional, pois em regiões com alta densidade populacional a comida ficará escassa [30]. Do ponto de vista matemático esta dependência espacial do meio ambiente foi completamente desprezada nos modelos anteriores.

Os modelos matemáticos que melhor incorporam estas características são as chamadas equações diferenciais funcionais parciais. Consideraremos, como protótipo de estudo, um modelo cuja população seja constituída por uma única espécie que habita um espaço de uma única dimensão e temos, como exemplo, a equação de reação e difusão conhecida, também, como 
equação de Hutchinson

$$
u_{t}(x, t)=a u_{x x}(x, t)+b u(x, t)\left(1-\frac{u(x, t-r)}{K}\right), t>0
$$

com $a$ e $b$ constantes positivas e $r$ a defasagem temporal.

Se o meio ambiente tem comprimento finito, por exemplo $L$, alguma condição na fronteira deve ser imposta. As condições de fronteira de Dirichlet

$$
u(0, t)=u(L, t)=0
$$

que representam a situação onde o meio ambiente é circundado por regiões totalmente desfavoráveis, ou de Neumann

$$
u_{x}(0, t)=u_{x}(L, t)=0
$$

que descrevem a situação em que a população não pode cruzar a fronteira de seu meio ambiente, são as mais usuais. Tais modelos, com retardamento e difusão, já foram muito estudados, por exemplo, Oliveira [23] mostrou existência, unicidade e dependência contínua de tais modelos, Luckhaus [19] mostrou limitação das soluções em domínios unidimensionais e Friesecke [6] a não limitação das soluções em domínios de dimensões maiores, Sergio e Cònsul [22] mostraram propriedades de sincronização e Ruan e Wu [25] estudaram vários resultados sobre existência e comportamento assintótico de soluções para equações de reação e difusão com retardo infinito; veja também $[3,4,14,26,29,31]$.

Assim, neste trabalho, vamos considerar somente a equação protótipo$$
\begin{cases}u_{t}(x, t)=a u_{x x}(x, t)+u(x, t)(1-u(x, t-r)), & \text { se } 0<x<1, t>0, \\ u_{x}(0, t)=u_{x}(1, t)=0, & \text { se } t \geq 0, \\ u(x, t)=\varphi(x, t)>0, & \text { se } 0<x<1, t \in[-r, 0]\end{cases}
$$ 
porém, os resultados podem ser facilmente generalizados para outras situações.

Observemos que a condição $u(x, t)=\varphi(x, t)$, se $0<x<1 \mathrm{e}$ $t \in[-r, 0]$, é a condição inicial para o problema, e será denotada, por simplicidade, por $u_{0}=\varphi$ e a fim de ser um problema plausível do ponto de vista biológico, iremos considerar a condição inicial positiva, ressaltando que sabemos que as soluções positivas formam um conjunto invariante [31].

Nosso objetivo, neste trabalho, é encontrar um sistema de dimensão finita cuja dinâmica possa aproximar, com a precisão que quisermos, a dinâmica assintótica da equação (0.3). Em outras palavras, construiremos uma nova forma de discretizar a equação (0.3) e mostrar a aproximação da dinâmica em uma vizinhança do atrator global. Ressaltamos que tal discretização deve lidar com duas "dinâmicas" de dimensão infinita, a difusão espacial e o retardamento temporal.

A princípio, usamos teoria de semigrupo para mostrar existência e unicidade de soluções do problema dado. Mostramos também que estas soluções geram um semigrupo o qual possui atrator global, e estes resultados aparecem no capítulo 2 desta tese.

A seguir, com o propósito de reduzirmos o estudo da dinâmica assintótica do problema dado em espaço de dimensão infinita ao estudo da dinâmica assintótica do problema dado em espaço de dimensão finita, faremos a discretização em duas etapas, primeiro apresentando um sistema, ainda de dimensão infinita, resultado da discretização do retardo, mais precisamente do intervalo $[-r, 0]$. Esta etapa inspira-se nas discretizações de equações retardadas utilizadas em Gedeon e Hines [7] e Hines [15]. Na segunda etapa, apresentamos um sistema de dimensão finita, resultado da discretização 
espacial.

A primeira discretização que introduzimos transforma o problema dado no estudo de um sistema formado por uma equação diferencial parcial e um sistema de equações diferenciais ordinárias. Isto é realizado pela discretização do retardo, transformando o intervalo de influência do retardo, ou seja, $[-r, 0]$, em $n+1$ valores discretos com um espaçamento constante $\Delta t=\frac{r}{n}$, assim obtemos o sistema

$$
\left\{\begin{array}{l}
\left(U_{0}(x, t)\right)_{t}=a\left(U_{0}(x, t)\right)_{x x}+U_{0}(x, t)\left(1-U_{n}(x, t)\right) \\
\left(U_{1}(x, t)\right)_{t}=\frac{n}{r}\left(U_{0}(x, t)-U_{1}(x, t)\right) \\
\vdots \\
\left(U_{n}(x, t)\right)_{t}=\frac{n}{r}\left(U_{n-1}(x, t)-U_{n}(x, t)\right)
\end{array}\right.
$$

como será discutido na seção 3.1 .

Ao fazermos a discretização do retardo estimamos, diretamente, a proximidade das soluçôes do problema contínuo e discretizado. O principal objetivo da seção 3.2 será demonstrar que, dada uma solução $u$ de $(0.3)$ e $U_{0}$ de $(0.4)$, com condições iniciais compatíveis, temos $\left\|u(t)-U_{0}(t)\right\|_{H^{1}} \rightarrow 0$ uniformemente em $t$ quando $n \rightarrow \infty$. Para isto, consideramos um problema com retardo infinito e mostramos que suas soluções convergem às soluções do problema dado e a seguir que o problema com retardo infinito e o problema discretizado são equivalentes.

Para a próxima etapa seguiremos os resultados de Bruschi, Carvalho e Ruas-Filho [2] para obtermos a discretização espacial, que será apresentada na seção 3.3. Obtemos, em cada caso, uma variedade invariante exponencialmente atratora e comparamos os campos vetoriais, do problema contínuo e discretizado, sobre a variedade invariante. Na realidade, mostramos a convergência uniforme dos autovalores e autofunções, mas para isto é preciso que a dimensão do problema discretizado exceda a dimensão da variedade 
sobre a qual o problema contínuo está sendo considerado.

Julgamos interessante comentar que não é possível fazermos inicialmente a discretização espacial e depois a discretização do retardo, pois para o problema dado não temos variedade invariante de dimensão finita e assim não conseguimos um controle na convergência dos autovalores e autofunções. 


\section{Capítulo 1}

\section{Equações diferenciais funcionais abstratas}

Neste capítulo apresentamos alguns resultados abstratos sobre existência de soluções de equações diferenciais funcionais com retardo finito (seção 1.1) e com retardo infinito (seção 1.2). Além disso, mostramos que, em alguns casos, as soluções de equações com retardo finito definem um semigrupo fortemente contínuo e compacto.

Vamos agora lembrar algumas definições que serão utilizadas adiante $[13,24]$.

Definição 1.1 Um operador linear $A$ definido em um espaço de Banach $X$ é dito ser um operador setorial se é fechado, densamente definido e se existem $\theta \in\left(0, \frac{\pi}{2}\right)$ e constantes $M \geq 1$ e $a \in \mathbb{R}$ tal que o setor

$$
S_{a, \theta}=\{z ; \theta \leq|\arg (z-a)| \leq \pi, z \neq a\}
$$

está contido no conjunto resolvente de $A$ e

$$
\left\|(z I-A)^{-1}\right\| \leq \frac{M}{|z-a|}
$$

para todo $z \in S_{a, \theta}$. 
Definição 1.2 Um semigrupo analítico em um espaço de Banach $X$ é uma familia de operadores lineares contínuos sobre $X,\{T(t)\}_{t \geq 0}$, satisfazendo

(i) $T(0)=I, T(t) T(s)=T(t+s)$ para $t \geq 0, s \geq 0$;

(ii) $T(t) x \rightarrow x$ quando $t \rightarrow 0_{+}$, para cada $x \in X$;

(iii) $t \rightarrow T(t) x$ é analítica sobre $0<t<\infty$ para cada $x \in X$.

O gerador infinitesimal deste semigrupo é o operador linear definido por $L: D(L) \subset X \rightarrow X$ com $D(L)=\left\{x \in X ; \lim _{t \rightarrow 0_{+}} \frac{T(t) x-x}{t}\right.$ existe $\} e$ $L(x)=\lim _{t \rightarrow 0_{+}} \frac{T(t) x-x}{t}$. Usualmente escrevemos $T(t)=e^{L t}$.

Definição 1.3 Seja A um operador setorial definido em um espaço de Banach $X$.

(i) Se Re $\sigma(A)>0$, para todo $\alpha>0$ definimos

$$
A^{-\alpha}=\frac{1}{\Gamma(\alpha)} \int_{0}^{\infty} t^{\alpha-1} e^{-A t} d t
$$

sendo $\Gamma(\alpha)=\int_{0}^{\infty} t^{\alpha-1} e^{-t} d t$

Definimos também $A^{\alpha}=\left(A^{-\alpha}\right)^{-1} \operatorname{com} D\left(A^{\alpha}\right)=\operatorname{Im}\left(A^{-\alpha}\right)$ e $A^{0}=I_{X}$.

(ii) Para todo $\alpha \geq 0$ definimos

$$
X^{\alpha}=D\left(A_{1}^{\alpha}\right)
$$

com a norma do gráfico, ou seja, $\|x\|_{X^{\alpha}}=\left\|A_{1}^{\alpha} x\right\|, x \in X^{\alpha}$, sendo $A_{1}=A+a I$ com a escolhido de forma que $\operatorname{Re} \sigma\left(A_{1}\right)>0$.

Cabe observar que diferentes escolhas de a definem normas equivalentes. 
Para estudarmos equações diferenciais funcionais abstratas vamos considerar $X$ um espaço de Banach e $A: D(A) \subset X \rightarrow X$ um operador setorial com $-A$ o gerador infinitesimal de um semigrupo analítico $\left\{e^{-A t}\right\}_{t \geq 0}$ satisfazendo $\left\|e^{-A t}\right\|_{\mathcal{L}(X)} \leq M$ para todo $t \geq 0$ e $M \geq 1$ uma constante. Observemos que, neste caso, podemos considerar os espaços $X^{\alpha}, 0 \leq \alpha<1$, associados às potências fracionárias $A^{\alpha}$ de $A[13,24]$.

\subsection{Existência de soluções para problemas com retardo finito}

As demonstrações apresentadas nesta seção são baseadas no trabalho de Oliveira [23].

Sejam $r \geq 0$ um número real dado e $C\left([-r, 0], X^{\alpha}\right)$ o espaço de Banach das funçôes contínuas $\varphi:[-r, 0] \rightarrow X^{\alpha}$ com a norma do supremo. E para todo número real $a>0, t \in[0, a]$ e toda função contínua $u:[-r, a) \rightarrow X^{\alpha}$, seja $u_{t}$ a função em $C\left([-r, 0], X^{\alpha}\right)$ definida por $u_{t}(\theta)=u(t+\theta)$.

Apresentamos, a seguir, detalhes quanto à existência, unicidade e dependência contínua de soluções da equação

$$
\dot{u}(t)+A u(t)=F\left(t, u_{t}\right), \text { para todo } t>0,
$$

com $F: \mathbb{R} \times C\left([-r, 0], X^{\alpha}\right) \rightarrow X$ contínua e localmente Lipschitz contínua em seu segundo argumento.

Definição 1.4 Uma função contínua $u:[-r, T) \rightarrow X^{\alpha}, \operatorname{com} T>r$, é solução de (1.1) com condição inicial $u_{0}=\varphi \in C\left([-r, 0], X^{\alpha}\right)$ se 
(i) $u(t)=\varphi(t)$, para todo $-r \leq t \leq 0$;

(ii) $u$ é solução da equação integral

$$
u(t)=e^{-A t} \varphi(0)+\int_{0}^{t} e^{-A(t-s)} F\left(s, u_{s}\right) d s,
$$

para todo $0<t \leq r$

(iii) $u$ é de classe $C^{1}\left((r, T), X^{\alpha}\right), u(t) \in D(A)$ e $\dot{u}(t)+A u(t)=F\left(t, u_{t}\right)$, para todo $r<t<T$.

Julgamos importante observar que a hipótese $T>r$ na definição acima não é problema para a equação que abordaremos, pois, como veremos, hipóteses sobre a não linearidade implicarão na existência global de soluções.

Para mostrarmos existência de soluções da equação (1.1)

$$
\dot{u}(t)+A u(t)=F\left(t, u_{t}\right), \text { para todo } t>0
$$

com condição inicial $u_{0}=\varphi \in C\left([-r, 0], X^{\alpha}\right)$ mostramos, a princípio, existência de soluções da equação integral

$$
u(t)=e^{-A t} \varphi(0)+\int_{0}^{t} e^{-A(t-s)} F\left(s, u_{s}\right) d s,
$$

para todo $t>0$ com $u(t)=\varphi(t)$ se $-r \leq t \leq 0$ e em seguida, com hipóteses adicionais sobre $F$, mostramos que uma solução de (1.2) é solução de (1.1) $\operatorname{com} u_{0}=\varphi \in C\left([-r, 0], X^{\alpha}\right)$.

Iniciamos com um resultado bem conhecido na teoria de semigrupo que se faz necessário às demonstrações a seguir.

Teorema 1.5 Seja $A: D(A) \subset X \rightarrow X$ um operador setorial satisfazendo $\operatorname{Re} \sigma(A)>\lambda>0$, então 
(i) se $\alpha \geq 0$ e $x \in X,\left\|A^{\alpha} e^{-A t} x\right\|_{X} \leq N_{\alpha} t^{-\alpha} e^{-\lambda t}\|x\|_{X}$, para $t>0$

(ii) se $0<\alpha \leq 1$ e $x \in D\left(A^{\alpha}\right),\left\|\left(e^{-A t}-I\right) x\right\|_{X} \leq \frac{1}{\alpha} N_{1-\alpha} t^{\alpha}\|x\|_{X^{\alpha}}$, para $t \geq 0$

A demonstração deste teorema pode ser encontrada em Henry [13] ou Pazy [24].

Com o propósito de simplificarmos a notação neste trabalho o espaço $C\left([-r, 0], X^{\alpha}\right)$ será, algumas vezes, denotado simplesmente por $C_{\alpha}$.

Teorema 1.6 Seja $\left.F: \mathbb{R} \times C([-r, 0]), X^{\alpha}\right) \rightarrow X$ contínua e localmente Lipschitz contínua em seu segundo argumento. Dado $(\sigma, \varphi) \in \mathbb{R} \times C\left([-r, 0], X^{\alpha}\right)$ existem um número real $\rho=\rho(\sigma, \varphi)>0$ e uma única função contínua $u:[\sigma-r, \sigma+\rho] \rightarrow X^{\alpha}$, tal que $u_{\sigma}=\varphi e$

$$
u(t)=e^{-A(t-\sigma)} \varphi(0)+\int_{\sigma}^{t} e^{-A(t-s)} F\left(s, u_{s}\right) d s
$$

para todo $\sigma \leq t \leq \sigma+\rho$.

\section{Demonstração}

Como $F$ é localmente Lipschitz contínua em seu segundo argumento existem $\delta>0, \tau>0$ e constantes positivas $L$ e $D$ tais que se $|t-\sigma| \leq \tau$, $\left\|\varphi_{1}-\varphi\right\|_{C_{\alpha}} \leq \delta \mathrm{e}\left\|\varphi_{2}-\varphi\right\|_{C_{\alpha}} \leq \delta$ então

$$
\left\|F\left(t, \varphi_{1}\right)-F\left(t, \varphi_{2}\right)\right\|_{X} \leq L\left\|\varphi_{1}-\varphi_{2}\right\|_{C_{\alpha}}
$$

e

$$
\left\|F\left(t, \varphi_{1}\right)\right\|_{X} \leq D .
$$


Consideremos a função $w:[\sigma-r, \sigma+\tau] \rightarrow X^{\alpha}$ definida por

$$
w(t)= \begin{cases}e^{-A(t-\sigma)} \varphi(0), & \text { se } \sigma<t \leq \sigma+\tau \\ \varphi(t-\sigma), & \text { se } \sigma-r \leq t \leq \sigma\end{cases}
$$

e como $w$ é uniformemente contínua existe $\varepsilon>0$ tal que $\|w(t)-w(s)\|_{X^{\alpha}} \leq \frac{\delta}{2}$ se $t, s \in[\sigma-r, \sigma+\tau]$ e $|t-s| \leq \varepsilon$.

Seja $\rho=\min \{\tau, \varepsilon\} \operatorname{com} \frac{N_{\alpha} D \rho^{1-\alpha}}{1-\alpha} \leq \frac{\delta}{2}$ e $\frac{N_{\alpha} L \rho^{1-\alpha}}{1-\alpha} \leq \frac{1}{2}$.

Então, a restrição de $w$ ao intervalo $[\sigma-r, \sigma+\rho]$, que ainda será indicada por $w$, satisfaz

$$
\|w(t+\theta)-w(s+\theta)\|_{X^{\alpha}} \leq \frac{\delta}{2}
$$

para $t, s \in[\sigma, \sigma+\rho] \operatorname{com}|t-s| \leq \varepsilon$ e $-r \leq \theta \leq 0$.

Em particular, para $s=\sigma$ temos

$$
\begin{aligned}
\|w(t+\theta)-w(\sigma+\theta)\|_{X^{\alpha}} & =\|w(t+\theta)-\varphi(\theta)\|_{X^{\alpha}} \\
& \leq \frac{\delta}{2}
\end{aligned}
$$

para $t \in[\sigma, \sigma+\rho] \mathrm{e}-r \leq \theta \leq 0$

Assim,

$$
\left\|e^{-A(t+\theta-\sigma)} \varphi(0)-\varphi(\theta)\right\|_{X^{\alpha}} \leq \frac{\delta}{2}, \quad \text { se } \quad \sigma<t+\theta \leq \sigma+\rho
$$

e

$$
\|\varphi(t+\theta-\sigma)-\varphi(\theta)\|_{X^{\alpha}} \leq \frac{\delta}{2}, \quad \text { se } \sigma-r \leq t+\theta \leq \sigma
$$

Consideremos o conjunto $S$ das funções $u:[\sigma-r, \sigma+\rho] \rightarrow X^{\alpha}$ contínuas, com $u_{\sigma}=\varphi,\left\|u_{t}-\varphi\right\|_{c_{\alpha}} \leq \delta$ para $\sigma \leq t \leq \sigma+\rho$ e com norma

$$
\|u-v\|_{S}=\sup _{\sigma \leq t \leq \sigma+\rho}\left\|u_{t}-v_{t}\right\|_{C_{\alpha}}
$$


E definimos $G: S \rightarrow C\left([\sigma-r, \sigma+\rho], X^{\alpha}\right)$ por

$(G u)(t)= \begin{cases}e^{-A(t-\sigma)} \varphi(0)+\int_{\sigma}^{t} e^{-A(t-s)} F\left(s, u_{s}\right) d s, & \text { se } \sigma<t \leq \sigma+\rho \\ \varphi(t-\sigma), & \text { se } \sigma-r \leq t \leq \sigma .\end{cases}$

Devemos mostrar que $G$ tem um único ponto fixo em $S$ e para isto, usando o teorema do ponto fixo de Banach, basta mostrarmos que $G(S) \subset S$ e $G$ é uma contração, observando que $S$ é fechado.

Seja $u \in S$. Então, para $\sigma-r \leq t \leq \sigma$ temos que $(G u)(t)=\varphi(t-\sigma)$ e portanto $(G u)_{\sigma}(\theta)=\varphi(\theta)$ para todo $-r \leq \theta \leq 0$.

E para $\sigma \leq t \leq \sigma+\rho$ temos $(G u)_{t}(\theta)-\varphi(\theta)= \begin{cases}e^{-A(t+\theta-\sigma)} \varphi(0)-\varphi(\theta) & \\ \quad+\int_{\sigma}^{t+\theta} e^{-A(t+\theta-s)} F\left(s, u_{s}\right) d s, & \text { se } \sigma<t+\theta \leq \sigma+\rho \\ \varphi(t+\theta-\sigma)-\varphi(\theta), & \text { se } \sigma-r \leq t+\theta \leq \sigma\end{cases}$ para todo $-r \leq \theta \leq 0$.

Portanto, de (1.4) e (1.5), segue $\left\|(G u)_{t}(\theta)-\varphi(\theta)\right\|_{X^{\alpha}} \leq \begin{cases}\frac{\delta}{2}+\frac{N_{\alpha} D \rho^{1-\alpha}}{1-\alpha} \leq \delta, & \text { se } \sigma<t+\theta \leq \sigma+\rho \\ \frac{\delta}{2}, & \text { se } \sigma-r \leq t+\theta \leq \sigma\end{cases}$ para todo $-r \leq \theta \leq 0$, o que resulta

$$
\left\|(G u)_{t}-\varphi\right\|_{C_{\alpha}} \leq \delta, \text { para todo } \sigma \leq t \leq \sigma+\rho
$$

A fim de provarmos que $G$ é uma contração consideremos $u, v \in S$, $-r \leq \theta \leq 0$ e $\sigma \leq t \leq \sigma+\rho$. Então, $(G u)_{t}(\theta)-(G v)_{t}(\theta)=\left\{\begin{array}{rr}\int_{\sigma}^{t+\theta} e^{-A(t+\theta-s)}\left[F\left(s, u_{s}\right)-F\left(s, v_{s}\right)\right] d s \\ \text { se } \sigma<t+\theta \leq \sigma+\rho \\ 0, & \text { se } \sigma-r \leq t+\theta \leq \sigma\end{array}\right.$ e portanto,

$$
\left\|(G u)_{t}(\theta)-(G v)_{t}(\theta)\right\|_{X^{\alpha}}
$$




$$
\begin{aligned}
& \leq N_{\alpha} L \sup _{\substack{\sigma \leq s \leq t+\theta \leq \sigma+\rho \\
1-\alpha}}\left\|u_{s}-v_{s}\right\|_{C_{\alpha}} \frac{(t+\theta-\sigma)^{1-\alpha}}{1-\alpha} \\
& \leq \frac{N_{\alpha} L \rho^{1-\alpha}}{1-\alpha}\|u-v\|_{S} \\
& \leq \frac{1}{2}\|u-v\|_{S} .
\end{aligned}
$$

Portanto,

$$
\left\|(G u)_{t}-(G v)_{t}\right\|_{C_{\alpha}} \leq \frac{1}{2}\|u-v\|_{S}
$$

para todo $\sigma \leq t \leq \sigma+\rho$ e assim,

$$
\|G u-G v\|_{S} \leq \frac{1}{2}\|u-v\|_{s}
$$

Como conseqüência do teorema do ponto fixo de Banach temos que $G$ tem um único ponto fixo em $S$ e assim segue o resultado desejado.

O lema a seguir nos garante unicidade global e existência de intervalo maximal de definição para solução da equação integral (1.3).

Lema 1.7 Sejam $u, v:[\sigma-r, \sigma+\beta] \rightarrow X^{\alpha}$, com $\sigma \in \mathbb{R}$ e $\beta>0$, soluções contínuas da equação integral (1.3)

$$
u(t)=e^{-A(t-\sigma)} \varphi(0)+\int_{\sigma}^{t} e^{-A(t-s)} F\left(s, u_{s}\right) d s
$$

para todo $\sigma \leq t \leq \sigma+\beta$ com $u_{\sigma}=v_{\sigma}=\varphi$. Então $u=v$ em $[\sigma-r, \sigma+\beta]$.

\section{Demonstração}

$$
\text { Seja } I=\{t \in[\sigma-r, \sigma+\beta] ; u(s)=v(s) \text { para } \sigma-r \leq s \leq t\} \mathrm{e}
$$
observemos que são de verificação imediata que $I$ é um conjunto não vazio, limitado superiormente e fechado. 
Consideremos $\gamma=\sup I ;$ como $I$ é fechado, $\gamma \in I$, isto é, $u(s)=v(s)$ para $\sigma-r \leq s \leq \gamma$, assim, do teorema 1.6, segue que $\gamma>\sigma$

Suponhamos agora que $\gamma<\sigma+\beta$ e consideremos $\psi=u_{\gamma}=v_{\gamma}$, $(\gamma, \psi) \in \mathbb{R} \times C\left([-r, 0], X^{\alpha}\right)$, condição inicial para solução da equação (1.3), e usando novamente o teorema 1.6 existe $\tau>0$, e podemos considerar $0<\tau<-\gamma+\sigma+\beta$, e uma única solução $u^{*}:[\gamma-r, \gamma+\tau] \rightarrow X^{\alpha}$ da equação (1.3).

Notemos também que $u$ e $v$ são soluções da equação (1.3) no intervalo $[\gamma-r, \gamma+\tau] \operatorname{com} u_{\gamma}=v_{\gamma}=\psi$ e pela unicidade da solução temos que $u(t)=v(t)=u^{*}(t)$ para todo $\gamma-r \leq t \leq \gamma+\tau$.

Assim, $\gamma+\tau \in I$, o que contradiz o fato de $\gamma=\sup I$.

E portanto, $\gamma=\sigma+\beta$.

Para cada $(\sigma, \varphi) \in \mathbb{R} \times C\left([-r, 0], X^{\alpha}\right)$ definimos

$$
\begin{aligned}
\rho^{*}(\sigma, \varphi)= & \sup \{\rho>\sigma ; \text { a equação integral (1.3) possui uma solução } \\
& \text { contínua em }[\sigma-r, \rho]\}
\end{aligned}
$$

e seja $u(\sigma, \varphi):\left[\sigma-r, \rho^{*}(\sigma, \varphi)\right) \rightarrow X^{\alpha}$ definida por $u(\sigma, \varphi)(t)=\varphi(t-\sigma)$, se $\sigma-r \leq t \leq \sigma$ e se $\sigma<t<\rho^{*}(\sigma, \varphi), u(\sigma, \varphi)(t)$ é igual ao valor da solução da equação integral (1.3), no instante $t$, com condição inicial $u_{\sigma}=\varphi$, definida em $[\sigma-r, \rho] \operatorname{com} t \leq \rho$.

Como conseqüência do lema acima temos que $u(\sigma, \varphi)$ é uma função contínua e bem definida no intervalo $\left[\sigma-r, \rho^{*}(\sigma, \varphi)\right)$ e é uma solução de (1.3), satisfazendo $u_{\sigma}=\varphi$, chamada solução maximal.

O intervalo de existência de uma solução maximal da equação integral (1.3) deve ser aberto à direita e o caso $\rho^{*}(\sigma, \varphi)=\infty$ não é excluído. 
A proposição 1.8 abaixo nos garante que a solução $u(\sigma, \varphi)$ da equação integral (1.3) depende continuamente do dado inicial $\varphi \in C\left([-r, 0], X^{\alpha}\right)$.

Proposição 1.8 Seja $u=u(\sigma, \varphi)$ a solução da equação integral (1.3)

$$
u(t)=e^{-A(t-\sigma)} \varphi(0)+\int_{\sigma}^{t} e^{-A(t-s)} F\left(s, u_{s}\right) d s
$$

definida em $[\sigma-r, \rho)$ para algum $\rho>\sigma$ e satisfazendo $u_{\sigma}=\varphi$ e seja $T$ um número real tal que $\sigma<T<\rho$. Então existe um número $\delta>0$ tal que qualquer solução $v=v(\sigma, \psi)$ da equação integral (1.3), com $v_{\sigma}=\psi e$ $\|\varphi-\psi\|_{C_{\alpha}}<\delta$, está definida pelo menos em $[\sigma-r, T]$. Além disso, para $t$ fixo, $\sigma \leq t \leq T$, a aplicação $\varphi \mapsto u_{t}(\sigma, \varphi)$ é contínua.

\section{Demonstração}

Lembremos que $\left\|e^{-A t}\right\|_{\mathcal{C}(X)} \leq M$ para todo $t \geq 0$, como observado anteriormente.

Como $F$ é localmente Lipschitz contínua em seu segundo argumento existe uma constante positiva $L$ tal que

$$
\left\|F\left(t, \varphi_{1}\right)-F\left(t, \varphi_{2}\right)\right\|_{X} \leq L\left\|\varphi_{1}-\varphi_{2}\right\|_{C_{\alpha}}
$$

para $\left(t, \varphi_{1}\right)$ e $\left(t, \varphi_{2}\right)$ em uma vizinhança $V$ da curva $\left\{\left(t, u_{t}\right) ; \sigma \leq t \leq T\right\}$.

Observemos também que existe $\tau>0$ tal que se $\psi \in C\left([-r, 0], X^{\alpha}\right)$ e $\left\|\psi-u_{t}\right\|_{c_{\alpha}} \leq \tau$ para algum $\sigma \leq t \leq T$, então $(t, \psi) \in V$.

Consideremos

$$
\begin{aligned}
k & =M \\
& +2 M N_{\alpha} L \frac{(T-\sigma)^{(1-\alpha q) / q}}{(1-\alpha q)^{1 / q}}(T-\sigma)^{1 / p} \\
& \cdot \exp \left\{2 N_{\alpha} L \frac{(T-\sigma)^{(1-\alpha q) / q}}{(1-\alpha q)^{1 / q}}(T-\sigma)^{1 / p}\right\}
\end{aligned}
$$


com $p$ e $q$ números reais positivos tais que $p$ é par, $\frac{1}{p}+\frac{1}{q}=1$ e $\alpha q<1$ e com $N_{\alpha}$ dado pelo teorema 1.5 .

Sejam $\delta=\frac{\tau}{k}, \psi \in C\left([-r, 0], X^{\alpha}\right)$ satisfazendo $\|\varphi-\psi\|_{C_{\alpha}}<\delta$ e $v=v(\sigma, \psi)$ solução da equação (1.3) com $v_{\sigma}=\psi$.

Devemos mostrar que $v$ está definida, pelo menos, em $[\sigma-r, T]$ e para isto basta mostrarmos que $\left(t, v_{t}\right) \in V$.

Observemos que para os valores de $\sigma \leq t \leq T$ para os quais a solução $v$ existe temos

$$
u_{t}(\theta)-v_{t}(\theta)=\left\{\begin{aligned}
e^{-A(t+\theta-\sigma)}[\varphi(0)-\psi(0)] & \\
+\int_{\sigma}^{t+\theta} e^{-A(t+\theta-s)}\left[F\left(s, u_{s}\right)-F\left(s, v_{s}\right)\right] d s & \text { se } \sigma<t+\theta \leq T \\
\varphi(t+\theta-\sigma)-\psi(t+\theta-\sigma), & \text { se } \sigma-r \leq t+\theta \leq \sigma,
\end{aligned}\right.
$$

para todo $-r \leq \theta \leq 0$

Assim, para os valores de $\sigma \leq t \leq T$ para os quais $\left(s, v_{s}\right) \in V$ com $\sigma \leq s \leq t$

$$
\left\|u_{t}(\theta)-v_{t}(\theta)\right\|_{X^{\alpha}} \leq\left\{\begin{aligned}
& M\|\varphi(0)-\psi(0)\|_{X^{\alpha}} \\
&+N_{\alpha} L\left(\int_{\sigma}^{t+\theta}(t+\theta-s)^{-\alpha q} d s\right)^{1 / q} \\
&\left(\int_{\sigma}^{t+\theta}\left\|u_{s}-v_{s}\right\|_{C_{\alpha}}^{p} d s\right)^{1 / p} \\
& \leq M\|\varphi-\psi\|_{C_{\alpha}} \\
&+N_{\alpha} L \frac{(T-\sigma)^{(1-\alpha q) / q}}{(1-\alpha q)^{1 / q}}\left(\int_{\sigma}^{t}\left\|u_{s}-v_{s}\right\|_{C_{\alpha}}^{p} d s\right)^{1 / p}, \\
&\|\varphi-\psi\|_{C_{\alpha}} \text { se } \sigma<t+\theta \leq T
\end{aligned}\right.
$$

para todo $-r \leq \theta \leq 0$.

Assim, temos que

$$
\begin{aligned}
& \left\|u_{t}-v_{t}\right\|_{C_{\alpha}} \\
& \quad \leq M\|\varphi-\psi\|_{C_{\alpha}}+N_{\alpha} L \frac{(T-\sigma)^{(1-\alpha q) / q}}{(1-\alpha q)^{1 / q}}\left(\int_{\sigma}^{t}\left\|u_{s}-v_{s}\right\|_{C_{\alpha}}^{p} d s\right)^{1 / p}
\end{aligned}
$$


para os valores de $\sigma \leq t \leq T$ para os quais $\left(s, v_{s}\right) \in V \operatorname{com} \sigma \leq s \leq t \mathrm{e}$ portanto,

$$
\begin{aligned}
& {\left[\left\|u_{t}-v_{t}\right\|_{C_{\alpha}}-M\|\varphi-\psi\|_{C_{\alpha}}\right]^{p}} \\
& \leq\left[N_{\alpha} L \frac{(T-\sigma)^{(1-\alpha q) / q}}{(1-\alpha q)^{1 / q}}\right]^{p} \\
& . \quad \int_{\sigma}^{t} 2^{p}\left\{\left[\left\|u_{s}-v_{s}\right\|_{C_{\alpha}}-M\|\varphi-\psi\|_{C_{\alpha}}\right]^{p}+M^{p}\|\varphi-\psi\|_{C_{\alpha}}^{p}\right\} d s \\
& \leq\left[2 N_{\alpha} L \frac{(T-\sigma)^{(1-\alpha q) / q}}{(1-\alpha q)^{1 / q}}\right]^{p}(T-\sigma) M^{p}\|\varphi-\psi\|_{C_{\alpha}}^{p} \\
& \quad+\left[2 N_{\alpha} L \frac{(T-\sigma)^{(1-\alpha q) / q}}{(1-\alpha q)^{1 / q}}\right]^{p} \int_{\sigma}^{t}\left[\left\|u_{s}-v_{s}\right\|_{C_{\alpha}}-M\|\varphi-\psi\|_{C_{\alpha}}\right]^{p} d s
\end{aligned}
$$

assim, da desigualdade de Gronwall, segue que

$$
\begin{aligned}
& {\left[\left\|u_{t}-v_{t}\right\|_{C_{\alpha}}-M\|\varphi-\psi\|_{C_{\alpha}}\right]^{p}} \\
& \leq\left[2 N_{\alpha} L \frac{(T-\sigma)^{(1-\alpha q) / q}}{(1-\alpha q)^{1 / q}}\right]^{p}(T-\sigma) M^{p}\|\varphi-\psi\|_{C_{\alpha}}^{p} \\
& \cdot \exp \left\{\left[2 N_{\alpha} L \frac{(T-\sigma)^{(1-\alpha q) / q}}{(1-\alpha q)^{1 / q}}\right]^{p}(T-\sigma)\right\},
\end{aligned}
$$

o que implica,

$$
\begin{aligned}
\left\|u_{t}-v_{t}\right\|_{C_{\alpha}} & \leq M\|\varphi-\psi\|_{C_{\alpha}} \\
& +2 N_{\alpha} L \frac{(T-\sigma)^{(1-\alpha q) / q}}{(1-\alpha q)^{1 / q}}(T-\sigma)^{1 / p} M\|\varphi-\psi\|_{C_{\alpha}} \\
& \cdot \exp \left\{2 N_{\alpha} L \frac{(T-\sigma)^{(1-\alpha q) / q}}{(1-\alpha q)^{1 / q}}(T-\sigma)^{1 / p}\right\} \\
& \leq k\|\varphi-\psi\|_{C_{\alpha}},
\end{aligned}
$$

para os valores de $\sigma \leq t \leq T$ para os quais $\left(s, v_{s}\right) \in V$ com $\sigma \leq s \leq t$.

Como $\|\varphi-\psi\|_{C_{\alpha}}<\delta=\frac{\tau}{k}$, temos que $\left\|u_{t}-v_{t}\right\|_{C_{\alpha}} \leq \tau$, para todo $\sigma \leq t \leq T$, o que resulta $\left(t, v_{t}\right) \in V$ e assim segue o resultado desejado.

Para concluir, a continuidade da aplicação $\varphi \mapsto u_{t}(\sigma, \varphi)$, para $t$ fixo, 
$\sigma \leq t \leq T$, segue diretamente da estimativa $\left\|u_{t}-v_{t}\right\|_{C_{\alpha}} \leq k\|\varphi-\psi\|_{C_{\alpha}}$.

Os resultados a seguir nos permitem concluir que, em alguns casos, é possível termos existência global de soluções da equação integral (1.3).

Lema $1.9 \operatorname{Sejam}(\sigma, \varphi) \in \mathbb{R} \times C\left([-r, 0], X^{\alpha}\right)$ e $u:\left[\sigma-r, \rho^{*}\right) \rightarrow X^{\alpha} u m a$ solução maximal da equação integral (1.3)

$$
u(t)=e^{-A(t-\sigma)} \varphi(0)+\int_{\sigma}^{t} e^{-A(t-s)} F\left(s, u_{s}\right) d s
$$

satisfazendo $u_{\sigma}=\varphi$. Se $\rho^{*}<\infty$ então $\lim _{t \rightarrow \rho_{-}^{*}} \sup \frac{\left\|F\left(t, u_{t}\right)\right\|_{X}}{1+\left\|u_{t}\right\|_{C_{\alpha}}}=\infty$.

\section{Demonstração}

Convém observar que usamos, nesta demonstração, o teorema 1.5.

Suponhamos, por absurdo, que $\lim _{t \rightarrow \rho_{-}^{*}} \sup \frac{\left\|F\left(t, u_{t}\right)\right\|_{X}}{1+\left\|u_{t}\right\|_{C_{\alpha}}}<\infty$. Então existe uma constante positiva $D$ tal que $\left\|F\left(t, u_{t}\right)\right\|_{X} \leq D\left(1+\left\|u_{t}\right\|_{C_{\alpha}}\right)$ para todo $\sigma \leq t<\rho^{*}$

Mostremos, a princípio, que se $\sigma \leq t<\rho^{*}$ então $\left\|u_{t}\right\|_{C_{\alpha}}$ é limitada.

$$
\text { Considerando }-r \leq \theta \leq 0 \text { temos }
$$

$$
u_{t}(\theta)= \begin{cases}e^{-A(t+\theta-\sigma)} \varphi(0)+\int_{\sigma}^{t+\theta} e^{-A(t+\theta-s)} & F\left(s, u_{s}\right) d s, \\ & \text { se } \sigma<t+\theta<\rho^{*} \\ \varphi(t+\theta-\sigma), & \text { se } \sigma-r \leq t+\theta \leq \sigma\end{cases}
$$

e portanto,

$$
\left\|u_{t}(\theta)\right\|_{X^{\alpha}} \leq\left\{\begin{array}{c}
M\|\varphi(0)\|_{X^{\alpha}}+\int_{\sigma}^{t+\theta} N_{\alpha}(t+\theta-s)^{-\alpha} D\left(1+\left\|u_{s}\right\|_{C_{\alpha}}\right) d s \\
\leq M\|\varphi\|_{C_{\alpha}}+N_{\alpha} D \frac{(t+\theta-\sigma)^{1-\alpha}}{1-\alpha} \\
+N_{\alpha} D\left(\int_{\sigma}^{t+\theta}(t+\theta-s)^{-\alpha q} d s\right)^{1 / q}\left(\int_{\sigma}^{t+\theta}\left\|u_{s}\right\|_{C_{\alpha}}^{p} d s\right)^{1 / p} \\
\leq M\|\varphi\|_{C_{\alpha}}+N_{\alpha} D \frac{\left(\rho^{*}-\sigma\right)^{1-\alpha}}{1-\alpha} \\
+N_{\alpha} D \frac{\left(\rho^{*}-\sigma\right)^{(1-\alpha q) / q}}{(1-\alpha q)^{1 / q}}\left(\int_{\sigma}^{t}\left\|u_{s}\right\|_{C_{\alpha}}^{p} d s\right)^{1 / p}, \\
\text { se } \sigma<t+\theta<\rho^{*} \\
\|\varphi\|_{C_{\alpha}}, \quad \text { se } \sigma-r \leq t+\theta \leq \sigma,
\end{array}\right.
$$


com $p$ e $q$ números reais positivos tais que $p$ é par, $\frac{1}{p}+\frac{1}{q}=1$ e $\alpha q<1$.

Assim,

$$
\begin{aligned}
\left\|u_{t}\right\|_{C_{\alpha}} & \leq M\|\varphi\|_{C_{\alpha}}+N_{\alpha} D \frac{\left(\rho^{*}-\sigma\right)^{1-\alpha}}{1-\alpha} \\
& +N_{\alpha} D \frac{\left(\rho^{*}-\sigma\right)^{(1-\alpha q) / q}}{(1-\alpha q)^{1 / q}}\left(\int_{\sigma}^{t}\left\|u_{s}\right\|_{C_{\alpha}}^{p} d s\right)^{1 / p}
\end{aligned}
$$

para todo $\sigma \leq t<\rho^{*}$.

Temos assim que

$$
\begin{aligned}
& {\left[\left\|u_{t}\right\|_{C_{\alpha}}-M\|\varphi\|_{C_{\alpha}}-N_{\alpha} D \frac{\left(\rho^{*}-\sigma\right)^{1-\alpha}}{1-\alpha}\right]^{p} } \\
& \leq \quad\left[N_{\alpha} D \frac{\left(\rho^{*}-\sigma\right)^{(1-\alpha q) / q}}{(1-\alpha q)^{1 / q}}\right]^{p} \\
& \cdot \quad \int_{\sigma}^{t} 2^{p}\left\{\left[\left\|u_{s}\right\|_{C_{\alpha}}-M\|\varphi\|_{C_{\alpha}}-N_{\alpha} D \frac{\left(\rho^{*}-\sigma\right)^{1-\alpha}}{1-\alpha}\right]^{p}\right. \\
&\left.+\quad\left[M\|\varphi\|_{C_{\alpha}}+N_{\alpha} D \frac{\left(\rho^{*}-\sigma\right)^{1-\alpha}}{1-\alpha}\right]^{p}\right\} d s \\
& \leq \quad\left[2 N_{\alpha} D \frac{\left(\rho^{*}-\sigma\right)^{(1-\alpha q) / q}}{(1-\alpha q)^{1 / q}}\right]^{p} \\
& \cdot \quad\left[M\|\varphi\|_{C_{\alpha}}+N_{\alpha} D \frac{\left(\rho^{*}-\sigma\right)^{1-\alpha}}{1-\alpha}\right]^{p}\left(\rho^{*}-\sigma\right) \\
&+\left[2 N_{\alpha} D \frac{\left(\rho^{*}-\sigma\right)^{(1-\alpha q) / q}}{(1-\alpha q)^{1 / q}}\right]^{p} \\
& . \int_{\sigma}^{t}\left[\left\|u_{s}\right\|_{C_{\alpha}}-M\|\varphi\|_{C_{\alpha}}-N_{\alpha} D \frac{\left(\rho^{*}-\sigma\right)^{1-\alpha}}{1-\alpha}\right]^{p} d s
\end{aligned}
$$

e da desigualdade de Gronwall segue que

$$
\begin{aligned}
& {\left[\left\|u_{t}\right\|_{C_{\alpha}}-M\|\varphi\|_{C_{\alpha}}-N_{\alpha} D \frac{\left(\rho^{*}-\sigma\right)^{1-\alpha}}{1-\alpha}\right]^{p}} \\
& \leq \quad\left[2 N_{\alpha} D \frac{\left(\rho^{*}-\sigma\right)^{(1-\alpha q) / q}}{(1-\alpha q)^{1 / q}}\right]^{p} \\
& \cdot \quad\left[M\|\varphi\|_{C_{\alpha}}+N_{\alpha} D \frac{\left(\rho^{*}-\sigma\right)^{1-\alpha}}{1-\alpha}\right]^{p}\left(\rho^{*}-\sigma\right) \\
& \cdot \exp \left\{\left[2 N_{\alpha} D \frac{\left(\rho^{*}-\sigma\right)^{(1-\alpha q) / q}}{(1-\alpha q)^{1 / q}}\right]^{p}\left(\rho^{*}-\sigma\right)\right\},
\end{aligned}
$$

o que implica,

$$
\left\|u_{t}\right\|_{C_{\alpha}} \leq M\|\varphi\|_{C_{\alpha}}+N_{\alpha} D \frac{\left(\rho^{*}-\sigma\right)^{1-\alpha}}{1-\alpha}
$$




$$
\begin{aligned}
+ & 2 N_{\alpha} D \frac{\left(\rho^{*}-\sigma\right)^{(1-\alpha q) / q}}{(1-\alpha q)^{1 / q}}\left(\rho^{*}-\sigma\right)^{1 / p} \\
\cdot & {\left[M\|\varphi\|_{C_{\alpha}}+N_{\alpha} D \frac{\left(\rho^{*}-\sigma\right)^{1-\alpha}}{1-\alpha}\right] } \\
\cdot & \exp \left\{2 N_{\alpha} D \frac{\left(\rho^{*}-\sigma\right)^{(1-\alpha q) / q}}{(1-\alpha q)^{1 / q}}\left(\rho^{*}-\sigma\right)^{1 / p}\right\},
\end{aligned}
$$

e assim $\left\|u_{t}\right\|_{c_{\alpha}}$ é limitada para todo $\sigma \leq t<\rho^{*}$.

Utilizando os resultados acima, ternos que existe $D_{1}>0$ tal que $\left\|F\left(t, u_{t}\right)\right\|_{X} \leq D_{1}$ para todo $\sigma \leq t<\rho^{*}$.

Devemos agora mostrar que existe $\lim _{t \rightarrow p_{-}^{*}} u(t)$ e para isto vamos usar o critério de Cauchy, ou seja, vamos mostrar que $\lim _{t, \tau \rightarrow \rho_{-}^{*}}\|u(t)-u(\tau)\|_{X^{\alpha}}=0$.

Dado $\varepsilon>0$ seja $0<\varepsilon_{1}<\rho^{*}-\sigma$ com $\varepsilon_{1}^{1-\alpha} \leq \frac{(1-\alpha) \varepsilon}{4 N_{\alpha} D_{1}}$. Além disso, sejam $t^{*}=\rho^{*}-\varepsilon_{1}$ e $0<\delta \leq \varepsilon_{1}$ tal que $\left\|\left(e^{-A\left(\tau-t^{*}\right)}-e^{-A\left(t-t^{*}\right)}\right) u\left(t^{*}\right)\right\|_{X^{\alpha}} \leq \frac{\varepsilon}{2}$ se $|t-\tau| \leq \delta$

Consideremos $u$ como solução da equação integral (1.3)

$$
u(t)=e^{-A(t-\sigma)} \varphi(0)+\int_{\sigma}^{t} e^{-A(t-s)} F\left(s, u_{s}\right) d s
$$

com condição inicial $\left(t^{*}, u_{t^{*}}\right)$, então, para $t^{*} \leq \rho^{*}-\delta \leq t, \tau<\rho^{*}$, podemos escrever

$$
u(t)=e^{-A\left(t-t^{*}\right)} u\left(t^{*}\right)+\int_{t^{*}}^{t} e^{-A(t-s)} F\left(s, u_{s}\right) d s
$$

e

$$
u(\tau)=e^{-A\left(\tau-t^{*}\right)} u\left(t^{*}\right)+\int_{t^{*}}^{\tau} e^{-A(\tau-s)} F\left(s, u_{s}\right) d s
$$

Assim,

$$
\begin{aligned}
\|u(t)-u(\tau)\|_{X^{\alpha}} & \leq \frac{\varepsilon}{2}+\frac{N_{\alpha} D_{1}}{1-\alpha}\left(t-t^{*}\right)^{1-\alpha}+\frac{N_{\alpha} D_{1}}{1-\alpha}\left(\tau-t^{*}\right)^{1-\alpha} \\
& \leq \frac{\varepsilon}{2}+\frac{2 N_{\alpha} D_{1}}{1-\alpha}\left(\rho^{*}-t^{*}\right)^{1-\alpha} \\
& =\varepsilon .
\end{aligned}
$$


Como $\varepsilon$ é arbitrário segue, usando o critério de Cauchy, que existe $\lim _{t \rightarrow \rho_{-}^{*}} u(t)=u_{1} \in X^{\alpha}$.

Para concluir, consideremos a função $\tilde{u}:\left[\rho^{*}-r, \rho^{*}\right] \rightarrow X^{\alpha}$ definida por

$$
\tilde{u}(t)= \begin{cases}u(t), & \text { se } \rho^{*}-r \leq t<\rho^{*} \\ u_{1}, & \text { se } t=\rho^{*}\end{cases}
$$

Temos assim que $\tilde{u}$ é contínua e considerando $\left(\rho^{*}, \tilde{u}_{\rho^{*}}\right)$ como condição inicial para solução da equação integral (1.3) obtemos uma solução contínua $v:\left[\rho^{*}-r, \rho^{*}+\tau\right] \rightarrow X^{\alpha}$, para algum $\tau>0$, que satisfaz $v_{\rho^{*}}=\tilde{u}_{\rho^{*}}$.

Assim, a função $u^{*}:\left[\sigma-r, \rho^{*}+\tau\right] \rightarrow X^{\alpha}$ definida por

$$
u^{*}(t)=\left\{\begin{array}{lll}
u(t), & \text { se } & \sigma-r \leq t<\rho^{*} \\
v(t), & \text { se } & \rho^{*} \leq t \leq \rho^{*}+\tau
\end{array}\right.
$$

é solução da equação integral (1.3) com $u_{\sigma}^{*}=\varphi$, contrariando o fato de $u(\sigma, \varphi)$ ser solução maximal.

Corolário 1.10 Sejam $F: \mathbb{R} \times C\left([-r, 0], X^{\alpha}\right) \rightarrow X$ contínua e localmente Lipschitz contínua em seu segundo argumento, $F(B)$ um conjunto limitado de $X$ para qualquer conjunto limitado $B$ contido em $\mathbb{R} \times C\left([-r, 0], X^{\alpha}\right)$ e $u:\left[\sigma-r, \rho^{*}\right) \rightarrow X^{\alpha}$ uma solução maximal da equação integral (1.3)

$$
u(t)=e^{-A(t-\sigma)} \varphi(0)+\int_{\sigma}^{t} e^{-A(t-s)} F\left(s, u_{s}\right) d s
$$

satisfazendo $u_{\sigma}=\varphi$. Se $\rho^{*}<\infty$ então $\lim _{t \rightarrow \rho_{-}^{*}} \sup \left\|u_{t}(\sigma, \varphi)\right\|_{C_{\alpha}}=\infty$

\section{Demonstração}

Suponhamos, por absurdo, que $\left\|u_{t}\right\|_{C_{\alpha}} \leq K$ para todo $\sigma \leq t<\rho^{*}$ e alguma constante $K$ positiva. Então $\left\{\left(t, u_{t}\right) ; \sigma \leq t<\rho^{*}\right\}$ é um conjunto limitado de $\mathbb{R} \times C\left([-r, 0], X^{\alpha}\right)$. 
Logo, $F\left(t, u_{t}\right)$ é um conjunto limitado em $X$ para todo $\sigma \leq t<\rho^{*}$, o que contradiz o lema anterior.

Teorema 1.11 Sejam. $F: \mathbb{R} \times C\left([-r, 0], X^{\alpha}\right) \rightarrow X$ contínua e localmente Lipschitz contínua em seu segundo argumento, $F(B)$ um conjunto limitado de $X$ para qualquer conjunto limitado $B$ contido em $\mathbb{R} \times C\left([-r, 0], X^{\alpha}\right)$ e $k_{1} e$ $k_{2}$ funções localmente integráveis tal que $\|F(t, \varphi)\|_{X} \leq k_{1}(t)\|\varphi\|_{C_{\alpha}}+k_{2}(t)$. Então a equação integral (1.2)

$$
u(t)=e^{-A t} \varphi(0)+\int_{0}^{t} e^{-A(t-s)} F\left(s, u_{s}\right) d s,
$$

para todo $t>0$ com $u(t)=\varphi(t)$ se $-r \leq t \leq 0$, possui solução global.

A demonstração deste teorema pode ser encontrada em Wu [31], e a idéia é mostrar que existe uma função contínua $h$ tal que $\left\|u_{t}(\varphi)\right\|_{C_{\alpha}} \leq h(t)$ e usar o corolário 1.10 .

Vamos agora considerar $F: \mathbb{R} \times C\left([-r, 0], X^{\alpha}\right) \rightarrow X$ localmente Hölder contínua em $t \in \mathbb{R}$ e localmente Lipschitz contínua em $\varphi \in C\left([-r, 0], X^{\alpha}\right)$, assim temos que uma solução da equação integral (1.2)

$$
u(t)=e^{-A t} \varphi(0)+\int_{0}^{t} e^{-A(t-s)} F\left(s, u_{s}\right) d s,
$$

para todo $t>0$, será uma solução da equação (1.1),

$$
\dot{u}(t)+A u(t)=F\left(t, u_{t}\right), \text { para todo } t>0,
$$

como nos afirma o teorema 1.14 .

Necessitamos do lema abaixo e para sua demonstração veja Henry [13]. 
Lema 1.12 Seja $h:(0, T) \rightarrow X$ localmente Hölder continua satisfazendo $\int_{0}^{\rho}\|h(s)\| d s<\infty$ para algum $\rho>0$ e seja $H(t)=\int_{0}^{t} e^{-A(t-s)} h(s) d s p a-$ ra $0 \leq t<T$. Então $H$ é contínua em $[0, T)$, continuamente diferenciável em $(0, T)$ com $H(t) \in D(A)$ para $0<t<T e \frac{d H(t)}{d t}+A H(t)=f(t)$ para $0<t<T$ e $H(t) \rightarrow 0$ em $X$ quando $t \rightarrow 0_{+}$.

A seguir consideramos $u:[-r, T] \rightarrow X^{\alpha}$ uma solução da equação integral (1.2) em $[0, T]$ com condição inicial $u_{0}=\varphi$; e julgamos oportuno comentar que nas demonstrações restantes desta seção usamos o teorema 1.5.

Teorema 1.13 Seja $\varphi:[-r, 0] \rightarrow X^{\alpha}$ localmente Hölder contínua em $(-r, 0]$ satisfazendo $\varphi(0) \in D\left(A^{\alpha+\beta}\right)$ para algum $0<\beta<1$. Então as funções $t \mapsto u(t):(-r, T] \rightarrow X^{\alpha}$ e $t \mapsto F\left(t, u_{t}\right):(0, T] \rightarrow X$ são localmente Hölder contínuas e portanto a função $t \mapsto u(t)$ é de classe $C^{1}$ em $0<t<T$.

\section{Demonstração}

Como $\varphi$ é localmente Hölder contínua e $F$ é localmente Hölder contínua em $t$ e localmente Lipschitz contínua em $\varphi$ existem $0<\delta \leq 1$ e $L, D$ e $D_{s}$ constantes positivas tais que

$$
\left\|\varphi\left(\theta_{1}\right)-\varphi\left(\theta_{2}\right)\right\|_{X^{\alpha}} \leq D_{s}\left|\theta_{1}-\theta_{2}\right|^{\delta}
$$

para $\theta_{1}, \theta_{2} \in[s, 0] \operatorname{com}-r<s \leq 0$,

$$
\left\|F\left(t_{1}, \varphi_{1}\right)-F\left(t_{2}, \varphi_{2}\right)\right\|_{X} \leq L\left(\left|t_{1}-t_{2}\right|^{\delta}+\left\|\varphi_{1}-\varphi_{2}\right\|_{C_{\alpha}}\right)
$$

e

$$
\left\|F\left(t, \varphi_{1}\right)\right\|_{X} \leq D
$$

para $\left(t_{1}, \varphi_{1}\right)$ e $\left(t_{2}, \varphi_{2}\right)$ em uma vizinhança da curva $\left\{\left(t, u_{t}\right) ; 0 \leq t \leq T\right\}$ 
Mostramos, a princípio, que $u$ é localmente Hölder contínua em $[s, T]$ para cada $-r<s \leq 0$. Para isto, observemos que para $0<t<t+h \leq T$

$$
\begin{aligned}
& u(t+h)-u(t) \\
& =e^{-A(t+h)} \varphi(0)+\int_{0}^{t+h} e^{-A(t+h-s)} F\left(s, u_{s}\right) d s \\
& -e^{-A t} \varphi(0)-\int_{0}^{t} e^{-A(t-s)} F\left(s, u_{s}\right) d s \\
& =\left(e^{-A h}-I\right) e^{-A t} \varphi(0) \\
& +\int_{0}^{t}\left(e^{-A h}-I\right) e^{-A(t-s)} F\left(s, u_{s}\right) d s \\
& +\int_{t}^{t+h} e^{-A(t+h-s)} F\left(s, u_{s}\right) d s .
\end{aligned}
$$

Dessa forma, para $s \leq t \leq t+h \leq T$ e $0<h \leq-s$, temos

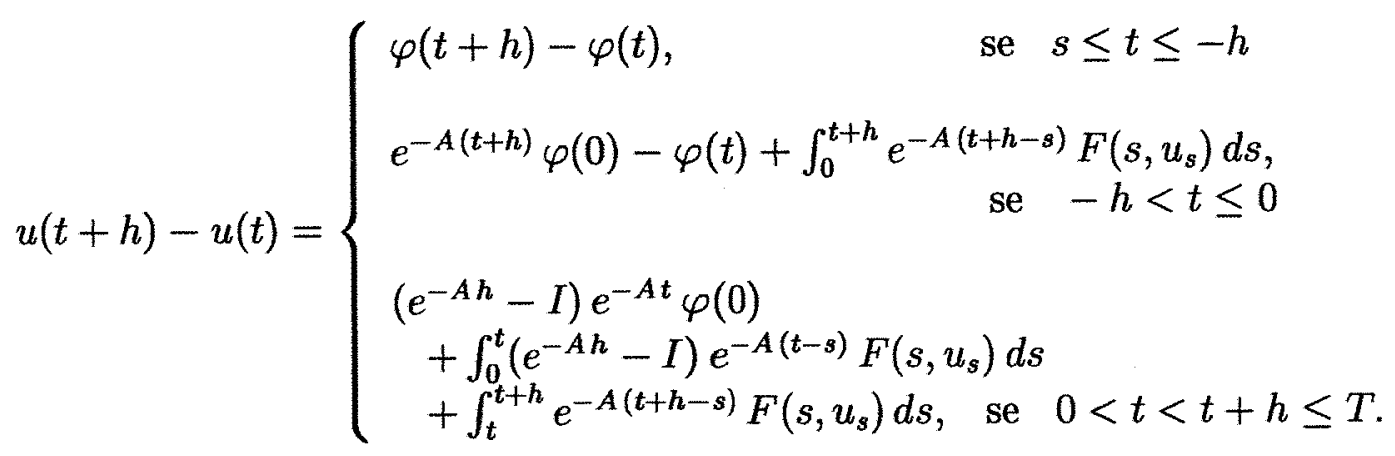

Portanto,

a) $s \leq t \leq-h$

$$
\begin{aligned}
\|u(t+h)-u(t)\|_{X^{\alpha}} & =\|\varphi(t+h)-\varphi(t)\|_{X^{\alpha}} \\
& \leq D_{s} h^{\delta}
\end{aligned}
$$

b) $-h<t \leq 0$

$$
\begin{aligned}
\|u(t+h)-u(t)\|_{X^{\alpha}} & \leq\left\|\left(e^{-A(t+h)}-I\right) \varphi(0)\right\|_{X^{\alpha}}+\|\varphi(0)-\varphi(t)\|_{X^{\alpha}} \\
& +N_{\alpha} D \frac{(t+h)^{1-\alpha}}{1-\alpha} \\
& \leq \frac{1}{\beta} N_{1-\beta}(t+h)^{\beta}\|\varphi(0)\|_{X^{\alpha+\beta}}+D_{s}|t|^{\delta}
\end{aligned}
$$




$$
\begin{aligned}
& +N_{\alpha} D \frac{(t+h)^{1-\alpha}}{1-\alpha} \\
& \leq \frac{1}{\beta} N_{1-\beta} h^{\beta}\|\varphi(0)\|_{X^{\alpha+\beta}}+D_{s} h^{\delta}+\frac{N_{\alpha} D}{1-\alpha} h^{1-\alpha} .
\end{aligned}
$$

c) $0<t \leq T$

Seja $0<\eta<1-\alpha$, então

$$
\begin{aligned}
\|u(t+h)-u(t)\|_{X^{\alpha}} & \leq\left\|\left(e^{-A h}-I\right) e^{-A t} \varphi(0)\right\|_{X^{\alpha}} \\
& +\int_{0}^{t}\left\|\left(e^{-A h}-I\right) e^{-A(t-s)} F\left(s, u_{s}\right)\right\|_{X^{\alpha}} d s \\
& +\int_{t}^{t+h}\left\|e^{-A(t+h-s)} F\left(s, u_{s}\right)\right\|_{X^{\alpha}} d s \\
& \leq \frac{1}{\beta} N_{1-\beta} M h^{\beta}\|\varphi(0)\|_{X^{\alpha+\beta}} \\
& +\int_{0}^{t} \frac{1}{\eta} N_{1-\eta} h^{\eta} N_{\alpha+\eta}(t-s)^{-(\eta+\alpha)}\left\|F\left(s, u_{s}\right)\right\|_{X} d s \\
& +N_{\alpha} D \frac{h^{1-\alpha}}{1-\alpha} \\
& \leq \frac{1}{\beta} N_{1-\beta} M h^{\beta}\|\varphi(0)\|_{X^{\alpha+\beta}} \\
& +\frac{1}{\eta} N_{1-\eta} N_{\alpha+\eta} D h^{\eta} \frac{t^{1-\eta-\alpha}}{1-\eta-\alpha} \\
& +N_{\alpha} D \frac{h^{1-\alpha}}{1-\alpha} .
\end{aligned}
$$

Assim, acabamos de verificar que

$$
\begin{aligned}
\sup _{s \leq t \leq T-h}\|u(t+h)-u(t)\|_{X^{\alpha}} \\
\leq \max \left\{D_{s} h^{\delta}, \frac{1}{\beta} N_{1-\beta}\|\varphi(0)\|_{X^{\alpha+\beta}} h^{\beta}+D_{s} h^{\delta}+\frac{N_{\alpha} D}{1-\alpha} h^{1-\alpha},\right. \\
\left.\quad \frac{1}{\beta} N_{1-\beta} M\|\varphi(0)\|_{X^{\alpha+\beta}} h^{\beta}+\frac{1}{\eta} N_{1-\eta} N_{\alpha+\eta} D \frac{T^{1-\eta-\alpha}}{1-\eta-\alpha} h^{\eta}+\frac{N_{\alpha} D}{1-\alpha} h^{1-\alpha}\right\} \\
\leq N h^{\nu},
\end{aligned}
$$

sendo $\nu=\min \{\delta, \beta, \eta\}$ e $N$ uma constante, o que nos permite concluir que $u$ é localmente Hölder contínua em $(-r, T]$.

Observemos agora que $t \mapsto u_{t}:(0, T] \rightarrow C\left([-r, 0], X^{\alpha}\right)$ é localmente 
Hölder contínua. De fato,

$$
\begin{aligned}
\left\|u_{t+h}-u_{t}\right\|_{c_{\alpha}} & =\sup _{-r \leq \theta \leq 0}\left\|u_{t+h}(\theta)-u_{t}(\theta)\right\|_{X^{\alpha}} \\
& =\sup _{t-r \leq \tau \leq t \leq T-h}\|u(\tau+h)-u(\tau)\|_{X^{\alpha}} \\
& \leq N h^{\nu}
\end{aligned}
$$

para $0<t \leq T$.

Assim, para $0<t \leq T$ temos

$$
\begin{aligned}
\left\|F\left(t+h, u_{t+h}\right)-F\left(t, u_{t}\right)\right\|_{X} & \leq L\left(h^{\delta}+\left\|u_{t+h}-u_{t}\right\|_{c_{\alpha}}\right) \\
& \leq h^{\nu}\left(L h^{\delta-\nu}+N L\right)
\end{aligned}
$$

ou seja, $t \mapsto F\left(t, u_{t}\right)$ é localmente Hölder contínua em $(0, T]$.

Pelo lema 1.12, $t \mapsto \int_{0}^{t} e^{-A(t-s)} F\left(s, u_{s}\right) d s$ é de classe $C^{1}$ em $(0, T)$ e como $t \mapsto e^{-A t} \varphi(0)$ é de classe $C^{1}$, segue que $u:(0, T) \rightarrow X^{\alpha}$ é de classe $C^{1}$.

Lembremos que $u:[-r, T] \rightarrow X^{\alpha}$ é uma solução da equação integral (1.2) em $[0, T]$ com condição inicial $u_{0}=\varphi$ e $F: \mathbb{R} \times C\left([-r, 0], X^{\alpha}\right) \rightarrow X$ é localmente Hölder contínua em $t \in \mathbb{R}$ e localmente Lipschitz contínua em $\varphi \in C\left([-r, 0], X^{\alpha}\right)$

Teorema 1.14 Sejam $\varphi:[-r, 0] \rightarrow X^{\alpha}$ contínua e $T>r$. Então u é localmente Hölder contínua em $(0, r]$ e $u(r) \in D\left(A^{\alpha+\beta}\right)$ para todo $0<\beta<1-\alpha$ e portanto a função $u$ é de classe $C^{1}$ em $r<t<T$.

\section{Demonstração}

Como observamos no teorema 1.13 acima, para $0<t<t+h \leq T$, temos

$$
u(t+h)-u(t)=\left(e^{-A h}-I\right) e^{-A t} \varphi(0)
$$




$$
\begin{aligned}
& +\int_{0}^{t}\left(e^{-A h}-I\right) e^{-A(t-s)} F\left(s, u_{s}\right) d s \\
& +\int_{t}^{t+h} e^{-A(t+h-s)} F\left(s, u_{s}\right) d s
\end{aligned}
$$

Como conseqüência, pois $0<\beta<1-\alpha$,

$$
\begin{aligned}
\|u(t+h)-u(t)\|_{X^{\alpha}} & \leq\left\|\left(e^{-A h}-I\right) e^{-A t} \varphi(0)\right\|_{X^{\alpha}} \\
& +\int_{0}^{t}\left\|\left(e^{-A h}-I\right) e^{-A(t-s)} F\left(s, u_{s}\right)\right\|_{X^{\alpha}} d s \\
& +\int_{t}^{t+h}\left\|e^{-A(t+h-s)} F\left(s, u_{s}\right)\right\|_{X^{\alpha}} d s \\
& \leq \frac{1}{\beta} N_{1-\beta} h^{\beta} N_{\beta} t^{-\beta}\|\varphi(0)\|_{X^{\alpha}} \\
& +\int_{0}^{t} \frac{1}{\beta} N_{1-\beta} h^{\beta} N_{\alpha+\beta}(t-s)^{-(\alpha+\beta)}\left\|F\left(s, u_{s}\right)\right\|_{X} d s \\
& +\int_{t}^{t+h} N_{\alpha}(t+h-s)^{-\alpha}\left\|F\left(s, u_{s}\right)\right\|_{X} d s \\
& \leq \frac{1}{\beta} N_{1-\beta} N_{\beta} h^{\beta} t^{-\beta}\|\varphi(0)\|_{X^{\alpha}} \\
& +\frac{1}{\beta} N_{1-\beta} D N_{\alpha+\beta} h^{\beta} \frac{t^{1-\alpha-\beta}}{1-\alpha-\beta}+N_{\alpha} D \frac{h^{1-\alpha}}{1-\alpha} \\
& =N h^{\beta} t^{-\beta}
\end{aligned}
$$

para alguma constante $N$, o que implica que $u$ é localmente Hölder contínua em $(0, T]$.

Notemos também que $u(r) \in D\left(A^{\alpha+\beta}\right)$, pois

$$
u(r)=e^{-A r} \varphi(0)+\int_{0}^{r} e^{-A(r-s)} F\left(s, u_{s}\right) d s
$$

e portanto,

$$
\begin{aligned}
\|u(r)\|_{X^{\alpha+\beta}} & \leq N_{\beta} r^{-\beta}\|\varphi(0)\|_{X^{\alpha}}+\frac{N_{\alpha+\beta} D}{1-\alpha-\beta} r^{1-\alpha-\beta} \\
& <\infty
\end{aligned}
$$

para todo $0<\beta<1-\alpha$. 
A seguir, mostramos que $t \mapsto F\left(t, u_{t}\right)$ é localmente Hölder contínua em $(r, T]$. De fato,

$$
\begin{aligned}
\left\|u_{t+h}-u_{t}\right\|_{C^{\alpha}} & =\sup _{-r \leq \theta \leq 0}\left\|u_{t+h}(\theta)-u_{t}(\theta)\right\|_{X^{\alpha}} \\
& =\sup _{t-r \leq \tau \leq t}\|u(\tau+h)-u(\tau)\|_{X^{\alpha}} \\
& \leq \sup _{t-r \leq r \leq t} N h^{\beta} \tau^{-\beta} \\
& \leq N h^{\beta}(s-r)^{-\beta}
\end{aligned}
$$

para $r<s \leq t \leq T$.

Assim, para $r<s \leq t<t+h \leq T$,

$$
\begin{aligned}
\left\|F\left(t+h, u_{t+h}\right)-F\left(t, u_{t}\right)\right\|_{X} & \leq L\left(h^{\delta}+\left\|u_{t+h}-u_{t}\right\|_{C^{\alpha}}\right) \\
& \leq C_{s} h^{\nu}
\end{aligned}
$$

sendo $\nu=\min \{\delta, \beta\}$.

Pelo lema 1.12, $t \mapsto \int_{r}^{t} e^{-A(t-s)} F\left(s, u_{s}\right) d s$ é de classe $C^{1}$ em $(r, T) \mathrm{e}$ como $t \mapsto e^{-A t} \varphi(0)$ é de classe $C^{1}$, segue que $u:(r, T) \rightarrow X^{\alpha}$ é de classe $C^{1}$.

Vamos agora obter informações adicionais sobre a regularidade das soluções da equação (1.1)

$$
\dot{u}(t)+A u(t)=F\left(t, u_{t}\right), \text { para todo } t \geq 0
$$

Teorema 1.15 Sejam $F: \mathbb{R} \times C\left([-r, 0], X^{\alpha}\right) \rightarrow X$ localmente Hölder contínua em $t \in \mathbb{R}$, com expoente $0<\delta \leq 1$, e localmente Lipschitz contínua em seu segundo argumento e $\varphi:[-r, 0] \rightarrow X^{\alpha}$ contínua. Então qualquer solução $u:(-r, T] \rightarrow X^{\alpha}$ de $\dot{u}(t)+A u(t)=F\left(t, u_{t}\right)$, com condição inicial 
$u_{0}=\varphi$, é tal que $\frac{d u(t)}{d t} \in X^{\gamma}$ para todo $0 \leq \gamma<\nu, \operatorname{com} \nu=\min \{\delta, 1-\alpha\} e$ $\left\|\frac{d u(t)}{d t}\right\|_{X^{\gamma}}=O\left((t-r)^{\alpha-\gamma-1}\right)$ quando $t \rightarrow r$.

\section{Demonstração}

Como $F$ é localmente Hölder contínua em $t$, com expoente $0<\delta \leq 1$, e localmente Lipschitz contínua em seu segundo argumento, existem $D$ e $L$ constantes positivas tais que

$$
\left\|F\left(t_{1}, \varphi_{1}\right)-F\left(t_{2}, \varphi_{2}\right)\right\|_{X} \leq L\left(\left|t_{1}-t_{2}\right|^{\delta}+\left\|\varphi_{1}-\varphi_{2}\right\|_{c_{\alpha}}\right)
$$

$\mathrm{e}$

$$
\left\|F\left(t, \varphi_{1}\right)\right\|_{X} \leq D
$$

para $\left(t_{1}, \varphi_{1}\right)$ e $\left(t_{2}, \varphi_{2}\right)$ em uma vizinhança da curva $\left\{\left(t, u_{t}\right) ; 0 \leq t \leq T\right\}$.

Definindo $g(t)=F\left(t, u_{t}\right)$ segue, da demonstração do teorema 1.14, que

$$
\|g(t+h)-g(t)\|_{x} \leq C_{s} h^{\nu}
$$

para $r<s \leq t<t+h \leq T$ e $\nu=\min \{\delta, 1-\alpha\}$ e disto resulta que

$$
\begin{aligned}
-A \int_{r}^{t} e^{-A(t-s)} g(s) d s & =-\int_{r}^{t} A e^{-A(t-s)}(g(s)-g(t)) d s \\
& +e^{-A(t-r)} g(t)-g(t) .
\end{aligned}
$$

De fato,

$$
\begin{aligned}
& \left(\frac{e^{-A h}-I}{h}\right)\left(\int_{r}^{t} e^{-A(t-s)} g(s) d s\right) \\
& =h^{-1} \int_{r}^{t}\left(e^{-A h}-I\right) e^{-A(t-s)}(g(s)-g(t)) d s \\
& +h^{-1} \int_{r}^{r+h} e^{-A(t+h-s)} g(t) d s \\
& -h^{-1} \int_{t-h}^{t} e^{-A(t-s)} g(t) d s
\end{aligned}
$$


sendo os dois últimos termos uniformemente convergentes em $t$ e quanto ao primeiro termo, para $0<\varepsilon<\nu$, temos

$$
\begin{aligned}
& \left\|h^{-1} \int_{r}^{t}\left(e^{-A h}-I+h A\right) e^{-A(t-s)}(g(s)-g(t)) d s\right\|_{X} \\
& \quad=\left\|\int_{r}^{t} h^{-1} \int_{0}^{h} A\left(I-e^{-A \sigma}\right) e^{-A(t-s)}(g(s)-g(t)) d \sigma d s\right\|_{X} \\
& \quad \leq \int_{r}^{t} h^{-1} \int_{0}^{h} \frac{1}{\varepsilon} N_{1-\varepsilon} \sigma^{\varepsilon}\left\|A^{1+\varepsilon} e^{-A(t-s)}(g(s)-g(t))\right\|_{X} d \sigma d s \\
& \quad \leq \frac{1}{\varepsilon} N_{1-\varepsilon} N_{1+\varepsilon} C_{s} \frac{h^{\varepsilon}}{1+\varepsilon} \frac{(t-r)^{-\varepsilon+\nu}}{-\varepsilon+\nu},
\end{aligned}
$$

que converge a zero uniformemente para $r \leq t \leq T$ quando $h \rightarrow 0_{+}$.

Assim, acabamos de verificar que

$$
\begin{aligned}
-A \int_{r}^{t} e^{-A(t-s)} g(s) d s & =-\int_{r}^{t} A e^{-A(t-s)}(g(s)-g(t)) d s \\
& +e^{-A(t-r)} g(t)-g(t)
\end{aligned}
$$

Como conseqüência temos que

$$
\begin{aligned}
\frac{d u(t)}{d t} & =-A u(t)+g(t) \\
& =-A e^{-A(t-r)} \varphi(0)+e^{-A(t-r)} g(t) \\
& +\int_{r}^{t} A e^{-A(t-s)}(g(t)-g(s)) d s
\end{aligned}
$$

e portanto,

$$
\begin{aligned}
\left\|\frac{d u(t)}{d t}\right\|_{X^{\gamma}} & \leq\left\|-A e^{-A(t-r)} \varphi(0)\right\|_{X^{\gamma}}+\left\|e^{-A(t-r)} g(t)\right\|_{X^{\gamma}} \\
& +\int_{\tau}^{t}\left\|A e^{-A(t-s)}(g(t)-g(s))\right\|_{X^{\gamma}} d s \\
& \leq N_{\gamma+1-\alpha}(t-r)^{-(\gamma+1-\alpha)}\|\varphi(0)\|_{X^{\alpha}} \\
& +N_{\gamma}(t-r)^{-\gamma} D \\
& +\int_{r}^{t} N_{\gamma+1}(t-s)^{-(\gamma+1)} C_{s}(t-s)^{\nu} d s \\
& \leq N_{\gamma+1-\alpha}(t-r)^{-(\gamma+1-\alpha)}\|\varphi(0)\|_{X^{\alpha}}+N_{\gamma} D(t-r)^{-\gamma}
\end{aligned}
$$




$$
\begin{aligned}
& +\frac{1}{-\gamma+\nu} N_{\gamma+1} C_{s}(t-r)^{-\gamma+\nu} \\
& =O\left((t-r)^{\alpha-\gamma-1}\right)
\end{aligned}
$$

quando $t \rightarrow r$.

Os resultados a seguir nos mostram que, para $F$ autônoma e localmente Lipschitz contínua, as soluções da equação integral (1.2)

$$
u(t)=e^{-A t} \varphi(0)+\int_{0}^{t} e^{-A(t-s)} F\left(u_{s}\right) d s
$$

para todo $t>0 \mathrm{com} u(t)=\varphi(t)$ se $-r \leq t \leq 0$, definem um semigrupo fortemente contínuo.

Neste caso, se $u:\left[-r, \rho^{*}(\varphi)\right) \rightarrow X^{\alpha}$ é uma solução maximal da equação integral (1.2) e $s \in\left[0, \rho^{*}(\varphi)\right)$ então a função $v:\left[-s-r, \rho^{*}(\varphi)-s\right) \rightarrow X^{\alpha}$ definida por $v(t)=u(\varphi)(t+s)$ é uma solução da equação integral (1.2) satisfazendo $v_{0}=u_{s}(\varphi)$.

De fato,

$$
\begin{aligned}
v(t) & =u(\varphi)(t+s) \\
& =e^{-A(t+s)} \varphi(0)+\int_{0}^{t+s} e^{-A(t+s-\sigma)} F\left(u_{\sigma}\right) d \sigma \\
& =e^{-A t}\left[e^{-A s} \varphi(0)+\int_{0}^{s} e^{-A(s-\sigma)} F\left(u_{\sigma}\right) d \sigma\right] \\
& +\int_{s}^{t+s} e^{-A(t+s-\sigma)} F\left(u_{\sigma}\right) d \sigma \\
& =e^{-A t} u(\varphi)(s)+\int_{0}^{t} e^{-A(t-\sigma)} F\left(u_{\sigma+s}\right) d \sigma \\
& =e^{-A t} u_{s}(\varphi)(0)+\int_{0}^{t} e^{-A(t-\sigma)} F\left(v_{\sigma}\right) d \sigma .
\end{aligned}
$$

Logo, pela unicidade de soluções da equação integral (1.2), temos que $v(t)=u\left(u_{s}(\varphi)\right)(t)$ para todo $t \in\left[-r, \rho^{*}\left(u_{s}(\varphi)\right)\right)$. E usando o fato de $u$ ser 
solução maximal segue que $\rho^{*}(\varphi)-s \leq \rho^{*}\left(u_{s}(\varphi)\right)$ e $u(\varphi)(t+s)=u\left(u_{s}(\varphi)\right)(t)$ para todo $t, s \geq 0$ com $s-r \leq t+s<\rho^{*}(\varphi)$.

Assim, para $-r \leq \theta \leq 0$ temos

$$
\begin{aligned}
u_{t+s}(\varphi)(\theta) & =u(\varphi)(t+s+\theta)=u\left(u_{s}(\varphi)\right)(t+\theta) \\
& =u_{t}\left(u_{s}(\varphi)\right)(\theta)
\end{aligned}
$$

para todo $t, s \geq 0$ com $t+s<\rho^{*}(\varphi)$.

Observemos agora que, se as soluções $u(\varphi)$ da equação integral (1.2) estão definidas em $[-r, \infty)$, a aplicação $U(t): C\left([-r, 0], X^{\alpha}\right) \rightarrow C\left([-r, 0], X^{\alpha}\right)$ definida por $U(t) \varphi=u_{t}(\varphi)$, para todo $t \geq 0$, define um semigrupo fortemente contínuo $\{U(t) ; t \geq 0\}$ em $C\left([-r, 0], X^{\alpha}\right)$, pois

$$
U(t+s) \varphi=u_{t+s}(\varphi)=u_{t}\left(u_{s}(\varphi)\right)=U(t) u_{s}(\varphi)=U(t) U(s) \varphi
$$

e $U(0) \varphi=u_{0}(\varphi)=\varphi$, e da continuidade das soluções da equação integral (1.2) segue que o semigrupo $\{U(t) ; t \geq 0\}$ é fortemente contínuo.

Dado $t>r$, a proposição a seguir nos garante que o semigrupo $\{U(t) ; t \geq 0\}$ é condicionalmente completamente contínuo, e por conveniência enunciamos esta definição [8].

Definição 1.16 Um semigrupo $T(t): X \rightarrow X, t \geq 0, X$ espaço de Banach, é dito ser condicionalmente completamente contínuo para $t \geq t_{1}$ se para cada $t \geq t_{1}$ e cada conjunto $B \subset X$ limitado para o qual $\{T(s) B ; 0 \leq s \leq t\}$ é limitado temos $T(t) B$ pré-compacto.

Proposição $1.17 O$ semigrupo $\{U(t) ; t \geq 0\}$ é condicionalmente completamente contínuo para cada $t>r$ fixado. 


\section{Demonstração}

Seja $B \subset C\left([-r, 0], X^{\alpha}\right)$ limitado com $\{U(s) B ; 0 \leq s \leq r\}$ limitado. Queremos mostrar que $\overline{U(t) B}$ é compacto.

Temos que

$$
U(t) B=\left\{U(t) \varphi:[-r, 0] \rightarrow X^{\alpha} \text { contínua; } \varphi \in B\right\}
$$

e, pelo teorema de Arzelá-Ascoli, devemos mostrar que $U(t) B$ é eqüicontínuo e para todo $\theta \in[-r, 0],\{U(t) \varphi(\theta) ; \varphi \in B\}$ é relativamente compacto em $X^{\alpha}$, ou seja, $\{U(t) \varphi(\theta) ; \varphi \in B\}$ é limitado em $X^{\beta}$ para $0<\alpha<\beta<1$.

Para isto, seja $t>r$ fixado, mas arbitrário. Como $\frac{d u(t)}{d t}$ é limitado para $t>r$ pelo teorema 1.15 , segue que $U(t) B$ é eqüicontínuo.

E fixado $\theta \in[-r, 0]$ temos que

$$
\begin{aligned}
\|U(t) \varphi(\theta)\|_{X^{\beta}} & =\left\|u_{t}(\varphi)(\theta)\right\|_{X^{\beta}} \\
& \leq\left\|e^{-A(t+\theta)} \varphi(0)\right\|_{X^{\beta}} \\
& +\int_{0}^{t+\theta}\left\|e^{-A(t+\theta-\sigma)} F\left(u_{\sigma}\right)\right\|_{X^{\beta}} d \sigma \\
& \leq N_{\beta-\alpha}(t+\theta)^{\alpha-\beta}\|\varphi(0)\|_{X^{\alpha}} \\
& +\frac{N_{\beta} D}{1-\beta}(t+\theta)^{1-\beta} .
\end{aligned}
$$

Assim, $\{U(t) \varphi(\theta) ; \varphi \in B\}$ é um subconjunto limitado de $X^{\beta}$ e portanto, relativamente compacto em $X^{\alpha}$. 


\subsection{Existência de soluções para problemas com retardo infinito}

Vamos agora discutir alguns resultados de existência de soluçôes para problemas com retardo infinito. As principais referências que usamos foram Hale e Kato [9], Henríquez [10, 11, 12], Hino, Murakami e Naito [17] e Ruan e Wu [25]. As demonstrações são muito semelhantes às apresentadas na seção 1.1 .

Nos problemas com retardo infinito temos várias possibilidades para a escolha do espaço de fase [17]. Neste trabalho iremos usar a definição de espaço de fase introduzida por Hale e Kato [9] e seguiremos a notação usada no livro de Hino, Murakami e Naito [17] para estabelecer os axiomas do espaço de fase.

Assim, o espaço de fase $\mathcal{B}$, para equações com retardo infinito, é um espaço linear de funções $\varphi:(-\infty, 0] \rightarrow X^{\alpha}$ com uma seminorma $\|\cdot\|_{B}$ satisfazendo os seguintes axiomas:

(A1) Se $x:(-\infty, \sigma+a) \rightarrow X^{\alpha}, a>0$, é contínua em $[\sigma, \sigma+a)$ e $x_{\sigma} \in \mathcal{B}$ então para cada $t \in[\sigma, \sigma+a)$ as seguintes condições valem

(i) $x_{t} \in \mathcal{B}$;

(ii) $\|x(t)\|_{X^{\alpha}} \leq H\left\|x_{t}\right\|_{\mathcal{B}}$, sendo $H \geq 0$ constante;

(iii) $\left\|x_{t}\right\|_{\mathcal{B}} \leq K(t-\sigma) \sup _{\sigma \leq s \leq t}\|x(s)\|_{X^{\alpha}}+M(t-\sigma)\left\|x_{\sigma}\right\|_{\mathcal{B}}$, sendo $K, M:[0, \infty) \rightarrow[0, \infty), K$ contínua e $M$ localmente limitada e $K$ e $M$ independentes de $x(\cdot)$

(A2) Para a função $x(\cdot)$ em $(A 1), x_{t}$ é uma função em $\mathcal{B}$ contínua em $[\sigma, \sigma+a)$.

(A3) O espaço $\mathcal{B}$ é completo. 
Exemplos de espaços de fase para equações com retardo infinito podem ser encontrados em Hino, Murakami e Naito [17] e Henríquez [12].

Observemos que se $\varphi:(-\infty, 0] \rightarrow X^{\alpha}$ é uma função contínua com suporte compacto então $\varphi \in \mathcal{B}$. Além disso, se $\operatorname{supp} \varphi \subset[-a, 0]$, para $a>0$, segue que

$$
\|\varphi\|_{\mathcal{B}} \leq K(a) \max _{-a \leq \theta \leq 0}\|\varphi(\theta)\|_{X^{\alpha}}
$$

A demonstração deste resultado segue do axioma $(A 1)$ e pode ser encontrada em Hino, Murakami e Naito [17].

Observemos também que $\|\varphi-\psi\|_{\mathcal{B}}=0$ não significa necessariamente que $\varphi(\theta)=\psi(\theta)$ para todo $\theta \leq 0$, pois $\|\cdot\|_{\mathcal{B}}$ é uma seminorma. Mas de $(A 1 i i)$ temos que $\varphi(0)=\psi(0)$ se $\|\varphi-\psi\|_{\mathcal{B}}=0$.

Apresentamos, a seguir, detalhes quanto à existência, unicidade e dependência contínua de soluções da equação

$$
\dot{u}(t)+A u(t)=F\left(t, u_{t}\right), \text { para todo } t \geq 0,
$$

com $F: \mathbb{R} \times \mathcal{B} \rightarrow X$ contínua e localmente Lipschitz contínua em seu segundo argumento e $u_{t}:(-\infty, 0] \rightarrow X^{\alpha}$ a função definida por $u_{t}(\theta)=u(t+\theta)$.

Definição 1.18 Uma função $u:(-\infty, \sigma+a] \rightarrow X^{\alpha}$ é solução de (1.7) se $u_{\sigma}=\varphi$, a restrição $u:[\sigma, \sigma+a] \rightarrow X^{\alpha}$ é contínua e continuamente diferenciável em $(\sigma, \sigma+a), u(t) \in D(A)$ e $\dot{u}(t)+A u(t)=F\left(t, u_{t}\right)$ para todo $\sigma<t<\sigma+a$

Teorema 1.19 Seja $F: \mathbb{R} \times \mathcal{B} \rightarrow X$ contínua e localmente Lipschitz contínua em seu segundo argumento. Dado $(\sigma, \varphi) \in \mathbb{R} \times \mathcal{B}$ existem um número real 
$b=b(\sigma, \varphi)>0$ e uma única função $u:(-\infty, \sigma+b] \rightarrow X^{\alpha}$, tal que $u_{\sigma}=\varphi e$

$$
u(t)=e^{-A(t-\sigma)} \varphi(0)+\int_{\sigma}^{t} e^{-A(t-s)} F\left(s, u_{s}\right) d s
$$

para todo $\sigma \leq t \leq \sigma+b$.

\section{Demonstração}

Como $F$ é localmente Lipschitz contínua em seu segundo argumento existem $b_{1}>0, r_{1}>0$ e constantes positivas $L$ e $D$ tais que se $|t-\sigma| \leq b_{1}$, $\left\|\varphi_{1}-\varphi\right\|_{\mathcal{B}} \leq r_{1}$ e $\left\|\varphi_{2}-\varphi\right\|_{\mathcal{B}} \leq r_{1}$ então

$$
\left\|F\left(t, \varphi_{1}\right)-F\left(t, \varphi_{2}\right)\right\|_{X} \leq L\left\|\varphi_{1}-\varphi_{2}\right\|_{\mathcal{B}}
$$

e

$$
\left\|F\left(t, \varphi_{1}\right)\right\|_{X} \leq D
$$

Consideremos a função $v:\left(-\infty, \sigma+b_{1}\right] \rightarrow X^{\alpha}$ definida por

$$
v(t)= \begin{cases}e^{-A(t-\sigma)} \varphi(0), & \text { se } \sigma<t \leq \sigma+b_{1} \\ \varphi(t-\sigma), & \text { se } t \leq \sigma\end{cases}
$$

e, do axioma $(A 1 i)$, segue que $v_{t} \in \mathcal{B}$ para $\sigma<t \leq \sigma+b_{1}$, e para cada $0<r_{2}<r_{1}$ existe $0<b_{2} \leq b_{1}$ tal que $\left\|v_{t}-\varphi\right\|_{\mathcal{B}} \leq r_{2}$ para todo $\sigma<t<\sigma+b_{2}$.

Sejam $K_{a}=\max \{K(t) ; 0 \leq t \leq a\}$ e $b<b_{2} \operatorname{com} \frac{N_{\alpha} D b^{1-\alpha}}{1-\alpha} \leq \frac{r_{1}-r_{2}}{K_{b}}$ e $\frac{N_{\alpha} L K_{b} b^{1-\alpha}}{1-\alpha}<\frac{1}{2}$ e $N_{\alpha}$ dado pelo teorema 1.5 .

Consideremos agora o conjunto $S_{b}$ das funções $u:(-\infty, \sigma+b] \rightarrow X^{\alpha}$ $\operatorname{com} u_{\sigma} \in \mathcal{B}$, a restrição de $u$ ao intervalo $[\sigma, \sigma+b]$ contínua e com a seminorma

$$
\|u\|_{S_{b}}=\left\|u_{\sigma}\right\|_{\mathcal{B}}+\sup _{\sigma \leq s \leq \sigma+b}\|u(s)\|_{X^{\alpha}}
$$

Dizemos que $u \in S_{b}(\varphi)$ se $u \in S_{b},\left\|u_{\sigma}-\varphi\right\|_{\mathcal{B}}=0$ e $\left\|u_{t}-\varphi\right\|_{\mathcal{B}} \leq r_{1}$ para todo $\sigma \leq t \leq \sigma+b$. 
E definimos $G: S_{b}(\varphi) \rightarrow C\left((-\infty, \sigma+b], X^{\alpha}\right)$ por

$(G u)(t)= \begin{cases}e^{-A(t-\sigma)} \varphi(0)+\int_{\sigma}^{t} e^{-A(t-s)} F\left(s, u_{s}\right) d s, & \text { se } \sigma<t \leq \sigma+b \\ \varphi(t-\sigma), & \text { se } t \leq \sigma\end{cases}$

Devemos mostrar que $G$ tem um único ponto fixo em $S_{b}(\varphi)$ e para isto usamos o teorema do ponto fixo de Banach.

Seja $\left(u_{n}\right)$ uma seqüência em $S_{b}(\varphi)$ que converge para $u \in S_{b}$. Então, $\left\|u_{\sigma}-\varphi\right\|_{\mathcal{B}}=0$, pois $\left\|\left(u_{n}\right)_{\sigma}-\varphi\right\|_{\mathcal{B}}=0 . \mathrm{E}$, do axioma $(A 1 i i i)$, segue que

$$
\begin{aligned}
& \left\|\left(u_{n}\right)_{t}-u_{t}\right\|_{\mathcal{B}} \\
& \quad \leq K(t-\sigma) \sup _{\sigma \leq s \leq t}\left\|u_{n}(s)-u(s)\right\|_{X^{\alpha}}+M(t-\sigma)\left\|\left(u_{n}\right)_{\sigma}-u_{\sigma}\right\|_{\mathcal{B}} \\
& \leq K\left\|u_{n}-u\right\|_{S_{b}},
\end{aligned}
$$

para alguma constante $K$.

Assim, $\left(u_{n}\right)_{t} \rightarrow u_{t}$ quando $n \rightarrow \infty$ para $\sigma \leq t \leq \sigma+b$, o que implica $\left\|u_{t}-\varphi\right\|_{\mathcal{B}} \leq r_{1}$ para $\sigma \leq t \leq \sigma+b$.

Assim, temos que $S_{b}(\varphi)$ é fechado.

A fim de provarmos que $G\left(S_{b}(\varphi)\right) \subset S_{b}(\varphi)$ consideremos $u \in S_{b}(\varphi)$. Como $G u$ é contínua em $[\sigma, \sigma+b]$ e $(G u)_{\sigma}=\varphi \in \mathcal{B}$ temos que $G u \in S_{b}$.

Seja $w=G u-v$. Então, para $\sigma \leq t \leq \sigma+b$, temos

$$
\begin{aligned}
\left\|(G u)_{t}-\varphi\right\|_{\mathcal{B}} & \leq\left\|v_{t}-\varphi\right\|_{\mathcal{B}}+\left\|w_{t}\right\|_{\mathcal{B}} \\
& \leq r_{2}+\left\|w_{t}\right\|_{\mathcal{B}}
\end{aligned}
$$

$\mathrm{E}$, do axioma (A1iii), segue que

$$
\left\|w_{t}\right\|_{\mathcal{B}} \leq K(t-\sigma) \sup _{\sigma \leq s \leq t}\|w(s)\| X^{\alpha}
$$

Mas,

$$
\|w(s)\|_{X^{\alpha}} \leq \int_{\sigma}^{s}\left\|e^{-A(s-\tau)} F\left(\tau, u_{\tau}\right)\right\|_{X^{\alpha}} d \tau
$$




$$
\begin{aligned}
& =\frac{N_{\alpha} D}{1-\alpha}(s-\sigma)^{1-\alpha} \\
& \leq \frac{r_{1}-r_{2}}{K_{b}}
\end{aligned}
$$

e portanto,

$$
\begin{aligned}
\left\|(G u)_{t}-\varphi\right\|_{\mathcal{B}} & \leq r_{2}+K_{b} \frac{r_{1}-r_{2}}{K_{b}} \\
& =r_{1}
\end{aligned}
$$

para $\sigma \leq t \leq \sigma+b$, o que mostra que $G u \in S_{b}(\varphi)$.

Para concluir, mostramos que $G$ é uma contração. Com esse propósito sejam $u, v \in S_{b}(\varphi)$ e $\sigma \leq t \leq \sigma+b$. Então,

$$
\begin{aligned}
& \|(G u)(t)-(G v)(t)\|_{X^{\alpha}} \\
& \quad \leq \int_{\sigma}^{t} N_{\alpha}(t-s)^{-\alpha} L\left\|u_{s}-v_{s}\right\|_{\mathcal{B}} d s \\
& \leq N_{\alpha} L \sup _{\sigma \leq s \leq \sigma+b}\left\|u_{s}-v_{s}\right\|_{\mathcal{B}} \frac{(t-\sigma)^{1-\alpha}}{1-\alpha} \\
& \quad \leq \frac{N_{\alpha} L b^{1-\alpha}}{1-\alpha} K_{b}\|u-v\|_{S_{b}},
\end{aligned}
$$

pois,

$$
\begin{aligned}
& \left\|u_{s}-v_{s}\right\|_{\mathcal{B}} \\
& \quad \leq K(s-\sigma) \sup _{\sigma \leq \tau \leq s \leq \sigma+b}\|u(\tau)-v(\tau)\|_{X^{\alpha}}+M(s-\sigma)\left\|u_{\sigma}-v_{\sigma}\right\|_{\mathcal{B}} \\
& \quad \leq K_{b} \sup _{\sigma \leq \tau \leq \sigma+b}\|u(\tau)-v(\tau)\|_{X^{\alpha}} \\
& \leq K_{b}\|u-v\|_{S_{b}} .
\end{aligned}
$$

Portanto,

$$
\begin{aligned}
\|G u-G v\|_{S_{b}} \\
\quad=\left\|(G u)_{\sigma}-(G v)_{\sigma}\right\|_{\mathcal{B}}+\sup _{\sigma \leq t \leq \sigma+b}\|(G u)(t)-(G v)(t)\|_{X^{\alpha}} \\
\quad \leq \frac{N_{\alpha} L K_{b} b^{1-\alpha}}{1-\alpha}\|u-v\|_{S_{b}} \\
\quad \leq \frac{1}{2}\|u-v\|_{S_{b}} .
\end{aligned}
$$


Como conseqüência do teorema do ponto fixo de Banach temos que $G$ tem um único ponto fixo em $S_{b}(\varphi)$ e assim segue o resultado desejado.

Lema 1.20 Sejam $u, v:(-\infty, \sigma+a] \rightarrow X^{\alpha}$, com $a>0$, soluções contínuas da equação integral (1.8)

$$
u(t)=e^{-A(t-\sigma)} \varphi(0)+\int_{\sigma}^{t} e^{-A(t-s)} F\left(s, u_{s}\right) d s,
$$

para todo $\sigma<t \leq \sigma+a \operatorname{com} u_{\sigma}=v_{\sigma}=\varphi$. Então $u=v$ em $(-\infty, \sigma+a]$.

Como a demonstração deste lema é muito semelhante à demonstração do lema 1.7, será omitida neste caso.

Em analogia com o problema de retardo finito, o lema acima nos garante a existência de uma solução maximal para a equação (1.8).

Proposição 1.21 Seja $u=u(\sigma, \varphi)$ a solução da equação integral (1.8)

$$
u(t)=e^{-A(t-\sigma)} \varphi(0)+\int_{\sigma}^{t} e^{-A(t-s)} F\left(s, u_{s}\right) d s
$$

definida em $[\sigma, \rho)$ para algum $\rho>\sigma$ e com $u_{\sigma}=\varphi$ e seja $T$ um número real tal que $\sigma<T<\rho$. Então existe um número $\delta>0$ tal que qualquer solução $v=v(\sigma, \psi)$ da equação integral (1.8), com $v_{\sigma}=\psi e\|\varphi-\psi\|_{C_{\alpha}}<\delta$, está definida pelo menos em $[\sigma, T]$. Além disso, para $t$ fixo, $\sigma \leq t \leq T, a$ aplicação $\varphi \mapsto u_{t}(\sigma, \varphi)$ é contínua.

A demonstração desta proposição é muito semelhante à demonstração da proposição 1.8 e será omitida neste caso. 
Teorema 1.22 Sejam $F: \mathbb{R} \times \mathcal{B} \rightarrow X$ contínua e localmente Lipschitz contínua em seu segundo argumento, $F(B)$ um conjunto limitado de $X$ para qualquer conjunto limitado $B$ contido em $\mathbb{R} \times \mathcal{B}$ e $u:(-\infty, b) \rightarrow X^{\alpha}$ uma solução maximal da equação integral (1.8)

$$
u(t)=e^{-A(t-\sigma)} \varphi(0)+\int_{\sigma}^{t} e^{-A(t-s)} F\left(s, u_{s}\right) d s
$$

satisfazendo $u_{\sigma}=\varphi$. Então existe $t_{B}$ tal que $\left(t, u_{t}\right) \notin B$ para $t_{B} \leq t<b$.

A demonstração deste teorema é muito semelhante à apresentada para o lema 1.9. Convém observar que neste caso supomos, por absurdo, que $\left(t, u_{t}\right) \in B$ para todo $0<t_{0} \leq t<b$ e portanto existe uma constante positiva $D_{1}$ tal que $\left\|F\left(t, u_{t}\right)\right\|_{x} \leq D_{1}$ para todo $0 \leq t<b$.

Corolário 1.23 Sejam $F: \mathbb{R} \times \mathcal{B} \rightarrow X$ contínua e localmente Lipschitz contínua em seu segundo argumento, $F(B)$ um conjunto limitado de $X$ para qualquer conjunto limitado $B$ contido em $\mathbb{R} \times \mathcal{B}$ e $u:(-\infty, b) \rightarrow X^{\alpha}$ uma solução maximal da equação integral (1.8)

$$
u(t)=e^{-A(t-\sigma)} \varphi(0)+\int_{\sigma}^{t} e^{-A(t-s)} F\left(s, u_{s}\right) d s
$$

satisfazendo $u_{\sigma}=\varphi$. Se o conjunto $\left\{u_{t} ; \sigma \leq t<b\right\}$ é limitado então $b=\infty$.

\section{Demonstração}

Suponhamos, por absurdo, que $b<\infty$ e consideremos os conjuntos $\Gamma=\overline{\left\{u_{t} ; \sigma \leq t<b\right\}}$ e $B=\{(t, \psi) ; 0 \leq t \leq b$ e $\psi \in \Gamma\}$

Então $B$ é um conjunto limitado de $\mathbb{R} \times \mathcal{B}$ e $\left(t, u_{t}\right) \in B$ para $\sigma \leq t<b$, o que contradiz o teorema anterior. 
A seguir consideramos $u:(-\infty, \sigma+b] \rightarrow X^{\alpha}$ uma solução da equação integral (1.8) com condição inicial $u_{\sigma}=\varphi$.

Teorema 1.24 Sejam $F: \mathbb{R} \times \mathcal{B} \rightarrow X$ localmente Hölder contínua em $t \in \mathbb{R}$, com expoente $0<\gamma \leq 1$, e localmente Lipschitz contínua em $\varphi \in \mathcal{B} e$ $\varphi:(-\infty, 0] \rightarrow X^{\alpha}$ localmente Hölder contínua com expoente $0<\beta<1-\alpha$. Então a função $t \mapsto u(t)$ é de classe $C^{1}$ em $\sigma<t<\sigma+b$.

\section{Demonstração}

Convém observar que usamos, nesta demonstração, o teorema 1.5.

Como $F$ é localmente Hölder contínua em $t \in \mathbb{R}$ e localmente Lipschitz contínua em $\varphi \in \mathcal{B}$ existem $b_{1}>0, \delta>0$ e $L$ e $D$ constantes positivas tais que

$$
\left\|F\left(t_{1}, \varphi_{1}\right)-F\left(t_{2}, \varphi_{2}\right)\right\|_{X} \leq L\left(\left|t_{1}-t_{2}\right|^{\gamma}+\left\|\varphi_{1}-\varphi_{2}\right\|_{\mathcal{B}}\right)
$$

e

$$
\left\|F\left(t, \varphi_{1}\right)\right\|_{X} \leq D
$$

se $t_{1}, t_{2} \in\left[\sigma, \sigma+b_{1}\right],\left\|\varphi_{1}-\varphi\right\|_{\mathcal{B}} \leq \delta$ e $\left\|\varphi_{2}-\varphi\right\|_{\mathcal{B}} \leq \delta$.

Mostramos, inicialmente, que $u_{t}$ é localmente Hölder contínua em $(\sigma, \sigma+b]$. Para isto, consideremos a função $v:(-\infty, \sigma+b] \rightarrow X^{\alpha}$ definida por

$$
v(t)= \begin{cases}e^{-A(t-\sigma)} \varphi(0), & \text { se } \sigma<t \leq \sigma+b \\ \varphi(t-\sigma), & \text { se } t \leq \sigma\end{cases}
$$

Seja $w(t)=u(t)-v(t)$ para $-\infty<t \leq \sigma+b$. Como $v_{t}$ é Hölder contínua por hipótese, resta-nos mostrar que $w_{t}$ é Hölder contínua em $[\sigma, \sigma+b]$. 
De fato, para $\sigma \leq t<t+h \leq \sigma+b$ temos

$$
\begin{aligned}
& \|w(t+h)-w(t)\|_{X^{\alpha}} \\
& \leq\left\|\int_{\sigma}^{t}\left(e^{-A h}-I\right) e^{-A(t-s)} F\left(s, u_{s}\right) d s\right\|_{X^{\alpha}} \\
& +\left\|\int_{t}^{t+h} e^{-A(t+h-s)} F\left(s, u_{s}\right) d s\right\|_{X^{\alpha}} \\
& \leq \int_{\sigma}^{t} \frac{1}{\beta} N_{1-\beta} h^{\beta}\left\|A^{\beta} A^{\alpha} e^{-A(t-s)} F\left(s, u_{s}\right)\right\|_{X} d s \\
& +N_{\alpha} D \frac{h^{1-\alpha}}{1-\alpha} \\
& \leq \frac{1}{\beta} N_{1-\beta} N_{\alpha+\beta} D h^{\beta} \frac{(t-\sigma)^{1-\alpha-\beta}}{1-\alpha-\beta} \\
& +\frac{N_{\alpha} D}{1-\alpha} h^{1-\alpha} \\
& \leq O\left(h^{\beta}\right) .
\end{aligned}
$$

Observemos agora que $w_{t+h}-w_{t}$ é uma função contínua com suporte compacto contido em $[\sigma-t-h, 0]$ e disto segue, usando o resultado (1.6) (página 37), que

$$
\begin{aligned}
& \left\|w_{t+h}-w_{t}\right\|_{\mathcal{B}} \\
& \leq K(-\sigma+t+h) \max _{\sigma-t-h \leq \theta \leq 0}\|w(t+h+\theta)-w(t+\theta)\|_{X^{\alpha}} \\
& \leq K(-\sigma+t+h) \\
& \quad \max \left\{\max _{\sigma \leq s \leq \sigma+h}\|w(s)\|_{X^{\alpha},} \max _{\sigma+h \leq s \leq t+h}\|w(s)-w(s-h)\|_{X^{\alpha}}\right\} \\
& \leq O\left(h^{\beta}\right),
\end{aligned}
$$

o que implica que $u_{t}$ é localmente Hölder contínua em $(\sigma, \sigma+b]$.

Assim, para $\sigma<t \leq \sigma+b$, temos

$$
\begin{aligned}
\left\|F\left(t+h, u_{t+h}\right)-F\left(t, u_{t}\right)\right\|_{X} & \leq L\left(h^{\gamma}+\left\|u_{t+h}-u_{t}\right\|_{\mathcal{B}}\right) \\
& \leq O\left(h^{\nu}\right)
\end{aligned}
$$


sendo $\nu=\min \{\gamma, \beta\}$, ou seja, $t \mapsto F\left(t, u_{t}\right)$ é localmente Hölder contínua em $(\sigma, \sigma+b]$.

Teorema 1.25 Sejam $F: \mathbb{R} \times \mathcal{B} \rightarrow X$ localmente Hölder contínua em $t \in \mathbb{R}$, com expoente $0<\delta \leq 1$, e localmente Lipschitz contínua em $\varphi \in \mathcal{B} e$ $\varphi:(-\infty, 0] \rightarrow X^{\alpha}$ localmente Hölder contínua com expoente $0<\beta<1-\alpha$. Então qualquer solução $u:(\sigma, \sigma+b] \rightarrow X^{\alpha}$ de $\dot{u}(t)+A u(t)=F\left(t, u_{t}\right)$, com $u_{0}=\varphi$, é tal que $\frac{d u(t)}{d t} \in X^{\gamma}$ para todo $0 \leq \gamma<\nu, \operatorname{com} \nu=\min \{\delta, 1-\alpha\} e$ $\left\|\frac{d u(t)}{d t}\right\|_{X^{\gamma}}=O\left((t-\sigma)^{\alpha-\gamma-1}\right)$ quando $t \rightarrow \sigma$.

Como a demonstração deste teorema é muito semelhante à demonstração do teorema 1.15, será omitida neste caso. 


\section{Capítulo 2}

\section{Problema contínuo}

Nosso objetivo, neste capítulo, é mostrar existência e unicidade global de soluções da equação

$$
\begin{cases}u_{t}(x, t)=a u_{x x}(x, t)+u(x, t)(1-u(x, t-r)), & \text { se } 0<x<1, t>0, \\ u_{x}(0, t)=u_{x}(1, t)=0, & \text { se } t \geq 0, \\ u(x, t)=\varphi(x, t)>0, & \text { se } 0<x<1, t \in[-r, 0]\end{cases}
$$

Para isto usamos teoria de semigrupo, ou seja, tratamos (2.1) como uma equação de evolução em um espaço de Banach $X$ e usamos os resultados abstratos do capítulo 1. Além disso, mostramos que o problema acima gera semigrupo, o qual é limitado dissipativo, e possui atrator global.

Cabe observar que precisamos do primeiro autovalor positivo para termos o semigrupo linear dissipativo o que permite-nos concluir que a solução é limitada e o semigrupo limitado dissipativo. Assim, consideramos a seguir, o problema

$$
\left\{\begin{array}{lr}
u_{t}(x, t)=a u_{x x}(x, t)-u(x, t)+u(x, t)(1-u(x, t-r))+u(x, t), \\
u_{x}(0, t)=u_{x}(1, t)=0, & \text { se } 0<x<1, t>0 \\
u(x, t)=\varphi(x, t)>0, & \text { se } t \geq 0
\end{array}\right.
$$




\subsection{Existência e unicidade de soluções}

Com o propósito de estudarmos existência e unicidade global de soluções da equação (2.2) consideramos o espaço $X=L^{2}(0,1), A$ um operador em $X$ associado à parte linear e às condições de fronteira de (2.2)

$$
\begin{aligned}
A: D(A) \subset L^{2}(0,1) & \rightarrow L^{2}(0,1) \\
\phi & \mapsto-a \phi^{\prime \prime}
\end{aligned}
$$

$\operatorname{com} D(A)=\left\{\phi \in H^{2}(0,1) ; \phi(t)>0, \phi^{\prime}(0)=\phi^{\prime}(1)=0\right\} \mathrm{e}$

$$
\begin{aligned}
F: C\left([-r, 0], H^{1}(0,1)\right) & \rightarrow L^{2}(0,1) \\
\varphi & \mapsto F(\varphi)(x)=\varphi(0)(x)(2-\varphi(-r)(x)),
\end{aligned}
$$

associada à parte não linear de (2.2).

Então a equação (2.2) pode ser, pelo menos formalmente, escrita como

$$
\begin{cases}\dot{u}(t)+(A+I) u(t)=F\left(u_{t}\right), & \text { se } t>0, \\ u(t)=\varphi(t)>0, & \text { se }-r \leq t \leq 0\end{cases}
$$

com $u_{t} \in C\left([-r, 0], H^{1}(0,1)\right)$ definida por $u_{t}(\theta)=u(t+\theta)$.

Julgamos importante observar que vamos considerar em $H^{1}(0,1)$ somente as funções positivas e, por simplicidade, continuamos denotando este espaço por $H^{1}(0,1)$.

A proposição abaixo, juntamente com os teoremas 1.6 e 1.14 , nos garantem existência e unicidade de soluções de (2.3).

Proposição 2.1 A função $F: C\left([-r, 0], H^{1}(0,1)\right) \rightarrow L^{2}(0,1)$ definida por $F(\varphi)(x)=\varphi(0)(x)(2-\varphi(-r)(x))$ é localmente Lipschitz contínua. 


\section{Demonstração}

Sejam $\phi, \psi \in C\left([-r, 0], H^{1}(0,1)\right)$ tais que $\|\phi\|_{C\left([-r, 0], H^{1}(0,1)\right)}<\rho$ e $\|\psi\|_{C\left([-r, 0], H^{1}(0,1)\right)}<\rho$ para algum $\rho>0$.

Então,

$$
\begin{aligned}
& \|F(\phi)-F(\psi)\|_{L^{2}}^{2} \\
& =\quad \int_{0}^{1}|F(\phi)(x)-F(\psi)(x)|^{2} d x \\
& =\quad \int_{0}^{1}|\phi(0)(x)[2-\phi(-r)(x)]-\psi(0)(x)[2-\psi(-r)(x)]|^{2} d x \\
& =\quad \int_{0}^{1} \mid 2(\phi(0)(x)-\psi(0)(x)) \\
& +\quad \psi(0)(x) \psi(-r)(x)-\left.\phi(0)(x) \phi(-r)(x)\right|^{2} d x \\
& \leq \quad 8 \int_{0}^{1}|\phi(0)(x)-\psi(0)(x)|^{2} d x \\
& +\quad 2 \int_{0}^{1} \mid \psi(0)(x) \psi(-r)(x)-\phi(0)(x) \phi(-r)(x) \\
& +\quad \psi(0)(x) \phi(-r)(x)-\left.\psi(0)(x) \phi(-r)(x)\right|^{2} d x \\
& \leq \quad 8\|\phi(0)-\psi(0)\|_{L^{2}}^{2} \\
& +\quad 4 \int_{0}^{1}|\psi(0)(x)|^{2}|\psi(-r)(x)-\phi(-r)(x)|^{2} d x \\
& +\quad 4 \int_{0}^{1}|\phi(-r)(x)|^{2}|\psi(0)(x)-\phi(0)(x)|^{2} d x \\
& \leq \quad 8\|\phi(0)-\psi(0)\|_{H^{1}}^{2} \\
& \stackrel{H^{1}(0,1) \hookrightarrow C(0,1)}{+} 8\|\psi(0)\|_{H^{1}}^{2}\|\psi(-r)-\phi(-r)\|_{L^{2}}^{2} \\
& +\quad 8\|\phi(-r)\|_{H^{1}}^{2}\|\psi(0)-\phi(0)\|_{L^{2}}^{2} \\
& \leq \quad 8\|\phi-\psi\|_{C\left([-r, 0], H^{1}(0,1)\right)}^{2} \\
& +\quad 8 \rho^{2}\|\phi-\psi\|_{C\left([-r, 0], H^{1}(0,1)\right)}^{2}+8 \rho^{2}\|\phi-\psi\|_{C\left([-r, 0], H^{1}(0,1)\right)}^{2} \\
& =\quad\left(16 \rho^{2}+8\right)\|\phi-\psi\|_{C\left([-r, 0], H^{1}(0,1)\right)}^{2} .
\end{aligned}
$$


O teorema 1.11, juntamente com a proposição (2.1) e a proposição (2.2) abaixo, nos garantem existência global de soluções de (2.3).

Proposição 2.2 Seja $B \subset C\left([-r, 0], H^{1}(0,1)\right)$ um conjunto limitado com $\phi \in B$ positiva. Então $F(B) \subset L^{2}(0,1)$ é limitado.

\section{Demonstração}

Sejam $B \subset C\left([-r, 0], H^{1}(0,1)\right)$ um conjunto limitado e $\rho>0$ tal que para todo $\phi \in B,\|\phi\|_{C\left([-r, 0], H^{1}(0,1)\right)}<\rho$ e $\max _{|x|,|y| \leq \sqrt{2} \rho}|f(x, y)|=M$, sendo $f(x, y)=x(2-y)$.

Então,

$$
\begin{aligned}
& \|F(\phi)\|_{L^{2}}^{2} \\
& =\int_{0}^{1}|F(\phi)(x)|^{2} d x \\
& =\int_{0}^{1}|f(\phi(0)(x), \phi(-r)(x))|^{2} d x \\
& =M^{2}
\end{aligned}
$$

pois $H^{1}(0,1)$ está continuamente imerso em $C(0,1)$.

\subsection{Atrator global}

Vamos agora discutir a existência de atrator global para o problema (2.2). Boa parte desta seção deve-se a Hale [8].

Definição 2.3 Sejam $X$ um espaço de Banach e $T(t): X \rightarrow X, t \geq 0$, um semigrupo. 
(i) Um conjunto $B \subset X$ atrai um conjunto $C \subset X$ através de $T(t)$ se $\operatorname{dist}(T(t) C, B) \rightarrow 0$ quando $t \rightarrow \infty$, sendo $\operatorname{dist}(A, B)=\sup _{y \in A} \inf _{x \in B} d(y, x)$, $A, B$ subconjuntos de $X$.

(ii) O semigrupo $T(t)$ é assintoticamente liso se para cada conjunto $B \subset X$ não vazio, fechado e limitado para o qual $T(t) B \subset B$, para todo $t \geq 0$, existe um conjunto compacto $J \subset B$ que atrai $B$.

(iii) O semigrupo $T(t)$ é ponto (limitado) dissipativo se existe um conjunto limitado $B \subset X$ que atrai cada ponto (subconjunto limitado) de $X$ através de $T(t)$.

Definição 2.4 Um conjunto compacto invariante maximal para um semigrupo $T(t): X \rightarrow X, t \geq 0, X$ espaço de Banach, é chamado atrator global para $T(t)$ se atrai cada subconjunto limitado de $X$.

Teorema 2.5 Se um semigrupo $T(t): X \rightarrow X, t \geq 0, X$ espaço de Banach, é assintoticamente liso, ponto dissipativo e órbitas de conjuntos limitados são limitadas então $T(t)$ possui atrator global.

Proposição 2.6 Um semigrupo $T(t): X \rightarrow X, t \geq 0, X$ espaço de Banach, que é condicionalmente completamente contínuo para $t \geq t_{0}$ é assintoticamente liso.

Lembremos que as soluções do problema (2.2) definem um semigrupo $U(t)$ fortemente continuo e condicionalmente completamente contínuo para 
cada $t>r$ fixado (proposição 1.17) e, por regularização das soluções, segue que $U(t)$ é compacto. E para concluirmos que órbitas de conjuntos limitados são limitadas necessitamos dos lemas abaixo, cujas demonstrações podem ser encontradas em Luckhaus [19] ou Wu [31]. Observemos que $\frac{\partial}{\partial \eta}$ é a derivada normal exterior ao intervalo $(0,1)$.

Lema 2.7 Seja $p \geq 2$ e suponhamos que existam $T>r>0$ e $\alpha \in(0,1)$ tal que qualquer solução clássica de

$$
\begin{cases}\frac{\partial v(x, t)}{\partial t} \leq \mu \Delta v(x, t)+v(x, t), & \text { se } \quad(x, t) \in(0,1) \times(0, T), \\ v(x, t) v(x, t-r)=0, & \text { se } \quad(x, t) \in(0,1) \times(r, T), \\ \frac{\partial v(x, t)}{\partial \eta}=0, & \text { se } \quad(x, t) \in(\{0\} \cup\{1\}) \times(0, T) \\ v(x, t) \geq 0, & \text { se }(x, t) \in(0,1) \times(0, T)\end{cases}
$$

satisfaz a estimativa de decaimento

$$
\int_{0}^{1} v^{p}(x, T) d x \leq \alpha \int_{0}^{1} v^{p}(x, 0) d x
$$

Então existe uma constante $K=K(f,(0,1), \alpha, T)$, que independe da condição inicial, tal que a solução clássica de

$$
\begin{aligned}
& \left\{\begin{array}{lr}
\frac{\partial u(x, t)}{\partial t}=\mu \Delta u(x, t)+f(u(x, t), u(x, t-r)), & \text { se } x \in(0,1), t>0, \\
\frac{\partial u(x, t)}{\partial \eta}=0, & \text { se } x \in(\{0\} \cup\{1\}), t>0, \\
u(x, t) \geq 0, & \text { se } x \in(0,1), t>0,
\end{array}\right. \\
& \text { com } \int_{-r}^{0} \int_{0}^{1} u^{p}(x, t) d x d t<\infty \text { satisfaz } \lim _{t \rightarrow \infty} \sup \int_{0}^{1} u^{p}(x, t) d x \leq K .
\end{aligned}
$$

Lema 2.8 Seja $v$ uma solução de (2.4) $\operatorname{com} T>r>0$, então

$$
\int_{0}^{1} v^{p}(x, T) d x \leq\left[e^{\left(2 T\left(1-\frac{\pi^{2} r^{2}}{4 r^{2}}\right)\right)}\right] \int_{0}^{1} v^{p}(x, 0) d x
$$


Lembremos que $\left\|e^{-A t}\right\|_{L(X)} \leq M$ para todo $t \geq 0$, como observado no capítulo 1, e $N_{\alpha}$ é dado pelo teorema 1.5. E seja $\lambda>0$ o primeiro autovalor de $A+I$.

Teorema 2.9 Seja $U(t), t \geq 0$, o semigrupo fortemente continuo associado às soluções da equação (2.2). Então $\gamma^{+}(B)=\bigcup_{t \geq 0} U(t) B$ é limitado se $B$ é limitado.

\section{Demonstração}

Temos, pelos lemas 2.7 e 2.8 , que existem $t_{0}>r$ e uma constante $K_{1}$, que independe da condição inicial, tal que $\|u(t)\|_{L^{p}} \leq K_{1}$, para todo $t \geq t_{0}$

Observemos que existe uma constante $K_{2}$ tal que $\|u(t)\|_{L^{p}} \leq K_{2}$, para todo $-r \leq t \leq t_{0}$, pois órbitas de conjuntos limitados são limitadas em tempo finito.

Assim, temos que

$$
\begin{aligned}
& \|u(t)\|_{H^{1}} \\
& \leq\left\|e^{-(A+I) t} \varphi(0)\right\|_{H^{1}}+\int_{0}^{t}\left\|e^{-(A+I)(t-s)} F\left(u_{s}\right)\right\|_{H^{1}} d s \\
& \leq M\|\varphi(0)\|_{H^{1}} \\
& +2 N_{1 / 2}\left[\int_{0}^{t_{0}} e^{-\lambda(t-s)}(t-s)^{-1 / 2}\|u(s)\|_{L^{2}} d s\right. \\
& \left.+\int_{t_{0}}^{t} e^{-\lambda(t-s)}(t-s)^{-1 / 2}\|u(s)\|_{L^{2}} d s\right] \\
& +N_{1 / 2}\left[\int_{0}^{t_{0}} e^{-\lambda(t-s)}(t-s)^{-1 / 2}\|u(s)\|_{L^{4}}\|u(s-r)\|_{L^{4}} d s\right. \\
& +\int_{t_{0}}^{t_{0}+r} e^{-\lambda(t-s)}(t-s)^{-1 / 2}\|u(s)\|_{L^{4}}\|u(s-r)\|_{L^{4}} d s \\
& \left.+\int_{t_{0}+r}^{t} e^{-\lambda(t-s)}(t-s)^{-1 / 2}\|u(s)\|_{L^{4}}\|u(s-r)\|_{L^{4}} d s\right]
\end{aligned}
$$




$$
\begin{aligned}
& \leq M\|\varphi(0)\|_{H^{1}} \\
& +N_{1 / 2}\left(2 K+K^{2}\right) \int_{0}^{t} e^{-\lambda(t-s)}(t-s)^{-1 / 2} d s
\end{aligned}
$$

sendo $K=\max \left\{K_{1}, K_{2}\right\}$.

Assim, $\left\|u_{t}\right\|_{C_{\alpha}}$ é limitada para todo $t \geq 0$.

Como acabamos de mostrar, órbitas de conjuntos limitados são limitadas para o problema (2.2). Assim, os resultados até aqui apresentados e a proposição a seguir, nos garantem a existência de atrator global para o problema (2.2).

Proposição 2.10 Seja $U(t), t \geq 0$, o semigrupo fortemente contínuo associado às soluções da equação (2.2). Então $U(t)$ é limitado dissipativo.

\section{Demonstração}

Temos, pelos lemas 2.7 e 2.8 , que existem $t_{0}>r$ e uma constante $K_{1}$, que independe da condição inicial, tal que $\|u(t)\|_{L^{p}} \leq K_{1}$, para todo $t \geq t_{0}$

Observemos que existe uma constante $K_{2}$ tal que $\|u(t)\|_{H^{1}} \leq K_{2}$, para todo $-r \leq t \leq t_{0}$, pois órbitas de conjuntos limitados são limitadas.

Seja $t_{1}>t_{0}$ tal que $M e^{-\lambda\left(t_{1}-t_{0}\right)} K_{2}<1$.

Assim, temos que

$$
\begin{aligned}
& \|u(t)\|_{H^{1}} \\
& =\left\|e^{-(A+I)\left(t-t_{0}\right)} u\left(t_{0}\right)+\int_{t_{0}}^{t} e^{-(A+I)(t-s)} F\left(u_{s}\right) d s\right\|_{H^{1}} \\
& \leq M e^{-\lambda\left(t-t_{0}\right)}\left\|u\left(t_{0}\right)\right\|_{H^{1}} \\
& \quad+2 N_{1 / 2} \int_{t_{0}}^{t} e^{-\lambda(t-s)}(t-s)^{-1 / 2}\|u(s)\|_{L^{2}} d s
\end{aligned}
$$




$$
\begin{aligned}
& +N_{1 / 2}\left[\int_{t_{0}}^{t_{0}+r} e^{-\lambda(t-s)}(t-s)^{-1 / 2}\|u(s)\|_{L^{4}}\|u(s-r)\|_{L^{4}} d s\right. \\
& \left.+\int_{t_{0}+r}^{t} e^{-\lambda(t-s)}(t-s)^{-1 / 2}\|u(s)\|_{L^{4}}\|u(s-r)\|_{L^{4}} d s\right] \\
& \leq M e^{-\lambda\left(t-t_{0}\right)}\left\|u\left(t_{0}\right)\right\|_{H^{1}} \\
& +N_{1 / 2}\left(2 K+K^{2}\right) \int_{t_{0}}^{t} e^{-\lambda(t-s)}(t-s)^{-1 / 2} d s \\
& \leq 1+N_{1 / 2}\left(2 K+K^{2}\right) \int_{t_{0}}^{t} e^{-\lambda(t-s)}(t-s)^{-1 / 2} d s
\end{aligned}
$$

para $t \geq t_{1}$ e $K=\max \left\{K_{1}, K_{2}\right\}$

É importante observarmos que, como estamos interessados em estudar as soluções do problema (2.2) somente em uma vizinhança do atrator, podemos cortar a não linearidade $f: \mathbb{R}^{2} \rightarrow \mathbb{R}$ definida por $f(x, y)=x(2-y)$, obtendo uma nova função $g: \mathbb{R}^{2} \rightarrow \mathbb{R}$ de tal forma que $g$ e suas derivadas até segunda ordem sejam limitadas. E o novo sistema, definido a partir da função $g$,

$$
\begin{cases}u_{t}(x, t)=a u_{x x}(x, t)-u(x, t)+g(u(x, t), u(x, t-r)) \\ u_{x}(0, t)=u_{x}(1, t)=0, & \text { se } 0<x<1, t>0, \\ u(x, t)=\varphi(x, t)>0, & \text { se } t \geq 0, \\ & \text { se } 0<x<1, t \in[-r, 0] .\end{cases}
$$

mantém o mesmo atrator que o problema (2.2) e as mesmas soluções em uma vizinhança do atrator. 


\section{Capítulo 3}

\section{Problema discretizado}

Como já dissemos, neste trabalho procuramos comparar a dinâmica assintótica do problema parabólico escalar com retardamento (2.1)

$$
\begin{cases}u_{t}(x, t)=a u_{x x}(x, t)+u(x, t)(1-u(x, t-r)), & \text { se } 0<x<1, t>0, \\ u_{x}(0, t)=u_{x}(1, t)=0, & \text { se } t \geq 0, \\ u(x, t)=\varphi(x, t)>0, & \text { se } 0<x<1, t \in[-r, 0]\end{cases}
$$

e sua respectiva discretização. No capítulo 2 mostramos existência e unicidade de soluções do problema dado; mostramos ainda a existência de atrator global.

Neste capítulo apresentamos uma discretização do retardo e estimamos a proximidade das soluções dos problemas contínuo e discretizado. Para isto, consideramos um problema com retardo infinito e mostramos que suas soluções convergem às soluções do problema inicial, como nos afirma o teorema 3.13 , e a seguir que o problema com retardo infinito e o problema discretizado são equivalentes, proposição 3.14 .

Além disso, apresentamos uma discretização espacial e obtemos, em cada caso, uma variedade invariante exponencialmente atratora e comparamos os campos vetoriais, dos problemas contínuo e discretizado, sobre a variedade invariante, como será discutido na seção 3.3 . 


\subsection{Discretização do retardo}

A discretização do retardo apresentada nesta seção é feita para o problema (2.5)

$$
\begin{cases}u_{t}(x, t)=a u_{x x}(x, t)-u(x, t)+g(u(x, t), u(x, t-r)) \\ u_{x}(0, t)=u_{x}(1, t)=0, & \text { se } 0<x<1, t>0, \\ u(x, t)=\varphi(x, t)>0, & \text { se } t \geq 0, \\ & \text { se } 0<x<1, t \in[-r, 0]\end{cases}
$$

lembrando que os problemas (2.1) e (2.5) possuem o mesmo atrator e as mesmas soluções em uma vizinhança do atrator.

Boa parte desta seção é inspirada nos trabalhos de Gedeon e Hines [7] e Hines $[15,16]$, porém, até onde conhecemos, uma discretização desse tipo nunca foi feita para equações funcionais parciais.

Com o propósito de simplificarmos os cálculos vamos reescalonar o tempo em (2.5) a fim de obtermos um problema com retardo unitário.

Para isto seja $y(x, t)=u(x, t r)$. Então $y(x, t)$ satisfaz a equação diferencial

$$
\begin{cases}y_{t}(x, t)=\operatorname{ar} y_{x x}(x, t)-r y(x, t)+r g(y(x, t), & y(x, t-1)) \\ y_{x}(0, t)=y_{x}(1, t)=0, & \text { se } 0<x<1, t>0, \\ y(x, t)=\varphi(x, t r)>0, & \text { se } t \geq 0\end{cases}
$$

Consideremos agora a discretização do retardo de (3.1), isto é, dividimos o intervalo $[-1,0]$ em $n$ subintervalos iguais, usamos a aproximação linear da condição inicial em cada subintervalo e consideramos $U_{k}(x, t)$ a aproximação de $y\left(x, t-\frac{k}{n}\right), k=0, \ldots, n$.

Assim, podemos aproximar (3.1) por um sistema formado por uma 
equação diferencial parcial e um sistema de equações diferenciais ordinárias,

$$
\left\{\begin{array}{l}
\left(U_{0}(x, t)\right)_{t}=\operatorname{ar}\left(U_{0}(x, t)\right)_{x x}-r U_{0}(x, t)+r g\left(U_{0}(x, t), U_{n}(x, t)\right) \\
\left(U_{1}(x, t)\right)_{t}=n\left(U_{0}(x, t)-U_{1}(x, t)\right) \\
\vdots \\
\left(U_{n}(x, t)\right)_{t}=n\left(U_{n-1}(x, t)-U_{n}(x, t)\right)
\end{array}\right.
$$

para $0<x<1$ e $t>0$, com condições de fronteira

$$
\left(U_{0}\right)_{x}(0, t)=\left(U_{0}\right)_{x}(1, t)=0, t \geq 0
$$

e condições iniciais

$$
\left\{\begin{array}{l}
U_{0}(x, 0)=\varphi(x, 0) \\
U_{1}(x, 0)=\varphi\left(x, \frac{-r}{n}\right) \\
\vdots \\
U_{n}(x, 0)=\varphi(x,-r)
\end{array}\right.
$$

para $0<x<1$

Com o propósito de estudarmos existência e unicidade de soluções do problema (3.2) consideramos $A$ um operador em $L^{2}(0,1)$ definido por

$$
\begin{aligned}
A: D(A) \subset L^{2}(0,1) & \rightarrow L^{2}(0,1) \\
\phi & \mapsto-a r \phi^{\prime \prime},
\end{aligned}
$$

$\operatorname{com} D(A)=\left\{\phi \in H^{2}(0,1) ; \phi(t)>0, \phi^{\prime}(0)=\phi^{\prime}(1)=0\right\}$

$$
\begin{aligned}
G: H^{1}(0,1) \times\left(H^{1}(0,1)\right)^{n} & \rightarrow L^{2}(0,1) \\
(\varphi, \psi) & \mapsto G(\varphi, \psi)(x)=\operatorname{rg}\left(\varphi(x), \psi_{n}(x)\right)
\end{aligned}
$$

e $F: H^{1}(0,1) \times\left(H^{1}(0,1)\right)^{n} \rightarrow\left(L^{2}(0,1)\right)^{n}$ definida por

$$
(F(\varphi, \psi))_{i}(x)= \begin{cases}n\left(\varphi(x)-\psi_{1}(x)\right), & \text { se } i=1 \\ n\left(\psi_{i-1}(x)-\psi_{i}(x)\right), & \text { se } 2 \leq i \leq n\end{cases}
$$

Então a equação (3.2) pode ser, pelo menos formalmente, escrita como

$$
\left\{\begin{array}{l}
\dot{U}_{0}(t)+(A+r I) U_{0}(t)=G\left(U_{0}, V\right) \\
\dot{V}(t)=F\left(U_{0}, V\right)
\end{array}\right.
$$


$\operatorname{com} V=\left(U_{1}, \ldots, U_{n}\right)$.

Sabemos que $A$ é um operador setorial e $F$ e $G$ são Lipschitz contínuas, pois $F$ é linear e $g$ possui derivada limitada.

A proposição abaixo, que pode ser encontrada em Henry [13], juntamente com as observações acima, nos garantem existência e unicidade de soluções de (3.2).

Proposição 3.1 Sejam $A: D(A) \subset X \rightarrow X$ um operador setorial, $U$ um conjunto aberto em $\mathbb{R} \times X^{\alpha} \times Y, f: U \rightarrow X$ e $g: U \rightarrow Y$ localmente Lipschitz contínuas. Então o sistema

$$
\left\{\begin{array}{l}
\dot{x}+A x=f(t, x, y) \\
\dot{y}=g(t, x, y)
\end{array}\right.
$$

possui uma única solução.

E como a função $g$ é de classe $C^{2}$, com derivadas limitadas, temos que as soluções de (3.3) existem para todo $t>0$ e, além disso, como órbitas de conjuntos limitados são limitadas, o problema (3.3) possui atrator global.

\subsection{Proximidade das soluções do problema con- tínuo e discretizado}

Vamos agora mostrar a proximidade das soluções do problema contínuo e discretizado. Antes de abordarmos esta questão apresentamos alguns resultados preliminares $[5,7,20]$. 
Definição 3.2 Uma função $f: \mathbb{R} \rightarrow \mathbb{R}$ é seccionalmente contínua se, dados $a<b$, existem $a \leq a_{1}<\cdots<a_{n} \leq b$ tais que $f$ é contínua em cada intervalo aberto $\left(a_{j}, a_{j+1}\right), j=1, \ldots, n-1$ e existem os limites $\lim _{x \rightarrow\left(a_{j}\right)_{+}} f(x)$ $e \lim _{x \rightarrow\left(a_{j}\right)_{-}} f(x)$

Definição 3.3 Uma seqüência de funções $k_{n}:(-\infty, 0] \rightarrow[0, \infty)$ seccionalmente continuas é uma seqüência de núcleos de Dirac se satisfaz as propriedades abaixo para algum $T>0$,

(d1) $k_{n}(s) \geq 0$

(d2) $\int_{-\infty}^{0} k_{n}(s) d s=1$

(d3) dados $\varepsilon>0$ e $\delta>0$, existe $n_{0}$ tal que $\int_{|s-(-T)|>\delta} k_{n}(s) d s<\varepsilon$ para $n \geq n_{0}$

Definição 3.4 Uma função $g: \mathbb{R} \rightarrow \mathbb{R}$ é uma função densidade de probabilidade se

(i) $g(x) \geq 0$, para todo $x \in \mathbb{R}$;

(ii) $\int_{-\infty}^{\infty} g(x) d x=1$.

Definição 3.5 A média da função densidade de probabilidade g é definida por

$$
\mu_{x}=\int_{-\infty}^{\infty} x g(x) d x
$$


Definição 3.6 A variância da função densidade de probabilidade $g$ é definida por

$$
\sigma_{x}^{2}=\int_{-\infty}^{\infty}\left(x-\mu_{x}\right)^{2} g(x) d x,
$$

sendo $\mu_{x}$ a média da função densidade de probabilidade $g$.

A seguir, mostramos que a seqüência $Q_{n}(s)=n^{n} \frac{(-s)^{n-1}}{(n-1) !} e^{n s}, s \leq 0$ e $n \in \mathbb{N}$ é uma seqüência de núcleos de Dirac. Para isto, mostramos que $r_{k}^{n}:(-\infty, 0] \rightarrow \mathbb{R}$, definida por $r_{k}^{n}(s)=n^{k} \frac{(-s)^{k-1}}{(k-1) !} e^{n s}$, é uma função densidade de probabilidade para todo $k=1, \ldots, n$ e usamos a desigualdade de Chebyshev [20]

$$
\int_{-\infty}^{\mu_{k}^{n}-\nu} r_{k}^{n}(s) d s+\int_{\mu_{k}^{n}+\nu}^{0} r_{k}^{n}(s) d s \leq \frac{\left(\sigma_{k}^{n}\right)^{2}}{\nu^{2}}, \text { para todo } \nu>0,
$$

com $\mu_{k}^{n}$ a média de $r_{k}^{n}$ e $\left(\sigma_{k}^{n}\right)^{2}$ a variância.

Lema 3.7 A função $r_{k}^{n}:(-\infty, 0] \rightarrow \mathbb{R}$ definida por $r_{k}^{n}(s)=n^{k} \frac{(-s)^{k-1}}{(k-1) !} e^{n s} e^{e}$ uma função densidade de probabilidade para todo $k=1, \ldots, n$.

\section{Demonstração}

Temos que $r_{k}^{n}(s) \geq 0$, para todo $s \leq 0$. Além disso,

$$
\begin{aligned}
\int_{-\infty}^{0} r_{k}^{n}(s) d s & =\frac{n^{k}}{(k-1) !} \int_{-\infty}^{0}(-s)^{k-1} e^{n s} d s \\
& =\frac{n^{k}}{(k-1) !} \int_{0}^{\infty} s^{k-1} e^{-n s} d s \\
& =1 .
\end{aligned}
$$

Lema 3.8 Seja $\mu_{k}^{n}$ a média da função $r_{k}^{n}$. Então $\mu_{k}^{n}=-\frac{k}{n}$ para todo $k=1, \ldots, n$. 


\section{Demonstração}

Demonstramos este lema por indução. Fazendo $k=1$ temos

$$
\begin{aligned}
\mu_{1}^{n} & =\int_{-\infty}^{0} s n e^{n s} d s \\
& =\lim _{b \rightarrow-\infty}\left[-b e^{n b}-\frac{1}{n}+\frac{1}{n} e^{n b}\right] \\
& =\lim _{b \rightarrow-\infty}\left[\frac{-b}{1 / e^{n b}}\right]-\frac{1}{n} \\
& =\lim _{b \rightarrow-\infty}\left[\frac{e^{n b}}{n}\right]-\frac{1}{n} \\
& =-\frac{1}{n} .
\end{aligned}
$$

Suponhamos que a igualdade seja válida para $k$, isto é,

$$
\mu_{k}^{n}=\int_{-\infty}^{0} s n^{k} \frac{(-s)^{k-1}}{(k-1) !} e^{n s} d s=-\frac{k}{n}
$$

Assim, para $k+1$, temos

$$
\begin{aligned}
\mu_{k+1}^{n} & =\int_{-\infty}^{0} s n^{k+1} \frac{(-s)^{k}}{k !} e^{n s} d s \\
& =\frac{n^{k+1}}{k !}(-1)^{k} \int_{-\infty}^{0} s^{k+1} e^{n s} d s \\
& =\frac{n^{k+1}}{k !}(-1)^{k+1} \int_{-\infty}^{0}(k+1) s^{k} \frac{e^{n s}}{n} d s \\
& =\frac{k+1}{k} \int_{-\infty}^{0} s n^{k} \frac{(-s)^{k-1}}{(k-1) !} e^{n s} d s \\
& =\frac{k+1}{k}\left(-\frac{k}{n}\right) \\
& =-\frac{k+1}{n} .
\end{aligned}
$$

Lema 3.9 Seja $\left(\sigma_{k}^{n}\right)^{2}$ a variância da função $r_{k}^{n}$. Então $\left(\sigma_{k}^{n}\right)^{2}=\frac{k}{n^{2}}$ para todo $k=1, \ldots, n$. 


\section{Demonstração}

Segue, da definição 3.6, que $\left(\sigma_{k}^{n}\right)^{2}=\int_{-\infty}^{0} s^{2} r_{k}^{n}(s) d s-\left(\mu_{k}^{n}\right)^{2}$.

E como

$$
\begin{aligned}
\int_{-\infty}^{0} s^{2} r_{k}^{n} d s & =\int_{-\infty}^{0} \frac{n^{k}}{(k-1) !}(-s)^{k+1} e^{n s} d s \\
& =-\left(\frac{k+1}{n}\right) \int_{-\infty}^{0} s \frac{n^{k}}{(k-1) !}(-s)^{k-1} e^{n s} d s \\
& =-\left(\frac{k+1}{n}\right) \mu_{k}^{n} \\
& =\frac{k^{2}+k}{n^{2}}
\end{aligned}
$$

temos que

$$
\begin{aligned}
\left(\sigma_{k}^{n}\right)^{2} & =\frac{k^{2}+k}{n^{2}}-\frac{k^{2}}{n^{2}} \\
& =\frac{k}{n^{2}}
\end{aligned}
$$

Proposição 3.10 A seqüência $r_{k}^{n}(s)=n^{k} \frac{(-s)^{k-1}}{(k-1) !} e^{n s}, s \leq 0$, é uma seqüência de núcleos de Dirac para todo $k=1, \ldots, n$.

\section{Demonstração}

As condições $(d 1)$ e $(d 2)$ foram provadas no lema 3.7. Basta-nos, então, provar a condição $(d 3)$.

Dados $\varepsilon>0$ e $\delta>0$ seja $n_{0}=\frac{1}{\delta^{2} \varepsilon}$. Então, usando a desigualdade de Chebyshev, temos que

$$
\begin{aligned}
\int_{|s-(-k / n)|>\delta} r_{k}^{n}(s) d s & =\int_{-\infty}^{(-k / n)-\delta} r_{k}^{n}(s) d s+\int_{(-k / n)+\delta}^{0} r_{k}^{n}(s) d s \\
& \leq \frac{k / n^{2}}{\delta^{2}} \\
& \leq \frac{1}{n \delta^{2}} \\
& \leq \varepsilon
\end{aligned}
$$


para todo $n \geq n_{0}$.

Consideremos agora o problema com retardo infinito

$\left\{\begin{array}{l}v_{t}(x, t)=\operatorname{ar} v_{x x}(x, t)-r v(x, t)+r g\left(v(x, t), \int_{-\infty}^{t} v(x, s) Q_{n}(s-t) d s\right) \\ v_{0}(x, t)=\hat{\varphi}(x, t) \in C\left((-\infty, 0], H^{1}(0,1)\right)\end{array}\right.$

com

$$
\hat{\varphi}(x, t)= \begin{cases}\varphi(x, r t), & \text { se }-1 \leq t \leq 0 \\ \varphi(x,-r), & \text { se } t \leq-1\end{cases}
$$

e

$$
Q_{n}(s)=n^{n} \frac{(-s)^{n-1}}{(n-1) !} e^{n s}, s \leq 0
$$

Uma questão natural é sobre a escolha do espaço de fase para os problemas (3.1), (3.2) e (3.4), que deve ser escolhido apropriadamente para cada problema. Um exemplo de espaço de fase para o problema (3.4) é

$$
\vec{B}=\left\{\phi:(-\infty, 0] \rightarrow H^{1}(0,1) ; \phi \text { é mensurárel e positiva }\right\}
$$

com a norma

$$
\|\phi\|_{\bar{B}}=\|\phi(0)\|_{H^{1}}+\int_{-\infty}^{0}\|\phi(s)\|_{H^{1}} Q_{1}(s) d s
$$

Este espaço satisfaz os axiomas exigidos na seção 1.2, mas como queremos também incluir o problema (3.1), cujo espaço de fase natural é $C\left([-1,0], H^{1}(0,1)\right)$, precisamos de um espaço no qual a norma incorpora as principais propriedades de ambas as normas $\|\cdot\|_{\bar{B}}$ e $\|\cdot\|_{C\left([-1,0], H^{1}(0,1)\right)}$ e uma escolha seria

$$
B=\left\{\phi:(-\infty, 0] \rightarrow H^{1}(0,1) ; \phi \text { é contínua em }[-1,0] \text { e positiva }\right\}
$$

com a norma

$$
\|\phi\|_{B}=\sup _{-1 \leq s \leq 0}\|\phi(s)\|_{H^{1}}+\int_{-\infty}^{0}\|\phi(s)\|_{H^{1}} Q_{1}(s) d s
$$


para maiores detalhes veja Hines [16] e Henriquez [12].

Mas, infelizmente, não temos convergência da parte não linear na norma acima, e assim uma possibilidade, apontada por Hines [16], é considerarmos os problemas (3.1), (3.2) e (3.4) no espaço

$$
\mathcal{B}=\left\{\phi:(-\infty, 0] \rightarrow H^{1}(0,1) ; \phi \text { é contínua em }[-2,0] \text { e positiva }\right\}
$$

com a norma

$$
\|\phi\|_{\mathcal{B}}=\sup _{-2 \leq s \leq 0}\|\phi(s)\|_{H^{1}}+\int_{-\infty}^{0}\|\phi(s)\|_{H^{1}} Q_{1}(s) d s
$$

Julgamos importante observar que como a função $g$ é de classe $C^{2}$, com derivadas limitadas, as soluções de (3.4) existem para todo $t>0$ e, além disso, como órbitas de conjuntos limitados são limitadas, o problema (3.4) possui atrator global.

A fim de mostrarmos a proximidade das soluções dos problemas com retardo finito e infinito demonstramos o seguinte lema.

Lema 3.11 Sejam $g^{n}: \mathcal{B} \rightarrow L^{2}(0,1)$ definida por

$$
g^{n}(\phi)=r g\left(\phi(0), \int_{-\infty}^{0} \phi(s) Q_{n}(s) d s\right)
$$

e $g^{\infty}: \mathcal{B} \rightarrow L^{2}(0,1)$ definida por

$$
g^{\infty}(\phi)=r g(\phi(0), \phi(-1))
$$

Então $g^{n}$ converge uniformemente para $g^{\infty}$ sobre conjuntos eqüicontínuos $e$ eqüilimitados de $\mathcal{B}$, quando $n \rightarrow \infty$. 


\section{Demonstração}

Sejam $M \subset \mathcal{B}$ conjunto eqüicontínuo e eqüilimitado e $\phi \in M$. E consideremos a diferença

$$
\begin{aligned}
& \left\|\int_{-\infty}^{0} \phi(s) Q_{n}(s) d s-\phi(-1)\right\|_{H^{1}} \\
& \quad \leq \int_{-\infty}^{0}\|\phi(s)-\phi(-1)\|_{H^{1}} Q_{n}(s) d s .
\end{aligned}
$$

Como $M$ é eqüicontínuo, dado $\varepsilon>0$ existe $\delta>0$, independente de $\phi$, tal que $\|\phi(s)-\phi(-1)\|_{H^{1}}<\frac{\varepsilon}{4}$, para todo $s \in[-1-\delta,-1+\delta]$.

Portanto,

$$
\int_{-1-\delta}^{-1+\delta}\|\phi(s)-\phi(-1)\|_{H^{1}} Q_{n}(s) d s<\frac{\varepsilon}{4}, \forall n \in \mathbb{N}
$$

E como $M$ é eqüilimitado, existe $K>0$ tal que $\|\phi\|_{\mathcal{B}}<K$ para todo $\phi \in M$. E considerando o $\varepsilon>0$ dado acima determinamos $n_{1}=n_{1}(\varepsilon, K) \in \mathbb{N}$ tal que $\int_{-1+\delta}^{0} Q_{n}(s) d s<\frac{\varepsilon}{8 K}, \forall n \geq n_{1}$.

Assim,

$$
\begin{aligned}
\int_{-1+\delta}^{0}\|\phi(s)-\phi(-1)\|_{H^{1}} Q_{n}(s) d s & \leq 2 K \int_{-1+\delta}^{0} Q_{n}(s) d s \\
& <\frac{\varepsilon}{4}, \forall n \geq n_{1}
\end{aligned}
$$

Observemos que dado $\varepsilon>0$ acima determinamos $n_{2}=n_{2}(\varepsilon, K) \in \mathbb{N}$ tal que $\int_{-\infty}^{-1-\delta} Q_{n}(s) d s<\frac{\varepsilon}{4 K}, \forall n \geq n_{2}$.

Logo,

$$
\int_{-\infty}^{-1-\delta}\|\phi(-1)\|_{H^{1}} Q_{n}(s) d s<\frac{\varepsilon}{4}, \forall n \geq n_{2} .
$$

Consideremos agora $h(s)=\frac{Q_{n}(s)}{Q_{1}(s)}=n^{n} \frac{(-s)^{n-1}}{(n-1) !} e^{(n-1) s}$. Temos que $h$ atinge o valor máximo em $s^{*}=-1$ e é crescente para $s<s^{*}$. 
Então, para $s \leq-1-\delta$

$$
\begin{aligned}
\frac{Q_{n}(s)}{Q_{1}(s)} & \leq \frac{Q_{n}(-1-\delta)}{Q_{1}(-1-\delta)} \\
& =n^{n} \frac{(1+\delta)^{n-1}}{(n-1) !} e^{(n-1)(-1-\delta)} \\
& =\frac{n^{n}(1+\delta)^{n-1}}{(n-1)^{(n-1)} \sqrt{2 \pi(n-1)}} e^{(n-1)} e^{(n-1)(-1-\delta)} e^{\frac{-\nu}{12(n-1)}} \\
& \leq\left(\frac{n}{n-1}\right)^{n} \sqrt{\frac{n-1}{2 \pi}}(1+\delta)^{n-1} e^{-\delta(n-1)} \\
& \leq 2 e \sqrt{\frac{n-1}{2 \pi}}(1+\delta)^{n-1} e^{-\delta(n-1)} \\
& =2 e \sqrt{\frac{n-1}{2 \pi}} e^{[-\delta+\ln (1+\delta)](n-1)} .
\end{aligned}
$$

Com $o \varepsilon>0$ dado acima determinamos $n_{3}=n_{3}(\varepsilon, K) \in \mathbb{N}$ tal que $\sup _{-\infty<s \leq-1-\delta} \frac{Q_{n}(s)}{Q_{1}(s)}<\frac{\varepsilon}{4 K}, \forall n \geq n_{3}$.

E portanto,

$$
\begin{aligned}
& \int_{-\infty}^{-1-\delta}\|\phi(s)\|_{H^{1}} Q_{n}(s) d s \\
& \quad \leq \sup _{-\infty<s \leq-1-\delta} \frac{Q_{n}(s)}{Q_{1}(s)} \int_{-\infty}^{-1-\delta}\|\phi(s)\|_{H^{1}} Q_{1}(s) d s \\
& \quad<\frac{\varepsilon}{4}, \forall n \geq n_{3} .
\end{aligned}
$$

Utilizando os resultados acima, para qualquer $\varepsilon>0$ podemos encon$\operatorname{trar} n_{0}=\max \left\{n_{1}, n_{2}, n_{3}\right\}$, independente de $\phi \in \mathcal{B}$, tal que

$$
\left\|\int_{-\infty}^{0} \phi(s) Q_{n}(s) d s-\phi(-1)\right\|_{H^{1}}<\varepsilon, \forall n \geq n_{0}
$$

Temos assim que $\left\|g^{n}(\phi)-g^{\infty}(\phi)\right\|_{H^{1}}<c \varepsilon$, para alguma constante $c$ pois $g$ é de classe $C^{1}$, com derivada limitada, e a proposição está demonstrada.

Observemos que as soluções do problema (2.1) são definidas para todo $t>0$ (página 49) e sabemos, do teorema 1.14, que para $t>r$ são de 
classe $C^{1}$; o mesmo valendo para as soluções do problema (2.5). Como o lema 3.11 acima só é válido em conjuntos eqüicontínuos e eqüilimitados e como queremos aproximar a dinâmica do problema contínuo e discretizado no atrator, ou seja, para um tempo grande, podemos supor, sem perda de generalidade, que $\varphi$ é Hölder contínua o que implica que as soluções sejam de classe $C^{1}$ para $t>0$.

O teorema 3.13 abaixo nos garante que as soluções do problema com retardo infinito (3.4) convergem às soluções do problema (2.1).

Convém observar que em sua demonstração vamos usar o teorema 1.5 e observemos também que $C\left([-1,0], H^{1}(0,1)\right)$ será denotado por $C_{1 / 2}$ e dado $\varepsilon>0, \mathcal{A}^{\varepsilon}$ irá denotar uma vizinhança de $\mathcal{A}$ de tamanho $\varepsilon$.

Necessitamos também do lema de Gronwall abaixo e para sua demonstração veja Henry [13].

Lema 3.12 Sejam $t<r, \phi:[t, r] \rightarrow \mathbb{R}^{+}$uma função contínua, $a>0, b>0$ $e 0<\beta \leq 1$. Se

$$
\phi(t) \leq a+b \int_{t}^{r}(s-t)^{\beta-1} \phi(s) d s, t \leq r
$$

então

$$
\phi(t) \leq a E_{\beta}\left((b \Gamma(\beta))^{1 / \beta}(r-t)\right)
$$

$\operatorname{com} E_{\beta}(z)=\sum_{k=0}^{\infty} \frac{z^{\beta k}}{\Gamma(k \beta+1)}$

Teorema 3.13 Se $\mathcal{A} \subset C\left([-1,0], H^{1}(0,1)\right)$ é o atrator global do problema com retardo unitário (3.1), dado $N \subset C\left([-1,0], H^{1}(0,1)\right)$ um conjunto limitado tal que $\mathcal{A}^{2} \subset N$ consideremos $v^{n}(x, t)=v^{n}(x, t, \hat{\varphi})$ solução do problema com retardo infinito (3.4), com condição inicial $v_{0}(x, t)=\hat{\varphi}(x, t)$, e 
$y(x, t)=y(x, t, \varphi)$ solução do problema com retardo unitário (3.1) e condição inicial $y(x, t)=\varphi(x, t r)$, para $t \in[-1,0]$ e $\varphi(\cdot, \cdot r) \in N$ Hölder contínua. Então $\left\|v^{n}(t)-y(t)\right\|_{H^{1}} \rightarrow 0$ uniformemente em $t$, em intervalos compactos, e em $\varphi(\cdot, \cdot r) \in N$ quando $n \rightarrow \infty$.

\section{Demonstração}

Dado $N \subset C\left([-1,0], H^{1}(0,1)\right)$, para todo $\varepsilon>0$ existe $t_{0}=t_{0}(\varepsilon, N)$ tal que $y_{t} \in \mathcal{A}^{\varepsilon / 2}$, para todo $t \geq t_{0}$ e pela dependência contínua em relação aos dados iniciais existe $\delta=\delta(\varepsilon, N)$ tal que

$$
\left\|y\left(x, t, \varphi_{1}\right)-y\left(x, t, \varphi_{2}\right)\right\|_{H^{1}}<\frac{\varepsilon}{2}
$$

para todo $t \in[0,1]$ e $\varphi_{1}, \varphi_{2} \in N$ com $\left\|\varphi_{1}-\varphi_{2}\right\|_{C_{1 / 2}}<\delta$.

Com isto basta mostrarmos que para todo $\varepsilon_{0}>0$ existe $n_{0}=n_{0}(\varepsilon, N)$ tal que $\left\|v^{n}(t)-y(t)\right\|_{H^{1}}<\varepsilon_{0}$, para todo $n \geq n_{0}, t \in\left[0, t_{0}\right]$ e $\varphi \in N$, com $\varepsilon_{0}<\min \left\{\delta, \frac{\varepsilon}{2}\right\}$, pois, utilizando o fato dos sistemas serem autônomos, usamos a dependência contínua para as condições iniciais transladadas e assim obtemos a convergência uniforme em $t$ e em $\varphi$.

Como $\varphi(\cdot, \cdot r) \in N$ é Hölder contínua existe $M=M(\varepsilon, N) \subset \mathcal{B}$, eqüicontínuo e eqüilimitado, tal que $v^{n}(t) \in M$ para todo $0 \leq t \leq t_{0}$ e $n \geq 0$. E consideremos $L_{g}$ a constante de Lipschitz da função $g, \bar{t}$ o menor inteiro maior ou igual à $t_{0}$ e $R^{*}>0$ tal que

$$
R_{0}=R^{*} \bar{t}\left(L_{g} r N_{1 / 2} \lambda^{-1 / 2} \Gamma(1 / 2) R_{1}\right)^{\bar{t}}>1
$$

com $R_{1}=\max _{0 \leq t \leq \bar{t}} E_{1 / 2}\left(\left(L_{g} r N_{1 / 2} \Gamma(1 / 2)\right)^{2} \bar{t}\right)$. E podemos supor, sem perda de generalidade, que $N_{1 / 2} \lambda^{-1 / 2} \Gamma(1 / 2)>1$ e $L_{g} r>1$.

Como $\left\|g^{n}\left(v_{t}^{n}\right)-g^{\infty}\left(v_{t}^{n}\right)\right\|_{H^{1}} \rightarrow 0$ uniformemente sobre conjuntos eqüicontínuos e eqüilimitados de $\mathcal{B}$, quando $n \rightarrow \infty$, existe $n_{0}=n_{0}(\varepsilon, N)$ tal 
que

$$
\left\|g^{n}\left(v_{t}^{n}\right)-g^{\infty}\left(v_{t}^{n}\right)\right\|_{L^{2}}<\frac{\varepsilon_{0}}{R_{0}}
$$

para todo $n \geq n_{0}$.

Então,

$$
\begin{aligned}
& \left\|v^{n}(t)-y(t)\right\|_{H^{1}} \\
& =\| e^{-(A+r I) t} v^{n}(0)+\int_{0}^{t} e^{-(A+r I)(t-s)} g^{n}\left(v_{s}^{n}\right) d s \\
& -e^{-(A+r I) t} y(0)-\int_{0}^{t} e^{-(A+r I)(t-s)} g^{\infty}\left(y_{s}\right) d s \|_{H^{1}} \\
& \leq N_{1 / 2} \int_{0}^{t} e^{-\lambda(t-s)}(t-s)^{-1 / 2}\left\|g^{n}\left(v_{s}^{n}\right)-g^{\infty}\left(v_{s}^{n}\right)\right\|_{L^{2}} d s \\
& +N_{1 / 2} \int_{0}^{t} e^{-\lambda(t-s)}(t-s)^{-1 / 2} \| r g\left(v^{n}(s), v^{n}(s-1)\right) \\
& \quad r g(y(s), y(s-1)) \|_{L^{2}} d s \\
& \quad \leq N_{1 / 2} \int_{0}^{t} e^{-\lambda(t-s)}(t-s)^{-1 / 2}\left\|g^{n}\left(v_{s}^{n}\right)-g^{\infty}\left(v_{s}^{n}\right)\right\|_{L^{2}} d s \\
& \quad+L_{g} r N_{1 / 2} \int_{0}^{t} e^{-\lambda(t-s)}(t-s)^{-1 / 2}\left\|v^{n}(s-1)-y(s-1)\right\|_{H^{1}} d s \\
& \quad+L_{g} r N_{1 / 2} \int_{0}^{t} e^{-\lambda(t-s)}(t-s)^{-1 / 2}\left\|v^{n}(s)-y(s)\right\|_{H^{1}} d s .
\end{aligned}
$$

Para concluir usamos indução, ou seja, se $0 \leq t \leq 1$ temos

$$
\begin{aligned}
& \left\|v^{n}(t)-y(t)\right\|_{H^{1}} \\
& \quad \leq N_{1 / 2} \lambda^{-1 / 2} \Gamma(1 / 2) \frac{\varepsilon_{0}}{R_{0}} \\
& \quad+L_{g} r N_{1 / 2} \int_{0}^{t} e^{-\lambda(t-s)}(t-s)^{-1 / 2}\left\|v^{n}(s)-y(s)\right\|_{H^{1}} d s
\end{aligned}
$$

Seja $z(t)=e^{\lambda t}\left\|v^{n}(t)-y(t)\right\|_{H^{1}}$, então

$$
\begin{aligned}
z(t) & \leq e^{\lambda t} N_{1 / 2} \lambda^{-1 / 2} \Gamma(1 / 2) \frac{\varepsilon_{0}}{R_{0}} \\
& +L_{g} r N_{1 / 2} \int_{0}^{t}(t-s)^{-1 / 2} z(s) d s
\end{aligned}
$$


assim, da desigualdade de Gronwall, segue que

$$
z(t) \leq e^{\lambda t} N_{1 / 2} \lambda^{-1 / 2} \Gamma(1 / 2) \frac{\varepsilon_{0}}{R_{0}} R_{1}
$$

E portanto,

$$
\left\|v^{n}(t)-y(t)\right\|_{H^{1}} \leq N_{1 / 2} \lambda^{-1 / 2} \Gamma(1 / 2) \frac{\varepsilon_{0}}{R_{0}} R_{1}<\varepsilon_{0}
$$

para todo $n \geq n_{0}$.

Suponhamos, agora, que para $\bar{t}-2 \leq t \leq \bar{t}-1$

$$
\begin{aligned}
& \left\|v^{n}(t)-y(t)\right\|_{H^{1}} \\
& \quad \leq N_{1 / 2} \lambda^{-1 / 2} \Gamma(1 / 2) \frac{\varepsilon_{0}}{R_{0}} R_{1} \\
& \quad+L_{g} r\left(N_{1 / 2} \lambda^{-1 / 2} \Gamma(1 / 2)\right)^{2} \frac{\varepsilon_{0}}{R_{0}} R_{1}^{2} \\
& \quad+\cdots \\
& \quad+L_{g}^{\bar{t}-2} r^{\bar{t}-2}\left(N_{1 / 2} \lambda^{-1 / 2} \Gamma(1 / 2)\right)^{\bar{t}-1} \frac{\varepsilon_{0}}{R_{0}} R_{1}^{\bar{t}-1} \\
& \leq \bar{t}\left(L_{g} r N_{1 / 2} \lambda^{-1 / 2} \Gamma(1 / 2)\right)^{\bar{t}-1} \frac{\varepsilon_{0}}{R_{0}} R_{1}^{\bar{t}-1} \\
& \quad<\varepsilon_{0},
\end{aligned}
$$

para todo $n \geq n_{0}$.

Assim, para $\bar{t}-1<t \leq \bar{t}$, temos

$$
\begin{aligned}
& \left\|v^{n}(t)-y(t)\right\|_{H^{1}} \\
& \quad \leq N_{1 / 2} \lambda^{-1 / 2} \Gamma(1 / 2) \frac{\varepsilon_{0}}{R_{0}} \\
& +L_{g} r\left(N_{1 / 2} \lambda^{-1 / 2} \Gamma(1 / 2)\right)^{2} \frac{\varepsilon_{0}}{R_{0}} R_{1} \\
& \quad+L_{g}^{2} r^{2}\left(N_{1 / 2} \lambda^{-1 / 2} \Gamma(1 / 2)\right)^{3} \frac{\varepsilon_{0}}{R_{0}} R_{1}^{2} \\
& \quad+\cdots \\
& \quad+L_{g}^{\bar{t}-1} r^{\bar{t}-1}\left(N_{1 / 2} \lambda^{-1 / 2} \Gamma(1 / 2)\right)^{\bar{t}} \frac{\varepsilon_{0}}{R_{0}} R_{1}^{\bar{t}-1} \\
& \quad+L_{g} r N_{1 / 2} \int_{0}^{t} e^{-\lambda(t-s)}(t-s)^{-1 / 2}\left\|v^{n}(s)-y(s)\right\|_{H^{1}} d s .
\end{aligned}
$$


Seja $z(t)=e^{\lambda t}\left\|v^{n}(t)-y(t)\right\|_{H^{1}}$, então

$$
\begin{aligned}
z(t) & \leq e^{\lambda t} N_{1 / 2} \lambda^{-1 / 2} \Gamma(1 / 2) \frac{\varepsilon_{0}}{R_{0}} \\
& +e^{\lambda t} L_{g} r\left(N_{1 / 2} \lambda^{-1 / 2} \Gamma(1 / 2)\right)^{2} \frac{\varepsilon_{0}}{R_{0}} R_{1} \\
& +\cdots \\
& +e^{\lambda t} L_{g}^{\bar{t}-1} r^{\bar{t}-1}\left(N_{1 / 2} \lambda^{-1 / 2} \Gamma(1 / 2)\right)^{\bar{t}} \frac{\varepsilon_{0}}{R_{0}} R_{1}^{\bar{t}-1} \\
& +L_{g} r N_{1 / 2} \int_{0}^{t}(t-s)^{-1 / 2} z(s) d s
\end{aligned}
$$

assim, da desigualdade de Gronwall, segue que

$$
\begin{aligned}
z(t) & \leq\left[e^{\lambda t} N_{1 / 2} \lambda^{-1 / 2} \Gamma(1 / 2) \frac{\varepsilon_{0}}{R_{0}}\right. \\
& +e^{\lambda t} L_{g} r\left(N_{1 / 2} \lambda^{-1 / 2} \Gamma(1 / 2)\right)^{2} \frac{\varepsilon_{0}}{R_{0}} R_{1} \\
& +\cdots \\
& \left.+e^{\lambda t} L_{g}^{\bar{t}-1} r^{\bar{t}-1}\left(N_{1 / 2} \lambda^{-1 / 2} \Gamma(1 / 2)\right)^{\bar{t}} \frac{\varepsilon_{0}}{R_{0}} R_{1}^{\bar{t}-1}\right] R_{1} .
\end{aligned}
$$

E portanto,

$$
\begin{aligned}
& \left\|v^{n}(t)-y(t)\right\|_{H^{1}} \\
& \quad \leq N_{1 / 2} \lambda^{-1 / 2} \Gamma(1 / 2) \frac{\varepsilon_{0}}{R_{0}} R_{1} \\
& \quad+L_{g} r\left(N_{1 / 2} \lambda^{-1 / 2} \Gamma(1 / 2)\right)^{2} \frac{\varepsilon_{0}}{R_{0}} R_{1}^{2} \\
& +\cdots \\
& \quad+L_{g}^{\bar{t}-1} r^{\bar{t}-1}\left(N_{1 / 2} \lambda^{-1 / 2} \Gamma(1 / 2)\right)^{\bar{t}} \frac{\varepsilon_{0}}{R_{0}} R_{1}^{\bar{t}} \\
& \leq \bar{t}\left(L_{g} r N_{1 / 2} \lambda^{-1 / 2} \Gamma(1 / 2)\right)^{\bar{t}} \frac{\varepsilon_{0}}{R_{0}} R_{1}^{\bar{t}} \\
& \quad<\varepsilon_{0},
\end{aligned}
$$

para todo $n \geq n_{0}$. 
A proposição abaixo nos garante a equivalência entre o problema com retardo infinito (3.4) e o problema discretizado (3.2). Para isto, observemos que se $v^{n}$ é solução do problema com retardo infinito então $W_{0}(x, t)=v^{n}(x, t)$ $\mathrm{e} W_{k}(x, t)=\int_{-\infty}^{t} v^{n}(x, s) r_{k}^{n}(s-t) d s, k=1, \ldots, n, \operatorname{com} r_{k}^{n}(s)=n^{k} \frac{(-s)^{k-1}}{(k-1) !} e^{n s}$, $s \leq 0$, são soluções do problema discretizado com condições iniciais

$$
\left\{\begin{array}{l}
W_{0}(x, 0)=\hat{\varphi}(x, 0) \\
W_{1}(x, 0)=\int_{-\infty}^{0} \hat{\varphi}(x, s) r_{1}^{n}(s) d s \\
\vdots \\
W_{n}(x, 0)=\int_{-\infty}^{0} \hat{\varphi}(x, s) Q_{n}(s) d s
\end{array}\right.
$$

Proposição 3.14 Sejam $v^{n}(x, t)$ solução do problema com retardo infinito com condição inicial $v_{0}(x, t)=\hat{\varphi}(x, t)$ e $\varphi(x,(-k r) / n), k=0, \ldots, n$ condições iniciais do problema discretizado. Então $\left\|\varphi((-k r) / n)-W_{k}(0)\right\|_{H^{1}} \rightarrow 0$ uniformemente quando $n \rightarrow \infty$, sendo $W_{k}(x, 0)=\int_{-\infty}^{0} v^{n}(x, s) r_{k}^{n}(s) d s$, $k=1, \ldots, n \operatorname{com} r_{k}^{n}(s)=n^{k} \frac{(-s)^{k-1}}{(k-1) !} e^{n s}, s \leq 0$ e $W_{0}(x, 0)=v^{n}(x, 0)$.

\section{Demonstração}

Temos que

$$
\begin{aligned}
\left\|\varphi((-k r) / n)-W_{k}(0)\right\|_{H^{1}} & \left\|\varphi \varphi((-k r) / n)-\int_{-\infty}^{0} \hat{\varphi}(s) r_{k}^{n}(s) d s\right\|_{H^{1}} \\
& \leq \int_{-\infty}^{0}\|\hat{\varphi}(-k / n)-\hat{\varphi}(s)\|_{H^{1}} r_{k}^{n}(s) d s \\
& =\int_{-\infty}^{(-k / n)-\delta}\|\hat{\varphi}(-k / n)-\hat{\varphi}(s)\|_{H^{1}} r_{k}^{n}(s) d s \\
& +\int_{(-k / n)-\delta}^{(-k / n)+\delta}\|\hat{\varphi}(-k / n)-\hat{\varphi}(s)\|_{H^{1}} r_{k}^{n}(s) d s \\
& +\int_{(-k / n)+\delta}^{0}\|\hat{\varphi}(-k / n)-\hat{\varphi}(s)\|_{H^{1}} r_{k}^{n}(s) d s .
\end{aligned}
$$

E o restante da demonstração desta proposição se assemelha à do lema 3.11, e neste caso consideramos $h_{k}^{n}(s)=r_{k}^{n}(s) e^{-s}$ e temos que $h_{k}^{n}$ atinge 
o valor máximo em $s^{*}=\frac{1-k}{n-1} \geq-1$ e é crescente para $s<s^{*}$, de onde segue que $r_{k}^{n}(-2)=e^{-2} h_{k}^{n}(-2) \leq n \sqrt{\frac{n}{2 \pi}}\left(\frac{2}{e}\right)^{n} \leq \frac{3}{2(n+1)}$.

A proximidade das soluções dos problemas com retardo finito e infinito nos permite concluir o teorema abaixo, que nos garante a semicontinuidade superior dos atratores do problema com retardo infinito.

Definição 3.15 Seja $\Lambda$ um espaço métrico, $X$ um espaço métrico completo $e J_{\lambda}, \lambda \in \Lambda$, uma familia de subconjuntos de $X$. Dizemos que $J_{\lambda}$ é semicontínuo superiormente em $\lambda_{0} \in \Lambda$ se $\operatorname{dist}\left(J_{\lambda}, J_{\lambda_{0}}\right) \rightarrow 0$ quando $\lambda \rightarrow \lambda_{0}$, sendo $\operatorname{dist}(A, B)=\sup _{y \in A} \inf _{x \in B} d(y, x), A, B$ subconjuntos de $X$.

Teorema 3.16 Para cada $n>0$, os atratores do problema com retardo infinto (3.4) são semi-contínuos superiormente em $n=\infty$.

\section{Demonstração}

Sejam $\mathcal{A}_{n} \subset \mathcal{B} \subset C\left([-1,0], H^{1}(0,1)\right)$ os atratores do problema com retardo infinito (3.4) e $\mathcal{A} \subset C\left([-1,0], H^{1}(0,1)\right)$ o atrator global do problema com retardo unitário (3.1).

Consideremos também $v^{n}(x, t)=v^{n}(x, t, \hat{\varphi})$ solução do problema com retardo infinito, e condição inicial $v_{0}(x, t)=\hat{\varphi}(x, t)$, e $y(x, t)=y(x, t, \varphi)$ solução do problema com retardo unitário e condição inicial $y(x, t)=\varphi(x, t r)$, para $t \in[-1,0]$.

Pelo teorema 3.13, temos que $\left\|v^{n}(t)-y(t)\right\|_{H^{1}} \rightarrow 0$ uniformemente em $t$, em intervalos compactos, quando $n \rightarrow \infty$. Logo, dado $\varepsilon>0$ existe 
$n_{0}>0$ tal que $\mathcal{A}_{n} \subset \mathcal{A}^{\varepsilon}$ para todo $n>n_{0}$ e assim $\sup _{v^{n} \in \mathcal{A}_{n}} \inf _{y \in \mathcal{A}} d\left(v^{n}, y\right)<\varepsilon$ para todo $n>n_{0}$, ou seja, $\operatorname{dist}\left(\mathcal{A}_{n}, \mathcal{A}\right) \rightarrow 0$ quando $n \rightarrow \infty$.

\subsection{Discretização espacial}

Nesta seção, baseada no trabalho de Bruschi, Carvalho e RuasFilho [2], consideramos uma discretização espacial do problema (3.2)

$$
\left\{\begin{array}{l}
\left(U_{0}(x, t)\right)_{t}=\operatorname{ar}\left(U_{0}(x, t)\right)_{x x}-r U_{0}(x, t)+r g\left(U_{0}(x, t), U_{n}(x, t)\right) \\
\left(U_{1}(x, t)\right)_{t}=n\left(U_{0}(x, t)-U_{1}(x, t)\right) \\
\vdots \\
\left(U_{n}(x, t)\right)_{t}=n\left(U_{n-1}(x, t)-U_{n}(x, t)\right)
\end{array}\right.
$$

para $0<x<1$ e $t>0$, com condições de fronteira

$$
\left(U_{0}\right)_{x}(0, t)=\left(U_{0}\right)_{x}(1, t)=0, t \geq 0
$$

e condições iniciais

$$
\left\{\begin{array}{l}
U_{0}(x, 0)=\varphi(x, 0) \\
U_{1}(x, 0)=\varphi\left(x, \frac{-r}{n}\right) \\
\vdots \\
U_{n}(x, 0)=\varphi(x,-r)
\end{array}\right.
$$

para $0<x<1$, e mostramos que o sistema de equações (3.2) e sua discretização possuem a mesma dinâmica assintótica.

Mais especificamente, aplicamos a discretização semi-implícita de Euler ao sistema (3.2), com $p$ passos igualmente espaçados, e para isto consideramos os pontos centrados $x_{j}=\frac{j-1 / 2}{p}, j=1, \ldots, p$ e denotamos $U_{i}^{j}(t)=U_{i}\left(x_{j}, t\right), i=0, \ldots, n$. 
Assim, temos que

$$
\begin{aligned}
\dot{U}_{0}^{1}(t)= & \operatorname{arp} p^{2}\left(U_{0}^{2}(t)-U_{0}^{1}(t)\right)-r U_{0}^{1}(t)+r g\left(U_{0}^{1}(t), U_{n}^{1}(t)\right) \\
\dot{U_{0}^{j}}(t)= & \operatorname{arp} p^{2}\left(U_{0}^{j+1}(t)-2 U_{0}^{j}(t)+U_{0}^{j-1}(t)\right)-r U_{0}^{j}(t)+r g\left(U_{0}^{j}(t), U_{n}^{j}(t)\right), \\
& j=2, \ldots, p-1, \\
\dot{U}_{0}^{p}(t)= & \operatorname{arp} p^{2}\left(U_{0}^{p-1}(t)-U_{0}^{p}(t)\right)-r U_{0}^{p}(t)+r g\left(U_{0}^{p}(t), U_{n}^{p}(t)\right), \\
\dot{U_{i}^{j}}(t)= & n\left(U_{i-1}^{j}(t)-U_{i}^{j}(t)\right), i=1, \ldots, n, j=1, \ldots, p .
\end{aligned}
$$

Observemos que as condições de fronteira, pela discretização, transformam-se em $U_{0}^{1}(t)=U_{0}^{0}(t)$ e $U_{0}^{p+1}(t)=U_{0}^{p}(t)$ e já foram incorporadas nas equações acima.

Denotando

$$
\begin{aligned}
U & =\left(U_{0}^{1}, \ldots, U_{0}^{p}, U_{1}^{1}, \ldots, U_{1}^{p}, \ldots, U_{n}^{1}, \ldots, U_{n}^{p}\right)^{T} \\
G(U) & =r\left(g\left(U_{0}^{1}, U_{n}^{1}\right), \ldots, g\left(U_{0}^{p}, U_{n}^{p}\right), 0, \ldots, 0\right)^{T}
\end{aligned}
$$

e $\Delta_{(n+1) p}$ a matriz $(n+1) p \times(n+1) p$ dada por

$$
\Delta_{(n+1) p}=\left(\begin{array}{c}
\Delta_{p \times(n+1) p}^{1}+\Delta_{p \times(n+1) p}^{2} \\
\Delta_{n p \times(n+1) p}^{3}
\end{array}\right)
$$

com

$$
\begin{gathered}
\Delta_{p \times(n+1) p}^{1}=\operatorname{arp} p^{2}\left(\begin{array}{cccccccccc}
1 & -1 & 0 & \cdots & 0 & 0 & 0 & 0 & \cdots & 0 \\
-1 & 2 & -1 & \cdots & 0 & 0 & 0 & 0 & \cdots & 0 \\
0 & -1 & 2 & \cdots & 0 & 0 & 0 & 0 & \cdots & 0 \\
& & & \ddots & & & & & & \\
0 & 0 & 0 & \cdots & 2 & -1 & 0 & 0 & \cdots & 0 \\
0 & 0 & 0 & \cdots & -1 & 2 & -1 & 0 & \cdots & 0 \\
0 & 0 & 0 & \cdots & 0 & -1 & 1 & 0 & \cdots & 0
\end{array}\right)_{p \times(n+1) p} \\
\Delta_{p \times(n+1) p}^{2}=r\left(\begin{array}{lllll}
I_{p} & O_{p} & \cdots & \left.O_{p}\right)_{p \times(n+1) p}
\end{array},\right.
\end{gathered}
$$

e

$$
\Delta_{n p \times(n+1) p}^{3}=n\left(\begin{array}{ccccc}
-I_{p} & I_{p} & O_{p} & \cdots & O_{p} \\
O_{p} & -I_{p} & I_{p} & \cdots & O_{p} \\
\vdots & & \ddots & \ddots & \\
O_{p} & \cdots & \cdots & -I_{p} & I_{p}
\end{array}\right)_{n p \times(n+1) p}
$$


sendo $I_{p}$ a matriz identidade de ordem $p$ e $O_{p}$ a matriz nula de ordem $p$, podemos escrever o sistema (3.5) na forma matricial

$$
\dot{U}=-\Delta_{(n+1) p} U+G(U)
$$

e temos assim a discretização espacial do problema (3.2) com condições iniciais

$$
\left\{\begin{array}{l}
U_{0}^{j}(0)=\varphi^{j}(0) \\
U_{1}^{j}(0)=\varphi^{j}\left(\frac{-1}{n}\right) \\
\vdots \\
U_{n}^{j}(0)=\varphi^{j}(-1)
\end{array}\right.
$$

sendo $\varphi^{j}(t)=\varphi\left(x_{j}, t r\right)$.

O que faremos agora é seguir de perto o trabalho de Bruschi, Carvalho e Ruas-Filho [2] e mostrar que as equações diferenciais ordinárias em (3.2) não interferem negativamente na convergência; nesse trabalho, ao invés dos problemas (3.6) e sua versão contínua (3.2), é estudado a convergência em relação ao problema discretizado

$$
\dot{V}=-\Delta_{p} V+F(V)
$$

e o problema contínuo

$$
v_{t}=a v_{x x}+f(v)
$$

Para nós, basta adaptarmos as convergências obtidas em Bruschi, Carvalho e Ruas-Filho [2] para o caso de sistemas.

Inicialmente vamos calcular os autovalores e autovetores da matriz $\Delta_{(n+1) p}$, assim obtemos uma base ortonormal de autovetores e escrevemos a equação discretizada (3.6) nestas novas coordenadas. Como conseqüência, temos um sistema fracamente acoplado para o qual existe uma variedade invariante exponencialmente atratora. 
Teorema 3.17 Os autovalores da matriz $\Delta_{(n+1) p}$ são dados por

$$
\lambda_{k}^{p}=4 a r p^{2} \sin ^{2} \frac{k \pi}{2 p}+r
$$

com $\lambda_{k}^{p} \neq n$, e os autovetores associados são

$$
\begin{aligned}
w_{k}^{(n+1), p}= & \left(\cos k \pi x_{1}, \ldots, \cos k \pi x_{p}, \frac{n \cos k \pi x_{1}}{n-\lambda_{k}^{p}}, \ldots, \frac{n \cos k \pi x_{p}}{n-\lambda_{k}^{p}}, \ldots\right. \\
& \left.\frac{n^{n} \cos k \pi x_{1}}{\left(n-\lambda_{k}^{p}\right)^{n}}, \cdots, \frac{n^{n} \cos k \pi x_{p}}{\left(n-\lambda_{k}^{p}\right)^{n}}\right)
\end{aligned}
$$

$k=0, \ldots,(n+1) p-1$. Além disso, $\lim _{p \rightarrow \infty} \lambda_{k}^{p}=\lambda_{k}=a r(k \pi)^{2}+r$, sendo $(k \pi)^{2} o(k+1)$-ésimo autovalor do operador $-\Delta$ com condições de fronteira de Neumann.

Observação 3.18 Temos $4 a p^{2} \sin ^{2} \frac{k \pi}{2 p}$ autovalor de $\Delta_{p}$ (equação (3.7)) associado ao autovetor normalizado

$$
\mu_{k}^{p}=\frac{1}{\left(\frac{1}{p} \sum_{j=1}^{p} \cos ^{2} k \pi x_{j}\right)^{1 / 2}}\left(\cos k \pi x_{1}, \ldots, \cos k \pi x_{p}\right)
$$

\section{Demonstração}

Consideremos, a princípio, apenas a equação

$$
\left(U_{0}(x, t)\right)_{t}=\operatorname{ar}\left(U_{0}(x, t)\right)_{x x}-r U_{0}(x, t)
$$

e sua discretização; e seja

$$
L=\operatorname{arp} p^{2}\left(\begin{array}{ccccccc}
1 & -1 & 0 & \cdots & 0 & 0 & 0 \\
-1 & 2 & -1 & \cdots & 0 & 0 & 0 \\
0 & -1 & 2 & \cdots & 0 & 0 & 0 \\
& & & \ddots & & & \\
0 & 0 & 0 & \cdots & 2 & -1 & 0 \\
0 & 0 & 0 & \cdots & -1 & 2 & -1 \\
0 & 0 & 0 & \cdots & 0 & -1 & 1
\end{array}\right)_{p \times p}+r I_{p}
$$


sendo $I_{p}$ a matriz identidade de ordem $p$.

Sabemos que as autofunções do operador $-\Delta$ com condições de fronteira de Neumann são dadas por

$$
\begin{aligned}
w_{l}:[0,1] & \rightarrow \mathbb{R} \\
x & \mapsto \cos l \pi x, l=0,1,2, \ldots
\end{aligned}
$$

Assim, considerando o ponto médio $x_{j}=\frac{j-1 / 2}{p}$ de cada intervalo $\left[\frac{j-1}{p}, \frac{j}{p}\right], j=1, \ldots, p$ e discretizando as $p$ primeiras autofunções $w_{l}(x)$ nos pontos $x_{j}$, obtemos

$$
w_{l}^{p}=\left(\cos l \pi x_{1}, \ldots, \cos l \pi x_{p}\right)
$$

Observemos agora que o vetor $L w_{l}^{p}$ tem as seguintes coordenadas

$$
\begin{aligned}
\left(L w_{l}^{p}\right)_{1} & =\operatorname{arp} p^{2}\left(\cos \frac{l \pi}{2 p}-\cos l \pi\left(\frac{1}{2 p}+\frac{1}{p}\right)\right)+r \cos l \pi x_{1} \\
\left(L w_{l}^{p}\right)_{j} & =\operatorname{arp} p^{2}\left(-\cos l \pi\left(x_{j}-\frac{1}{p}\right)+2 \cos l \pi x_{j}-\cos l \pi\left(x_{j}+\frac{1}{p}\right)\right) \\
& +r \cos l \pi x_{j}, j=2, \ldots, p-1 \\
\left(L w_{l}^{p}\right)_{p} & =a r p^{2}\left(-\cos l \pi\left(x_{p}-\frac{1}{p}\right)+\cos l \pi x_{p}\right)+r \cos l \pi x_{p} .
\end{aligned}
$$

E usando identidades trigonomêtricas segue que

$$
L w_{l}^{p}=\left(4 a r p^{2} \sin ^{2} \frac{l \pi}{2 p}+r\right) w_{l}^{p}, l=0, \ldots, p-1 .
$$

Assim, $L$ possui $p$ autovalores distintos dados por

$$
\lambda_{l}^{p}=4 a r p^{2} \sin ^{2} \frac{l \pi}{2 p}+r, l=0, \ldots, p-1 .
$$

Agora, considerando também o sistema de equações diferenciais ordinárias, podemos escrever

$$
\begin{aligned}
w_{k}^{(n+1), p}= & \left(\cos k \pi x_{1}, \ldots, \cos k \pi x_{p},(1,1)\right. \\
& w_{k}^{(n+1), p}, \ldots,(1, p) w_{k}^{(n+1), p}, \ldots \\
& \left.(n, 1) w_{k}^{(n+1), p}, \ldots,(n, p) w_{k}^{(n+1), p}\right)
\end{aligned}
$$


$\operatorname{com} j=1, \ldots, p$ e $k=0, \ldots,(n+1) p-1$.

E assim, considerando na definição de autovalor e autofunção o vetor $w_{k}^{(n+1), p}$ e o autovalor $\lambda_{k}^{p}$, obtemos

$$
{ }_{(i, j)} w_{k}^{(n+1), p}=\frac{n^{i} \cos k \pi x_{j}}{\left(n-\lambda_{k}^{p}\right)^{i}}, i=1, \ldots, n \text { e } j=1, \ldots, p .
$$

Para concluir, mostramos que $\lim _{p \rightarrow \infty} \lambda_{k}^{p}=\lambda_{k}=\operatorname{ar}(k \pi)^{2}+r$, sendo $(k \pi)^{2}$ o $(k+1)$-ésimo autovalor do operador $-\Delta$ com condições de fronteira de Neumann.

De fato, utilizando o limite fundamental, $\lim _{x \rightarrow 0} \frac{\sin x}{x}=1$, vem que

$$
\begin{aligned}
\lim _{p \rightarrow \infty} \lambda_{k}^{p} & =\lim _{p \rightarrow \infty}\left(4 a r p^{2} \sin ^{2} \frac{k \pi}{2 p}+r\right) \\
& =\lim _{u \rightarrow 0}\left(4 a r \frac{1}{u^{2}} \sin ^{2} \frac{k \pi}{2} u+r\right) \\
& =\lim _{u \rightarrow 0}\left(a r(k \pi)^{2} \frac{\sin ^{2}(k \pi u) / 2}{((k \pi u) / 2)^{2}}+r\right) \\
& =a r(k \pi)^{2}+r .
\end{aligned}
$$

Vamos agora associar a $\mathbb{R}^{(n+1) p}$ uma norma compatível com a norma adotada para o problema contínuo. Assim, definimos em $\mathbb{R}^{(n+1) p}$ o produto interno

$$
\langle x, y\rangle_{\mathbb{R}^{(n+1) p}}=\frac{1}{p} \sum_{i=1}^{(n+1) p} x_{i} y_{i}
$$

e normalizando $w_{k}^{(n+1), p}$ segundo este produto interno obtemos

$$
\begin{aligned}
& \nu_{k}^{(n+1), p} \\
& \quad=\frac{w_{k}^{(n+1), p}}{\left\|w_{k}^{(n+1), p}\right\|} \\
& \quad=\frac{1}{\left(\sum_{j=0}^{n} \frac{n^{2 j}}{\left(n-\lambda_{k}^{p}\right)^{2 j}}\right)^{1 / 2}}\left(\mu_{k}^{p}, \frac{n}{\left(n-\lambda_{k}^{p}\right)} \mu_{k}^{p}, \ldots, \frac{n^{n}}{\left(n-\lambda_{k}^{p}\right)^{n}} \mu_{k}^{p}\right),
\end{aligned}
$$


lembrando que $\mu_{k}^{p}=\frac{1}{\left(\frac{1}{p} \sum_{j=1}^{p} \cos ^{2} k \pi x_{j}\right)^{1 / 2}}\left(\cos k \pi x_{1}, \ldots, \cos k \pi x_{p}\right)$, como observado anteriormente.

Com o propósito de simplificarmos a notação escrevemos

$$
\begin{aligned}
& \nu_{k}^{(n+1), p}=\left({ }_{0} \nu_{k}^{(n+1), p}{ }_{11} \nu_{k}^{(n+1), p}, \ldots,{ }_{n} \nu_{k}^{(n+1), p}\right)
\end{aligned}
$$

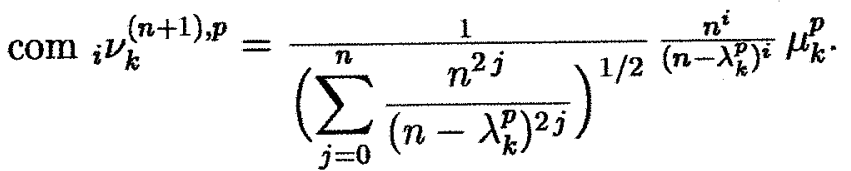

E no que segue, iremos denotar $\left(\sum_{j=0}^{n} \frac{n^{2 j}}{\left(n-\lambda_{k}^{p}\right)^{2 j}}\right)^{1 / 2}$ simplesmente por $N\left(\lambda_{k}^{p}\right)$

\section{Consideremos}

$$
\nu_{k}^{(n+1), p}(x)=\frac{1}{N\left(\lambda_{k}^{p}\right)}\left(\mu_{k}^{p}(x), \frac{n}{\left(n-\lambda_{k}^{p}\right)} \mu_{k}^{p}(x), \ldots, \frac{n^{n}}{\left(n-\lambda_{k}^{p}\right)^{n}} \mu_{k}^{p}(x)\right)
$$

sendo $\mu_{k}^{p}(x)=\frac{\sum_{j=1}^{p} \cos k \pi x_{j} \mathcal{X}_{I_{j}}(x)}{\left(\frac{1}{p} \sum_{j=1}^{p} \cos ^{2} k \pi x_{j}\right)^{1 / 2}}$, com $I_{j}$ o intervalo $\left[\frac{j-1}{p}, \frac{j}{p}\right]$ e $\mathcal{X}_{I_{j}}$ a função característica do intervalo $I_{j}$. De maneira análoga escremos

$$
{ }_{i} \nu_{k}^{(n+1), p}(x)=\frac{1}{N\left(\lambda_{k}^{p}\right)} \frac{n^{i}}{\left(n-\lambda_{k}^{p}\right)^{i}} \mu_{k}^{p}(x)
$$

$$
\operatorname{Temos} \nu_{k}^{(n+1), p}(x) \in \underbrace{L^{\infty}(0,1) \times \cdots \times L^{\infty}(0,1)}_{(n+1) \text { vezes }}, \text { pois } \mu_{k}^{p}(x) \in L^{\infty}(0,1) .
$$

Além disso, $\left\|\nu_{k}^{(n+1), p}(x)-\nu_{k}(x)\right\|_{\infty} \rightarrow 0$ quando $p \rightarrow \infty$ e $k$ e $n$ são fixos, sendo

$$
\nu_{k}(x)=\frac{1}{N\left(\lambda_{k}^{p}\right)}\left(\mu_{k}(x), \frac{n}{\left(n-\lambda_{k}^{p}\right)} \mu_{k}(x), \ldots, \frac{n^{n}}{\left(n-\lambda_{k}^{p}\right)^{n}} \mu_{k}(x)\right)
$$

$\operatorname{com} \mu_{k}(x)=\sqrt{2} \cos k \pi x$. E, como anteriormente, escrevemos

$$
{ }_{i} \nu_{k}(x)=\frac{1}{N\left(\lambda_{k}^{p}\right)} \frac{n^{i}}{\left(n-\lambda_{k}^{p}\right)^{i}} \mu_{k}(x) \text {. }
$$


É suficiente mostrarmos que $\left|\mu_{k}^{p}(x)-\mu_{k}(x)\right| \rightarrow 0$ quando $p \rightarrow \infty \mathrm{e}$ $k$ é fixo e isto segue diretamente dos resultados $\lim _{p \rightarrow \infty} \frac{1}{p} \sum_{j=1}^{p} \cos ^{2} k \pi x_{j}=\frac{1}{2} \mathrm{e}$ $\left|\cos k \pi x-\cos k \pi x_{j}\right| \leq \frac{k \pi}{p}$ se $x \in\left[\frac{j-1}{p}, \frac{j}{p}\right]$ e $j=1, \ldots, p$.

Assim,

$$
\begin{aligned}
\left\|\nu_{k}^{(n+1), p}(x)-\nu_{k}(x)\right\|_{\infty} & =\sup _{x \in[0,1]}\left|\nu_{k}^{(n+1), p}(x)-\nu_{k}(x)\right| \\
& =\sup _{x \in[0,1]} \sum_{i=0}^{n}\left|{ }_{i} \nu_{k}^{(n+1), p}(x)-{ }_{i} \nu_{k}(x)\right| \\
& <\varepsilon
\end{aligned}
$$

para $p$ suficientemente grande e $k$ e $n$ fixos.

Vamos agora considerar a base de autovetores $\nu_{k}^{(n+1), p}, \mathrm{em} \mathbb{R}^{(n+1) p}$, com $0 \leq k \leq(n+1) p-1$, e observemos que ela é ortonormal com respeito ao produto interno definido em $\mathbb{R}^{(n+1) p}$. A seguir, escrevemos a equação discretizada (3.6)

$$
\dot{U}=-\Delta_{(n+1) p} U+G(U),
$$

nestas novas coordenadas, ou seja, considerando

$$
v_{1}=\left\langle U, \nu_{0}^{(n+1), p}\right\rangle_{\mathbb{R}^{(n+1) p}, \ldots, v_{(n+1) p}}=\left\langle U, \nu_{(n+1) p-1}^{(n+1), p}\right\rangle_{\mathbb{R}^{(n+1) p}}
$$

e

$$
v=\left(v_{1}, \ldots, v_{(n+1) p}\right)^{T}
$$

obtemos a equação

$$
\dot{v}=-\tilde{\Delta}_{(n+1) p} v+\tilde{G}(v)
$$

sendo $\tilde{\Delta}_{(n+1) p}$ a matriz diagonal $(n+1) p \times(n+1) p$ dada por

$$
\tilde{\Delta}_{(n+1) p}=\operatorname{diag}\left(\lambda_{0}^{p}, \ldots, \lambda_{(n+1) p-1}^{p}\right)
$$

$\mathrm{e}$

$$
\tilde{G}(v)=\left(\tilde{G}_{1}(v), \ldots, \tilde{G}_{(n+1) p}(v)\right)^{T}
$$


com

$$
\begin{aligned}
\tilde{G}_{i}(v)= & \left\langle G(U), \nu_{i-1}^{(n+1), p}\right\rangle_{\mathbb{R}^{(n+1) p}} \\
= & \frac{1}{p} \sum_{j=1}^{(n+1) p}[G(U)]_{j}\left[\nu_{i-1}^{(n+1), p}\right]_{j} \\
= & \frac{r}{p} \sum_{j=1}^{p} g\left(\sum_{k=1}^{(n+1) p} \frac{v_{k}\left[\mu_{k-1}^{p}\right]_{j}}{N\left(\lambda_{k-1}^{p}\right)}, \sum_{k=1}^{(n+1) p} \frac{v_{k} \frac{n^{n}}{\left(n-\lambda_{k-1}^{p}\right)^{n}}\left[\mu_{k-1}^{p}\right]_{j}}{N\left(\lambda_{k-1}^{p}\right)}\right) \\
& \frac{\left[\mu_{i-1}^{p}\right]_{j}}{N\left(\lambda_{i-1}^{p}\right)},
\end{aligned}
$$

sendo $\left[\mu_{k-1}^{p}\right]_{j}$ a $j$-ésima coordenada de $\mu_{k-1}^{p}$.

Agora, vamos considerar uma discretização com $p=m^{3}$ pontos e a decomposição de $\mathbb{R}^{(n+1) m^{3}}=\mathbb{R}^{(n+1) m} \oplus \mathbb{R}^{(n+1)\left(m^{3}-m\right)}$ com

$$
\mathbb{R}^{(n+1) m}=\operatorname{span}\left[\nu_{0}^{(n+1), m^{3}}, \ldots, \nu_{(n+1) m-1}^{(n+1), m^{3}}\right]
$$

e

$$
\mathbb{R}^{(n+1)\left(m^{3}-m\right)}=\operatorname{span}\left[\nu_{(n+1) m}^{(n+1), m^{3}}, \ldots, \nu_{(n+1) m^{3}-1}^{(n+1), m^{3}}\right]
$$

Com esta decomposição obtemos o sistema fracamente acoplado

$$
\left\{\begin{array}{l}
\dot{v}_{(n+1) m}+\tilde{B}_{(n+1) m} v_{(n+1) m}=\tilde{h}_{(n+1) m}\left(v_{(n+1) m}, w_{(n+1) m}\right) \\
\dot{w}_{(n+1) m}+\tilde{A}_{(n+1) m} w_{(n+1) m}=\tilde{f}_{(n+1) m}\left(v_{(n+1) m}, w_{(n+1) m}\right)
\end{array}\right.
$$

sendo $\tilde{B}_{(n+1) m}$ a matriz diagonal $(n+1) m \times(n+1) m$ dada por

$$
\tilde{B}_{(n+1) m}=\operatorname{diag}\left(\lambda_{0}^{m^{3}}, \ldots, \lambda_{(n+1) m-1}^{m^{3}}\right)
$$

$\tilde{A}_{(n+1) m}$ a matriz diagonal $(n+1)\left(m^{3}-m\right) \times(n+1)\left(m^{3}-m\right)$ dada por

$$
\tilde{A}_{(n+1) m}=\operatorname{diag}\left(\lambda_{(n+1) m}^{m^{3}}, \ldots, \lambda_{(n+1) m^{3}-1}^{m^{3}}\right)
$$

e as funções $\tilde{h}_{(n+1) m}$ e $\tilde{f}_{(n+1) m}$ dadas por

$$
\tilde{h}_{(n+1) m}=\left(\tilde{G}_{1}\left(v_{(n+1) m}, w_{(n+1) m}\right), \ldots, \tilde{G}_{(n+1) m}\left(v_{(n+1) m}, w_{(n+1) m}\right)\right)^{T}
$$




$$
\tilde{f}_{(n+1) m}=\left(\tilde{G}_{(n+1) m+1}\left(v_{(n+1) m}, w_{(n+1) m}\right), \ldots, \tilde{G}_{(n+1) m^{3}}\left(v_{(n+1) m}, w_{(n+1) m}\right)\right)^{T}
$$

Para mostrarmos existência de variedade invariante exponencialmente atratora para o sistema (3.9) necessitamos do teorema abaixo e para sua demonstração veja Bruschi, Carvalho e Ruas-Filho [2].

Definição 3.19 Sejam $X$ e $Y$ espaços de Banach, $A: D(A) \subset X \rightarrow X$ um operador setorial, $B: D(B) \subset Y \rightarrow Y$ gerador de um $C^{0}$-grupo de operadores lineares limitados e $f: X^{\alpha} \times Y^{\alpha} \rightarrow X$ e $g: X^{\alpha} \times Y^{\alpha} \rightarrow Y$ funções localmente Lipschitz contínuas. Um conjunto $S \subset X^{\alpha} \times Y^{\alpha}$ é variedade invariante para uma equação diferencial

$$
\begin{aligned}
& \dot{x}=A x+f(x, y) \\
& \dot{y}=B y+g(x, y)
\end{aligned}
$$

se existe $\sigma: Y^{\alpha} \rightarrow X^{\alpha}$ tal que $S=\left\{(x, y) \in X^{\alpha} \times Y^{\alpha} ; x=\sigma(y)\right\}$ e, para qualquer $\left(x_{0}, y_{0}\right) \in S$, existe uma solução $(x(\cdot), y(\cdot))$ da equação diferencial sobre $\mathbb{R}$ tal que $(x(t), y(t)) \in S, \forall t \in \mathbb{R}$. Uma variedade invariante $S$ é exponencialmente atratora se existem constantes positivas $\gamma$ e $K$ tal que

$$
\|x(t)-\sigma(y(t))\|_{X^{\alpha}} \leq K e^{-\gamma t}\|x(0)-\sigma(y(0))\|_{X^{\alpha}}
$$

para $(x(t), y(t))$ solução da equação diferencial.

Teorema 3.20 Sejam $X_{m}, Y_{m}$ uma seqüência de espaços de Banach e sejam $A_{m}: D\left(A_{m}\right) \subset X_{m} \rightarrow X_{m}$ uma seqüência de operadores setoriais $e$ 
$B_{m}: D\left(B_{m}\right) \subset Y_{m} \rightarrow Y_{m}$ uma seqüência de geradores de $C^{0}$-grupos de operadores lineares limitados. E sejam $f_{m}: X_{m}^{\alpha} \times Y_{m}^{\alpha} \rightarrow X_{m}$ e $g_{m}: X_{m}^{\alpha} \times Y_{m}^{\alpha} \rightarrow Y_{m}$ uma seqüência de funções satisfazendo

$$
\left\|f_{m}(x, y)-f_{m}(z, w)\right\|_{X_{m}} \leq L_{f}\left(\|x-z\|_{X_{m}^{\alpha}}+\|y-w\|_{Y_{m}^{\alpha}}\right)
$$

$e$

$$
\left\|f_{m}(x, y)\right\|_{X_{m}} \leq N_{f}
$$

para todo $(x, y),(z, w) \in X_{m}^{\alpha} \times Y_{m}^{\alpha} e$

$$
\left\|g_{m}(x, y)-g_{m}(z, w)\right\|_{Y_{m}} \leq L_{g}\left(\|x-z\|_{X_{m}^{\alpha}}+\|y-w\|_{Y_{m}^{\alpha}}\right)
$$

$e$

$$
\left\|g_{m}(x, y)\right\|_{Y_{m}} \leq N_{g}
$$

para todo $(x, y),(z, w) \in X_{m}^{\alpha} \times Y_{m}^{\alpha}$. Assuma que

$$
\begin{aligned}
\left\|e^{-A_{m} t} w\right\|_{X_{m}^{\alpha}} & \leq M_{a} e^{-\beta(m) t}\|w\|_{X_{m}^{\alpha}}, t \geq 0 \\
\left\|e^{-A_{m} t} w\right\|_{X_{m}^{\alpha}} & \leq M_{a} t^{-\alpha} e^{-\beta(m) t}\|w\|_{X_{m}}, t>0 \\
\left\|e^{-B_{m} t} z\right\|_{Y_{m}^{\alpha}} & =\left\|e^{B_{m}(-t)} z\right\|_{Y_{m}^{\alpha}} \leq M_{b} e^{-\rho(m) t}\|z\|_{Y_{m}^{\alpha}}, t \leq 0 \\
\left\|e^{-B_{m} t} z\right\|_{Y_{m}^{\alpha}} & \leq M_{b}(-t)^{-\alpha} e^{-\rho(m) t}\|z\|_{Y_{m}}, t<0
\end{aligned}
$$

para qualquer $w \in X_{m}^{\alpha}$ e $z \in Y_{m}^{\alpha}, \operatorname{com} \beta(m)-\rho(m) \rightarrow \infty$ quando $m \rightarrow \infty$.

Considere o sistema fracamente acoplado

$$
\left\{\begin{array}{l}
\dot{x}=-A_{m} x+f_{m}(x, y) \\
\dot{y}=-B_{m} y+g_{m}(x, y)
\end{array}\right.
$$

Então, para $m$ suficientemente grande, existe uma variedade invariante exponencialmente atratora para (3.10),

$$
S=\left\{(x, y) ; x=\sigma_{m}(y), y \in Y_{m}^{\alpha}\right\}
$$


$\operatorname{com} \sigma_{m}: Y_{m}^{\alpha} \rightarrow X_{m}^{\alpha}$ satisfazendo

$$
s(m)=\sup _{y \in Y_{m}^{\alpha}}\left\|\sigma_{m}(y)\right\|_{X_{m}^{\alpha}}
$$

$e$

$$
\left\|\sigma_{m}(y)-\sigma_{m}(z)\right\|_{X_{m}^{\alpha}} \leq l(m)\|y-z\|_{Y_{m}^{\alpha}}
$$

com $s(m), l(m) \rightarrow 0$ quando $m \rightarrow \infty$. Se $f$ e g são suaves, então $\sigma_{m}$ é suave e sua derivada $D \sigma_{m}$ satisfaz

$$
\sup _{y \in Y_{m}}\left\|D \sigma_{m}(y)\right\|_{L\left(Y_{m}^{\alpha}, X_{m}^{\alpha}\right)} \leq l(m)
$$

O teorema 3.21 abaixo nos garante que existe uma variedade invariante, $(n+1) m$-dimensional exponencialmente atratora, para o sistema fracamente acoplado (3.9). Observemos que separamos o espaço de fase do problema discretizado (3.6) em um espaço gerado pelas $(n+1) m$ autofunções do problema discretizado e seu complemento ortogonal e a seguir projetamos o problema discretizado (3.6) sobre estes espaços e isto nos fornece o par de equações que aparece na afirmação do teorema 3.20 .

Teorema 3.21 Seja $g$ de classe $C^{2}$, limitada com primeira e segunda derivadas limitadas. Então o problema (3.9), para $m$ suficientemente grande, possui uma variedade invariante

$$
M_{(n+1) m}=\left\{\left(v_{(n+1) m}, w_{(n+1) m}\right) \in \mathbb{R}^{(n+1) m^{3}} ; w_{(n+1) m}=\tilde{\sigma}_{(n+1) m}\left(v_{(n+1) m}\right)\right\}
$$

a qual é exponencialmente atratora, sendo $\tilde{\sigma}_{(n+1) m}: \mathbb{R}^{(n+1) m} \rightarrow \mathbb{R}^{(n+1)\left(m^{3}-m\right)}$ uma função suave e o fluxo sobre $M_{(n+1) m}$ dado por

$$
u(t)=v_{(n+1) m}(t)+\tilde{\sigma}_{(n+1) m}\left(v_{(n+1) m}(t)\right)
$$


sendo $v_{(n+1) m}(t)$ solução de

$$
\dot{v}_{(n+1) m}+\tilde{B}_{(n+1) m} v_{(n+1) m}=\tilde{h}_{(n+1) m}\left(v_{(n+1) m}, \tilde{\sigma}_{(n+1) m}\left(v_{(n+1) m}\right)\right) .
$$

Além disso, a constante de Lipschitz, $l((n+1) m)$, de $\tilde{\sigma}_{(n+1) m} e$ $s(n)=\left\|\tilde{\sigma}_{(n+1) m}\right\|=\sup _{v_{(n+1) m} \in \mathbb{R}^{(n+1) m}}\left\|\tilde{\sigma}_{(n+1) m}\left(v_{(n+1) m}\right)\right\|$ convergem para zero quando $m \rightarrow \infty$.

\section{Demonstração}

Considerando $\alpha=0$ no teorema 3.20 podemos escrever $Y_{m}=\mathbb{R}^{(n+1) m}$, $X_{m}=\mathbb{R}^{(n+1)\left(m^{3}-m\right)}, \tilde{h}_{(n+1) m}: Y_{m} \times X_{m} \rightarrow Y_{m}$ e $\tilde{f}_{(n+1) m}: Y_{m} \times X_{m} \rightarrow X_{m}$.

Notemos que vamos fazer a seguinte distinção com relação às normas utilizadas, $\|x\|_{Y_{m}}=\left(\sum_{i=1}^{(n+1) m} x_{i}^{2}\right)^{1 / 2}$, o mesmo valendo para o espaço $X_{m}$, neste caso estamos usando a base de autovetores, e $\|x\|_{\mathbb{R}^{k}}=\left(\frac{1}{p} \sum_{i=1}^{k} x_{i}^{2}\right)^{1 / 2}$, e estamos usando a base canônica. $\mathrm{E}$ vamos considerar $Z$ a matriz mudança de base, com $z_{k j}=\left[\nu_{j-1}\right]_{k}$ a $k$-ésima coordenada de $\nu_{j-1}$.

Como conseqüência,

$$
\begin{aligned}
& \left\|\tilde{h}_{(n+1) m}\left(v_{(n+1) m}, w_{(n+1) m}\right)\right\|_{Y_{m}} \\
& \quad=\left(\sum_{i=1}^{(n+1) m}\left(\tilde{G}_{i}\left(v_{(n+1) m}, w_{(n+1) m}\right)\right)^{2}\right)^{1 / 2} \\
& \quad \leq\left(\sum _ { i = 1 } ^ { ( n + 1 ) m ^ { 3 } } \left(\left\langle G(U), \nu_{i-1}^{(n+1), m^{3}}\right\rangle_{\left.\left.\mathbb{R}^{(n+1) m^{3}}\right)^{2}\right)^{1 / 2}}\right.\right. \\
& \leq\left\|G\left(Z\left(v_{(n+1) m}, w_{(n+1) m}\right)^{T}\right)\right\|_{\mathbb{R}^{(n+1) m^{3}}} \\
& \quad=\left(\frac{1}{m^{3}} \sum_{i=1}^{(n+1) m^{3}}\left(\left[G\left(Z\left(v_{(n+1) m}, w_{(n+1) m}\right)^{T}\right)\right]_{i}\right)^{2}\right)^{1 / 2} \\
& \leq\left(\frac{1}{m^{3}} \sum_{i=1}^{m^{3}}\|g\|_{\infty}^{2}\right)^{1 / 2} \\
& \quad=\|g\|_{\infty} .
\end{aligned}
$$


Analogamente,

$$
\left\|\tilde{f}_{(n+1) m}\left(v_{(n+1) m}, w_{(n+1) m}\right)\right\|_{X_{m}} \leq\|g\|_{\infty}
$$

Vamos agora mostrar que $\tilde{h}_{(n+1) m}$ e $\tilde{f}_{(n+1) m}$ são localmente Lipschitz contínuas. Temos que

$$
\begin{aligned}
& \left\|\tilde{h}_{(n+1) m}\left(v_{(n+1) m}, w_{(n+1) m}\right)-\tilde{h}_{(n+1) m}\left(z_{(n+1) m}, u_{(n+1) m}\right)\right\|_{Y_{m}} \\
& \quad=\left(\sum_{i=1}^{(n+1) m}\left(\tilde{G}_{i}\left(v_{(n+1) m}, w_{(n+1) m}\right)-\tilde{G}_{i}\left(z_{(n+1) m}, u_{(n+1) m}\right)\right)^{2}\right)^{1 / 2} \\
& \leq\left\|G\left(Z\left(v_{(n+1) m}, w_{(n+1) m}\right)^{T}\right)-G\left(Z\left(z_{(n+1) m}, u_{(n+1) m}\right)^{T}\right)\right\|_{\mathbb{R}^{(n+1) m^{3}}} \\
& =\left(\frac { 1 } { m ^ { 3 } } \sum _ { i = 1 } ^ { ( n + 1 ) m ^ { 3 } } \left(\left[G\left(Z\left(v_{(n+1) m}, w_{(n+1) m}\right)^{T}\right)\right]_{i}\right.\right. \\
& \left.\left.-\left[G\left(Z\left(z_{(n+1) m}, u_{(n+1) m}\right)^{T}\right)\right]_{i}\right)^{2}\right)^{1 / 2} \\
& \left.\quad \leq\left(\frac{1}{m^{3}} \sum_{i=1}^{(n+1) m^{3}} L_{g}^{2}\left(\left[Z\left(v_{(n+1) m}, w_{(n+1) m}\right)^{T}\right]_{i}\right)-\left[Z\left(z_{(n+1) m}, u_{(n+1) m}\right)^{T}\right]_{i}\right)^{2}\right)^{1 / 2} \\
& \quad=\left(\frac{1}{m^{3}} \sum_{i=1}^{(n+1) m^{3}} L_{g}^{2}\left(\left[Z\left(v_{(n+1) m}-z_{(n+1) m}, w_{(n+1) m}-u_{(n+1) m}\right)^{T}\right]_{i}\right)^{2}\right)^{1 / 2} \\
& \quad=L_{g}\left\|Z\left(v_{(n+1) m}-z_{(n+1) m}, w_{(n+1) m}-u_{(n+1) m}\right)^{T}\right\|_{\mathbb{R}^{(n+1) m}} \\
& \leq \sqrt{2} L_{g}\left(\left\|v_{(n+1) m}-z_{(n+1) m}\right\|_{Y_{m}}+\left\|w_{(n+1) m}-u_{(n+1) m}\right\|_{X_{m}}\right)
\end{aligned}
$$

sendo $L_{g}$ a constante de Lipschitz da função $g$.

De modo análogo obtemos a estimativa para a constante de Lipschitz de $\tilde{f}_{(n+1) m}$.

Para concluir, observemos que as constantes dadas no teorema 3.20 são, neste caso, $\beta((n+1) m)=\lambda_{(n+1) m}^{m^{3}}$ e $\rho((n+1) m)=\lambda_{(n+1) m-1}^{m^{3}}$. Assim, $\beta((n+1) m) \sim a r[(n+1) m \pi]^{2}+r$ e $\rho((n+1) m) \sim a r[(n+1) m-1]^{2} \pi^{2}+r$, quando $m \rightarrow \infty$, e portanto $\beta((n+1) m)-\rho((n+1) m) \rightarrow \infty$ quando $m \rightarrow \infty$.

Portanto, do teorema 3.20 , segue que para $m$ suficientemente grande 
existe uma variedade invariante

$$
M_{(n+1) m}=\left\{\left(v_{(n+1) m}, w_{(n+1) m}\right) \in \mathbb{R}^{(n+1) m^{3}} ; w_{(n+1) m}=\tilde{\sigma}_{(n+1) m}\left(v_{(n+1) m}\right)\right\}
$$

a qual é exponencialmente atratora, sendo $\tilde{\sigma}_{(n+1) m}: \mathbb{R}^{(n+1) m} \rightarrow \mathbb{R}^{(n+1)\left(m^{3}-m\right)}$ o fluxo sobre $M_{(n+1) m}$ dado por $u(t)=v_{(n+1) m}(t)+\tilde{\sigma}_{(n+1) m}\left(v_{(n+1) m}(t)\right)$ com $v_{(n+1) m}(t)$ solução de

$$
\dot{v}_{(n+1) m}+\tilde{B}_{(n+1) m} v_{(n+1) m}=\tilde{h}_{(n+1) m}\left(v_{(n+1) m}, \tilde{\sigma}_{(n+1) m}\left(v_{(n+1) m}\right)\right) .
$$

Em analogia com o problema discretizado obtemos, para o problema contínuo, um sistema fracamente acoplado para o qual existe uma variedade invariante exponencialmente atratora.

A seguir, projetamos os problemas discretizado e contínuo sobre a variedade invariante e mostramos a convergência uniforme dos autovalores e autovetores da matriz $\Delta_{(n+1) p}$ aos autovalores e autofunções do Laplaciano transladado, com condições de Neumann, mas para isto é preciso que a dimensão do problema discretizado exceda a dimensão da variedade sobre a qual o problema contínuo está sendo considerado. É importante observarmos que ao considerarmos a matriz $\Delta_{(n+1) p} \operatorname{com} p=m^{3}$ passos, os primeiros $(n+1) m$ autovalores e autovetores da matriz $\Delta_{(n+1) m^{3}}$ convergem uniformemente para os primeiros $(n+1) m$ autovalores e autofunções do Laplaciano transladado, com condições de fronteira de Neumann, quando $m \rightarrow \infty$, mas não temos a convergência uniforme dos $(n+1) m^{3}$ autovalores e autovetores.

Vamos considerar o problema (3.2)

$$
\left\{\begin{array}{l}
\left(U_{0}(x, t)\right)_{t}=a r\left(U_{0}(x, t)\right)_{x x}-r U_{0}(x, t)+r g\left(U_{0}(x, t), U_{n}(x, t)\right) \\
\left(U_{1}(x, t)\right)_{t}=n\left(U_{0}(x, t)-U_{1}(x, t)\right) \\
\vdots \\
\left(U_{n}(x, t)\right)_{t}=n\left(U_{n-1}(x, t)-U_{n}(x, t)\right)
\end{array}\right.
$$


no espaço $X=\underbrace{L^{2}(0,1) \times \cdots \times L^{2}(0,1)}_{(n+1) \text { vezes }}$ e sejam as autofunções normalizadas

$$
\nu_{k}(x)=\frac{1}{N\left(\lambda_{k}^{p}\right)}\left(\mu_{k}(x), \frac{n}{\left(n-\lambda_{k}^{p}\right)} \mu_{k}(x), \ldots, \frac{n^{n}}{\left(n-\lambda_{k}^{p}\right)^{n}} \mu_{k}(x)\right)
$$

e os autovalores $\lambda_{k}=a r(k \pi)^{2}+r$, lembrando que $(k \pi)^{2}$ é autovalor do operador $-\Delta$ em $[0,1]$ associado ao autovetor $\mu_{k}(x)=\sqrt{2} \cos k \pi x$, obtidos em (3.8).

Vamos agora considerar a seguinte decomposição de $X=W \oplus W^{\perp}$, com

$$
\begin{aligned}
W & =\operatorname{span}\left[\nu_{0}, \ldots, \nu_{(n+1) m-1}\right] \\
W^{\perp} & =\left\{\nu \in X ;\langle\nu, w\rangle_{X}=0, \forall w \in W\right\}
\end{aligned}
$$

e o produto interno definido em $X$ como sendo $\langle x, y\rangle_{X}=\sum_{i=0}^{n+1}\left\langle x_{i}, y_{i}\right\rangle_{L^{2}(0,1)}$. Então, se $U \in X$ temos

$$
U=v_{1} \nu_{0}+\cdots+v_{(n+1) m} \nu_{(n+1) m-1}+w,
$$

sendo $v_{i}=\left\langle U, \nu_{i-1}\right\rangle_{X}, i=1, \ldots,(n+1) m$ e $w=U-\sum_{i=1}^{(n+1) m} v_{i} \nu_{i-1}$.

Seja $U$ uma solução de (3.2), então para cada $t$ podemos escrever

$$
U(t, x)=v_{1}(t) \nu_{0}(x)+\cdots+v_{(n+1) m}(t) \nu_{(n+1) m-1}(x)+w(t, x)
$$

e

$$
\begin{aligned}
\dot{v}_{i} & =-\lambda_{i} v_{i}+\left\langle G(U), \nu_{i-1}\right\rangle_{X} \\
w_{t} & =-A_{(n+1) m} w+G(U)-\sum_{i=1}^{(n+1) m}\left\langle G(U), \nu_{i-1}\right\rangle_{X} \nu_{i-1}
\end{aligned}
$$

$\operatorname{com} A_{(n+1) m}=(A+r I)_{\left.\right|_{D(A) \cap W^{\perp}}}$.

Escrevendo $v=\left(v_{1}, \ldots, v_{(n+1) m}\right), U=(v, w)$ e $B_{(n+1) m}$ a matriz diagonal $(n+1) m \times(n+1) m$ dada por $B_{(n+1) m}=\operatorname{diag}\left(\lambda_{0}, \ldots, \lambda_{(n+1) m-1}\right)$, 
obtemos o sistema

$$
\left\{\begin{array}{l}
\dot{v}+B_{(n+1) m} v=h_{(n+1) m}(v, w) \\
w_{t}+A_{(n+1) m} w=f_{(n+1) m}(v, w)
\end{array}\right.
$$

$\operatorname{com} h_{(n+1) m}$ e $f_{(n+1) m}$ dadas por

$$
h_{(n+1) m}(v, w)=r\left(\left\langle g(v, w), \nu_{0}\right\rangle_{X}, \ldots,\left\langle g(v, w), \nu_{(n+1) m-1}\right\rangle_{X}\right)^{T}
$$

e

$$
f_{(n+1) m}(v, w)=r g(v, w)-\sum_{i=1}^{(n+1) m} r\left\langle g(v, w), \nu_{i-1}\right\rangle_{X} \nu_{i-1}
$$

Observemos que para o problema contínuo temos um teorema análogo ao teorema 3.21 .

Teorema 3.22 Sejag de classe $C^{2}$, limitada com primeira e segunda derivadas limitadas. Então, para $m$ suficientemente grande, existe uma variedade invariante, suave e exponencialmente atratora, $S_{(n+1) m}$ para (3.11). O fluxo sobre $S_{(n+1) m}$ é dado por $U(t, x)=\left(v(t), \sigma_{(n+1) m}(v(t))\right)$, sendo $v$ solução de

$$
\dot{v}+B_{(n+1) m} v=h_{(n+1) m}\left(v, \sigma_{(n+1) m}(v)\right)
$$

Além disso, $s((n+1) m)=\left\|\sigma_{(n+1) m}\right\|=\sup _{v \in W}\left\|\sigma_{(n+1) m}(v)\right\|$ e a constante de Lipschitz, $l((n+1) m)$, de $\sigma_{(n+1) m}$ convergem para zero quando $m \rightarrow \infty$.

\section{Demonstração}

Iremos usar o teorema 3.20. Para isto vamos escrever $L_{h}=L_{f}=L_{g}$, sendo $L_{g}$ a constante de Lipschitz da função $g$ e $N_{h}=N_{f}=\|g\|_{\infty}$.

Observemos também que as constantes $\beta((n+1) m)$ e $\rho((n+1) m)$ são, neste caso, $\lambda_{(n+1) m}$ e $\lambda_{(n+1) m-1}$, respectivamente. Assim, temos que $\beta((n+1) m)-\rho((n+1) m)=\operatorname{ar} \pi^{2}(2(n+1) m-1)$. 
Mostramos, a seguir, a convergência uniforme dos autovalores e autovetores do problema discretizado para os autovalores e autofunções do problema contínuo, quando $m \rightarrow \infty$.

Teorema 3.23 Os $(n+1) m$ primeiros autovalores do operador $\tilde{B}_{(n+1) m}$ convergem uniformemente aos $(n+1) m$ primeiros autovalores do operador $B_{(n+1) m}$, quando $m \rightarrow \infty$.

\section{Demonstração}

Os $(n+1) m$ primeiros autovalores dos operadores $\tilde{B}_{(n+1) m}$ e $B_{(n+1) m}$ são, respectivamente, $\lambda_{k}^{m^{3}}=4 a r\left(m^{3}\right)^{2} \sin ^{2} \frac{k \pi}{2 m^{3}}+r$ e $\lambda_{k}=a r(k \pi)^{2}+r$, com $k=0, \ldots,(n+1) m-1$. Logo,

$$
\begin{aligned}
\left|\lambda_{k}^{m^{3}}-\lambda_{k}\right| & =\operatorname{ar}(k \pi)^{2}\left|\frac{\left(2 m^{3}\right)^{2}}{(k \pi)^{2}} \sin ^{2} \frac{k \pi}{2 m^{3}}-1\right| \\
& =\operatorname{ar}(k \pi)^{2}\left|\left(\frac{\sin k \pi / 2 m^{3}}{k \pi / 2 m^{3}}\right)^{2}-1\right|
\end{aligned}
$$

E usando a expansão em série de potência da função seno obtemos,

$$
\begin{aligned}
& \left|\lambda_{k}^{m^{3}}-\lambda_{k}\right| \\
& \quad=\operatorname{ar}(k \pi)^{2}\left|1-\frac{2}{3 !}\left(\frac{k \pi}{2 m^{3}}\right)^{2}+o\left(\left(\frac{k \pi}{2 m^{3}}\right)^{4}\right)-1\right| \\
& \leq \operatorname{ar}((n+1) m \pi)^{2}\left|\frac{2}{3 !}\left(\frac{(n+1) \pi}{2 m^{2}}\right)^{2}+o\left(\left(\frac{(n+1) \pi}{2 m^{2}}\right)^{4}\right)\right| \\
& =o\left(\frac{1}{m^{2}}\right) .
\end{aligned}
$$

Assim, $\left|\lambda_{k}^{m^{3}}-\lambda_{k}\right| \rightarrow 0$ uniformemente para todo $k=0, \ldots,(n+1) m$ quando $m \rightarrow \infty$.

Para concluir, mostramos a convergência uniforme das autofunções. 
Teorema 3.24 Os $(n+1) m$ primeiros autovetores do operador $\tilde{B}_{(n+1) m}$ convergem uniformemente às $(n+1) m$ primeiras autofunções do operador $B_{(n+1) m}$, quando $m \rightarrow \infty$.

\section{Demonstração}

Vamos mostrar que $\left\|\nu_{k}^{(n+1), m^{3}}(x)-\nu_{k}(x)\right\|_{\infty}<\varepsilon$ para $m$ suficientemente grande e para todo $k=0, \ldots,(n+1) m-1$.

Para isto, observemos que $\left|\cos k \pi x-\cos k \pi x_{j}\right| \leq \frac{k \pi}{m^{3}}$ se $x \in\left[\frac{j-1}{m^{3}}, \frac{j}{m^{3}}\right]$, o que implica $\left|\cos k \pi x-\cos k \pi x_{j}\right| \leq \frac{(n+1) \pi}{m^{2}}$, para $k=0, \ldots,(n+1) m-1$ e $j=1, \ldots, m^{3}$ e $\lim _{m \rightarrow \infty} \frac{1}{m^{3}} \sum_{j=1}^{m^{3}} \cos ^{2} k \pi x_{j}=\frac{1}{2}$.

Então,

$$
\begin{aligned}
\left\|\nu_{k}^{(n+1), m^{3}}(x)-\nu_{k}(x)\right\|_{\infty} & =\sup _{x \in[0,1]}\left|\nu_{k}^{(n+1), m^{3}}(x)-\nu_{k}(x)\right| \\
& =\sup _{x \in[0,1]} \sum_{i=0}^{n}\left|{ }_{i} \nu_{k}^{(n+1), m^{3}}(x)-{ }_{i} \nu_{k}(x)\right| \\
& \leq \frac{\sqrt{2}(n+1) \pi}{m^{2}} \sum_{i=0}^{n}\left|\frac{1}{N\left(\lambda_{k}^{m^{3}}\right)} \frac{n^{i}}{\left(n-\lambda_{k}^{m^{3}}\right)^{i}}\right|
\end{aligned}
$$

Vamos, a seguir, mostrar a proximidade dos campos vetoriais, o que nos garante a proximidade das soluções dos problemas contínuo e discretizado na variedade invariante e com isto temos a semi-continuidade superior dos atratores do problema discretizado, como nos afirma o teorema abaixo.

Teorema 3.25 Para cada $m>0$, os atratores do problema discretizado (3.6) são semi-contínuos superiormente em $m=\infty$. 


\section{Demonstração}

Mostramos, a princípio, a proximidade dos campos vetoriais e para isto vamos denotar por $\tilde{h}_{(n+1) m}^{i}$ e $h_{(n+1) m}^{i}$ a $i$-ésima função coordenada de $\tilde{h}_{(n+1) m}$ e $h_{(n+1) m}$, respectivamente.

Assim temos,

$$
\begin{aligned}
& \left|\tilde{h}_{(n+1) m}^{i}(v, 0)-h_{(n+1) m}^{i}(v, 0)\right| \\
& =\mid \frac{r}{m^{3}} \sum_{j=1}^{m^{3}} g\left(\sum_{k=1}^{(n+1) m} \frac{v_{k}\left[\mu_{k-1}^{m^{3}}\right]_{j}}{N\left(\lambda_{k-1}^{m^{3}}\right)}, \sum_{k=1}^{(n+1) m} \frac{v_{k} \frac{n^{n}}{\left(n-\lambda_{k-1}^{m^{3}}\right)^{n}}\left[\mu_{k-1}^{m^{3}}\right]_{j}}{N\left(\lambda_{k-1}^{m^{3}}\right)}\right) \frac{\left[\mu_{i-1}^{m^{3}}\right]_{j}}{N\left(\lambda_{i-1}^{m^{3}}\right)} \\
& -r \int_{0}^{1} g\left(\sum_{k=1}^{(n+1) m} \frac{v_{k} \mu_{k-1}(x)}{N\left(\lambda_{k-1}^{m^{3}}\right)}, \sum_{k=1}^{(n+1) m} \frac{v_{k} \frac{n^{n}}{\left(n-\lambda_{k-1}^{m^{3}}\right)^{n}} \mu_{k-1}(x)}{N\left(\lambda_{k-1}^{m^{3}}\right)}\right) \frac{\mu_{i-1}(x)}{N\left(\lambda_{i-1}^{m^{3}}\right)} d x \mid \\
& =r \mid \sum_{j=1}^{m^{3}} \int_{\frac{j-1}{m^{3}}}^{\frac{j}{m^{3}}}\left\{g\left(\sum_{k=1}^{(n+1) m} \frac{v_{k}\left[\mu_{k-1}^{m^{3}}\right]_{j}}{N\left(\lambda_{k-1}^{m^{3}}\right)}, \sum_{k=1}^{(n+1) m} \frac{v_{k} \frac{n^{n}}{\left(n-\lambda_{k-1}^{m^{3}}\right)^{n}}\left[\mu_{k-1}^{m^{3}}\right]_{j}}{N\left(\lambda_{k-1}^{m^{3}}\right)}\right) \frac{\left[\mu_{i-1}^{m^{3}}\right]_{j}}{N\left(\lambda_{i-1}^{m^{3}}\right)}\right. \\
& \left.-g\left(\sum_{k=1}^{(n+1) m} \frac{v_{k} \mu_{k-1}(x)}{N\left(\lambda_{k-1}^{m^{3}}\right)}, \sum_{k=1}^{(n+1) m} \frac{v_{k} \frac{n^{n}}{\left(n-\lambda_{k-1}^{m^{3}}\right)^{n}} \mu_{k-1}(x)}{N\left(\lambda_{k-1}^{m^{3}}\right)}\right) \frac{\mu_{i-1}(x)}{N\left(\lambda_{i-1}^{m^{3}}\right)}\right\} d x \mid \\
& \leq r \mid \sum_{j=1}^{m^{3}} \int_{\frac{j-1}{m^{3}}}^{\frac{j}{m^{3}}} g\left(\sum_{k=1}^{(n+1) m} \frac{v_{k}\left[\mu_{k-1}^{m^{3}}\right]_{j}}{N\left(\lambda_{k-1}^{m^{3}}\right)}, \sum_{k=1}^{(n+1) m} \frac{v_{k} \frac{n^{n}}{\left(n-\lambda_{k-1}^{m^{3}}\right)^{n}}\left[\mu_{k-1}^{m^{3}}\right]_{j}}{N\left(\lambda_{k-1}^{m^{3}}\right)}\right) \\
& \text { - }\left(\frac{\left[\mu_{i-1}^{m^{3}}\right]_{j}}{N\left(\lambda_{i-1}^{m^{3}}\right)}-\frac{\mu_{i-1}(x)}{N\left(\lambda_{i-1}^{m^{3}}\right)}\right) d x \mid \\
& +r \mid \sum_{j=1}^{m^{3}} \int_{\frac{i-1}{m^{3}}}^{\frac{j}{m^{3}}}\left\{g\left(\sum_{k=1}^{(n+1) m} \frac{v_{k}\left[\mu_{k-1}^{m^{3}}\right]_{j}}{N\left(\lambda_{k-1}^{m^{3}}\right)}, \sum_{k=1}^{(n+1) m} \frac{v_{k} \frac{n^{n}}{\left(n-\lambda_{k-1}^{m^{3}}\right)^{n}}\left[\mu_{k-1}^{m^{3}}\right]_{j}}{N\left(\lambda_{k-1}^{m^{3}}\right)}\right)\right. \\
& \left.-g\left(\sum_{k=1}^{(n+1) m} \frac{v_{k} \mu_{k-1}(x)}{N\left(\lambda_{k-1}^{m^{3}}\right)}, \sum_{k=1}^{(n+1) m} \frac{v_{k} \frac{n^{n}}{\left(n-\lambda_{k-1}^{m^{3}}\right)^{n}}\left[\mu_{k-1}^{m^{3}}\right]_{j}}{N\left(\lambda_{k-1}^{m^{3}}\right)}\right)\right\} \frac{\mu_{i-1}(x)}{N\left(\lambda_{i-1}^{m^{3}}\right)} d x \mid \\
& +r \mid \sum_{j=1}^{m^{3}} \int_{\frac{j-1}{m^{3}}}^{\frac{j}{m^{3}}}\left\{g\left(\sum_{k=1}^{(n+1) m} \frac{v_{k} \mu_{k-1}(x)}{N\left(\lambda_{k-1}^{m^{3}}\right)}, \sum_{k=1}^{(n+1) m} \frac{v_{k} \frac{n^{n}}{\left(n-\lambda_{k-1}^{m^{3}}\right)^{n}}\left[\mu_{k-1}^{m^{3}}\right]_{j}}{N\left(\lambda_{k-1}^{m^{3}}\right)}\right)\right. \\
& \left.-g\left(\sum_{k=1}^{(n+1) m} \frac{v_{k} \mu_{k-1}(x)}{N\left(\lambda_{k-1}^{m^{3}}\right)}, \sum_{k=1}^{(n+1) m} \frac{v_{k} \frac{n^{n}}{\left(n-\lambda_{k-1}^{m^{3}}\right)^{n}} \mu_{k-1}(x)}{N\left(\lambda_{k-1}^{m^{3}}\right)}\right)\right\} \frac{\mu_{i-1}(x)}{N\left(\lambda_{i-1}^{m^{3}}\right)} d x \mid \\
& \leq r \int_{0}^{1}\|g\|_{\infty} \frac{\sqrt{2}(n+1) \pi}{m^{2}} d x
\end{aligned}
$$




$$
\begin{aligned}
& +r \sum_{j=1}^{m^{3}} \int_{\frac{j-1}{m^{3}}}^{\frac{j}{m^{3}}} L_{g}\left|\sum_{k=1}^{(n+1) m} \frac{v_{k}\left[\mu_{k-1}^{m^{3}}\right]_{j}}{N\left(\lambda_{k-1}^{m^{3}}\right)}-\sum_{k=1}^{(n+1) m} \frac{v_{k} \mu_{k-1}(x)}{N\left(\lambda_{k-1}^{m^{3}}\right)}\right|\left|\frac{\mu_{i-1}(x)}{N\left(\lambda_{i-1}^{m^{3}}\right)}\right| d x \\
& +r \sum_{j=1}^{m^{3}} \int_{\frac{j-1}{m^{3}}}^{\frac{j}{m^{3}}} L_{g}\left|\sum_{k=1}^{(n+1) m} \frac{v_{k} \frac{n^{n}}{\left(n-\lambda_{k-1}^{m}\right)^{n}}\left[\mu_{k-1}^{m^{3}}\right]_{j}}{N\left(\lambda_{k-1}^{m^{3}}\right)}-\sum_{k=1}^{(n+1) m} \frac{v_{k} \frac{n^{n}}{\left(n-\lambda_{k-1}^{m^{3}}\right)^{n}} \mu_{k-1}(x)}{N\left(\lambda_{k-1}^{m^{3}}\right)}\right| \\
& \text { - }\left|\frac{\mu_{i-1}(x)}{N\left(\lambda_{i-1}^{m^{3}}\right)}\right| d x \\
& \leq r \int_{0}^{1}\|g\|_{\infty} \frac{\sqrt{2}(n+1) \pi}{m^{2}} d x \\
& +r \sum_{j=1}^{m^{3}} \int_{\frac{i-1}{m^{3}}}^{\frac{j}{m^{3}}} L_{g} \sum_{k=1}^{(n+1) m}\left|\frac{v_{k}}{N\left(\lambda_{k-1}^{m^{3}}\right)} \frac{\sqrt{2}(n+1) \pi}{m^{2}}\right|\left|\frac{\mu_{i-1}(x)}{N\left(\lambda_{i-1}^{m^{3}}\right)}\right| d x \\
& +r \sum_{j=1}^{m^{3}} \int_{\frac{j-1}{m^{3}}}^{\frac{j}{m^{3}}} L_{g} \sum_{k=1}^{(n+1) m}\left|\frac{v_{k} \frac{n^{n}}{\left(n-\lambda_{k-1}^{m^{3}}\right)^{n}}}{N\left(\lambda_{k-1}^{m^{3}}\right)} \frac{\sqrt{2}(n+1) \pi}{m^{2}}\right|\left|\frac{\mu_{i-1}(x)}{N\left(\lambda_{i-1}^{m^{3}}\right)}\right| d x \\
& \leq r\|g\|_{\infty} \frac{\sqrt{2}(n+1) \pi}{m^{2}} \\
& +r \int_{0}^{1} L_{g} \frac{2(n+1) \pi}{m^{2}} \sum_{k=1}^{(n+1) m}\left|\frac{v_{k}}{N\left(\lambda_{k-1}^{m^{3}}\right)}\right| \frac{1}{N\left(\lambda_{i-1}^{m^{3}}\right)} d x \\
& +r \int_{0}^{1} L_{g} \frac{2(n+1) \pi}{m^{2}} \sum_{k=1}^{(n+1) m}\left|\frac{v_{k} \frac{n^{n}}{\left(n-\lambda_{k-1}^{m^{3}}\right)^{n}}}{N\left(\lambda_{k-1}^{m^{3}}\right)}\right| \frac{1}{N\left(\lambda_{i-1}^{m^{3}}\right)} d x \\
& =r\|g\|_{\infty} \frac{\sqrt{2}(n+1) \pi}{m^{2}} \\
& +r L_{g} \frac{2(n+1) \pi}{m^{2}} \frac{1}{N\left(\lambda_{i-1}^{m^{3}}\right)} \sum_{k=1}^{(n+1) m}\left|\frac{v_{k}}{N\left(\lambda_{k-1}^{m^{3}}\right)}\right| \\
& +r L_{g} \frac{2(n+1) \pi}{m^{2}} \frac{1}{N\left(\lambda_{i-1}^{m^{3}}\right)} \sum_{k=1}^{(n+1) m}\left|\frac{v_{k} \frac{n^{n}}{\left(n-\lambda_{k-1}^{m^{3}}\right)^{n}}}{N\left(\lambda_{k-1}^{m^{3}}\right)}\right|
\end{aligned}
$$

e assim obtemos a proximidade dos campos vetoriais seguindo o trabalho de Bruschi, Carvalho e Ruas-Filho [2].

Com isto temos a proximidade das soluções dos problemas contínuo e discretizado na variedade invariante.

Consideremos agora $\mathcal{A}_{(n+1) m} \subset \mathbb{R}^{(n+1) m^{3}}$ os atratores do problema discretizado (3.6) e $\mathcal{A} \subset\left(L^{2}(0,1)\right)^{(n+1)}$ o atrator do problema contínuo (3.2). E a fim de obtermos a imersão de $\mathbb{R}^{(n+1) m^{3}}$ em $\left(L^{2}(0,1)\right)^{(n+1)}$ consideremos 
$U=\left(U_{0}^{1}, \ldots, U_{0}^{m^{3}}, U_{1}^{1}, \ldots, U_{1}^{m^{3}}, \ldots, U_{n}^{1}, \ldots, U_{n}^{m^{3}}\right)^{T} \in \mathbb{R}^{(n+1) m^{3}}$ e definimos a inclusão

$$
\begin{aligned}
i: \mathbb{R}^{(n+1) m^{3}} & \rightarrow\left(L^{2}(0,1)\right)^{(n+1)} \\
U & \mapsto U(x)=\left(\sum_{j=1}^{m^{3}} U_{0}^{j} \mathcal{X}_{\left[0, \frac{1}{m^{3}}\right]}(x), \ldots, \sum_{j=1}^{m^{3}} U_{n}^{j} \mathcal{X}_{\left[\frac{m^{3}-1}{m^{3}}, 1\right]}(x)\right)
\end{aligned}
$$

Assim, temos que dado $\varepsilon>0$ existe $m_{0}>0$ tal que $i\left(\mathcal{A}_{(n+1) m}\right) \subset \mathcal{A}^{\varepsilon}$ para todo $m>m_{0}$ e portanto $\operatorname{dist}\left(i\left(\mathcal{A}_{(n+1) m}\right), \mathcal{A}\right) \rightarrow 0$ quando $m \rightarrow \infty$. 


\section{Referências Bibliográficas}

[1] BOYCE, W.E., DIPRIMA, R.C. Equações diferenciais elementares e problemas de valores de contorno. Rio de Janeiro: Guanabara, 1977.

[2] BRUSCHI, S.M., CARVALHO, A.N., RUAS-FILHO, J.G. The dynamics of a one-dimensional parabolic problem versus the dynamics of its discretization. J. Diff. Eq., v.168, n.1, p.67-92, 2000.

[3] BUSENBERG, S., HUANG, W. Stability and Hopf bifurcation for a population delay model with diffusion effects. J. Diff. Eq., v.124, p.80-107, 1996.

[4] FARGUE, M.D. Réductibilité dês systèmes héréditaires à dês systèmes dynamiques: régis par des équations différentielles ou aux dérivées partielles. C. R. Acad. Sc. Paris, SerB, v.277, p.471-473, 1973.

[5] FIGUEIREDO, D.G. Análise de fourier e equações diferenciais parciais. Rio de Janeiro: Instituto de Matemática Pura e Aplicada, 1977. (Projeto Euclides).

[6] FRIESECKE, G. Exponentially growing solutions for a delay-diffusion equation with negative feedback. J. Diff. Eq., v.98, p.1-18, 1992.

[7] GEDEON, T., HINES, G. Upper semicontinuity of morse sets of a discretization of a delay-differential equation. $J$. Diff. Eq., v.151, n.1, p.36-78, 1999.

[8] HALE, J.K. Asymptotic behavior of dissipative systems. Providence: American Mathematical Society, 1988. (Mathematical Surveys and Monographs, 25).

[9] HALE, J.K., KATO, J. Phase space for retarded equations with infinite delay. Funk. Ekv., v.21, p.11-41, 1978.

[10] HENRÍQUEZ, H.R. Periodic solutions of quasi-linear partial functional differential equations with unbounded delay. Funk. Ekv., v.37, p.329-343, 1994.

[11] HENRÍQUEZ, H.R. Approximation of abstract functional differential equations with unbounded delay. Indian J. Pure Appl. Math., v.27, n.4, p.357-386, 1996. 
[12] HENRÍQUEZ, H.R. Regularity of solutions of abstract retarded functional differential equations witn unbounded delay. Nonlin. Anal., v.28, n.3, p.513-531, 1997.

[13] HENRY, D. Geometric theory of semilinear parabolic equations. New York: Springer-Verlag, 1981. (Lecture Notes in Mathematics, 840).

[14] HIGHAM, D.J., SARDAR, T.K. Existence and stability of fixed points for a discretised nonlinear reaction-diffusion equation with delay. Appl. Numer. Math., v.18, p.155-173, 1995.

[15] HINES, G. Convergence of continuous delays to discrete delays. In:U.S. Japan Conference on singular pertubations and dynamics, $\mathrm{Pi}-$ ne Mountains, 1996.

[16] HINES, G. Upper semicontinuity of the attractor with respect to parameter dependent delays. J. Diff. Eq., v.123, p.56-92, 1995.

[17] HINO, Y.H., MURAKAMI, S., NAITO, T. Functional differential equations with infinite delay. New York: Springer-Verlag, 1991. (Lecture Notes in Mathematics, 1473).

[18] HUTCHINSON, G.H. Circular causal systems in ecology. Ann. N. Y. Acad. Sci., v.50, p.221-246, 1948.

[19] LUCKHAUS, S. Global boundedness for a delay differential equation. Trans. Amer. Math. Soc., v.294, n.2, p.767-774, 1986.

[20] MOOD, A.M., GRAYBILL, F.A., BOES, D.C. Introduction to the theory of statistics. Tokio: McGraw-Hill, 1974. (McGraw-Hill Series in Probability and Statistics).

[21] MURRAY, J.D. Mathematical biology. New York: Springer-Verlag, 1989.

[22] OLIVA, S.M., CÒNSUL, N. Synchronization in herbivorous population models with diffusion and delays. Fields Inst. Commun., v.31, p.75-95, 2002 .

$$
\text { QA338.c c748d }
$$

[23] OLIVEIRA, L.A.F. Instability of homogeneous periodic solutions of parabolic-delay equations. J. Diff. Eq., v.109, n.1, p.42-76, 1994.

[24] PAZY, A. Semigroups of linear operators and applications to partial differential equations. New York: Springer-Verlag, 1983. (Applied Mathematical Sciences, 44).

[25] RUAN, S., WU, J. Reaction-diffusion equations with infinite delay. Canad. Appl. Math. Quart., v.2, n.4, p.485-550, 1994. 
[26] SARDAR, T.K., HIGHAM, D.J. Dynamics of constant and variable stepsize methods for a nonlinear population model with delay. Appl. Numer. Math., v.24, p.425-438, 1997.

[27] TRAVIS, C.C., WEBB, G.F. Existence and stability for partial functional differential equations. Trans. Amer. Math. Soc., v.200, p.395-418, 1974.

[28] TRAVIS, C.C., WEBB, G.F. Existence, stability, and compactness in the $\alpha$-norm for partial functional differential equations. Trans. Amer. Math. Soc., v.240, p.129-143, 1978.

[29] TURYN, L. A partial functional differential equation. J. Math. Anal. Appl., v.263, p.1-13, 2001.

[30] WENDI, W., ZHIEN, M. Asymptotic behavior of a predator-prey system with diffusion and delays. J. Math. Anal. Appl., v.206, p.191204, 1997.

[31] WU, J. Theory and applications of partial functional differential equations. New York: Springer-Verlag, 1996. (Applied Mathematical Sciences, 119). 